\title{
Star formation in M 33: Spitzer photometry of discrete sources ${ }^{\star}$
}

\author{
S. Verley ${ }^{1}$, L. K. Hunt ${ }^{2}$, E. Corbelli ${ }^{1}$, and C. Giovanardi ${ }^{1}$ \\ 1 Osservatorio Astrofisico di Arcetri, Largo E. Fermi 5, 50125 Firenze, Italy \\ e-mail: [simon; edvige; giova] @arcetri.astro.it \\ 2 INAF - Istituto di Radioastronomia-Sezione Firenze, Largo E. Fermi 5, 50125 Firenze, Italy \\ e-mail: hunt@arcetri.astro.it
}

Received 28 June 2007 / Accepted 12 September 2007

\section{ABSTRACT}

\begin{abstract}
Aims. Combining the relative vicinity of the Local Group spiral galaxy M 33 with the Spitzer images, we investigate the properties of infrared (IR) emission sites and assess the reliability of the IR emission as a star formation tracer.

Methods. The mid- and far-IR emission of M 33 was obtained from IRAC and MIPS images from the Spitzer archive. We compared the photometric results for several samples of three known types of discrete sources (HII regions, supernovae remnants and planetary nebulae) with theoretical diagnostic diagrams, and derived the spectral energy distribution (from 3.6 to $24 \mu \mathrm{m}$ ) of each type of object. Moreover, we generated a catalogue of $24 \mu \mathrm{m}$ sources and inferred their nature from the observed and theoretical colours of the known type sources. We estimated the star formation rate in M33 both globally and locally, from the IR emission and from the H $\alpha$ emission line.

Results. The colours of the typical IR emissions of HII regions, supernovae remnants and planetary nebulae are continuous among the different samples, with overlapping regions in the diagnostic diagrams. The comparison between the model results and the colours of HII regions indicates a dusty envelope at relatively high temperatures $\sim 600 \mathrm{~K}$, and moderate extinction $A_{V} \lesssim 10$. The $24 \mu \mathrm{m}$ sources IR colours follow the regions observationally defined by the three classes of known objects but the majority of them represent HII regions. The derived total IR luminosity function is in fact very similar to the HII luminosity function observed in the Milky Way and in other late type spirals. Even though our completeness limit is $5 \times 10^{37} \mathrm{erg} \mathrm{s}^{-1}$, in low density regions we are able to detect sources five times fainter than this, corresponding to the faintest possible HII region. The 8 and $24 \mu \mathrm{m}$ luminosities within the central $5 \mathrm{kpc}$ of M 33 are comparable and of order $4 \times 10^{28} \mathrm{erg} \mathrm{s}^{-1} \mathrm{~Hz}^{-1}\left(v L_{v}(8)=1.5 \times 10^{42}\right.$ and $\left.v L_{v}(24)=4.4 \times 10^{41} \mathrm{erg} \mathrm{s}^{-1}\right)$. We estimate the total IR emission in the same region of M 33 to be $10^{9} L_{\odot}$. The discrete sources account for about one third of the $24 \mu \mathrm{m}$ emission while the rest is diffuse. From the IR emission, we derive a star formation rate for the inner disk equal to $0.2 M_{\odot} \mathrm{yr}^{-1}$, consistent with the star formation rate obtained from the $\mathrm{H} \alpha$ emission.
\end{abstract}

Key words. galaxies: individual: M 33 - galaxies: ISM - galaxies: Local Group - galaxies: spiral

\section{Introduction}

The interstellar medium (ISM) in galaxies provides the raw material from which stars form, and is the repository of the products of stellar evolution. Stars form in condensations of cool molecular gas, eventually destroying their birth sites through energetic photons, massive stellar winds, and supernova (SN) explosions. Dust is formed through coagulation in stellar winds and supernova ejecta, and over time, becomes an integral part of the ISM. Radiative and mechanical energy are input to the ISM through massive star formation and evolution, in the form of ionising photons, supernova explosions, and magnetic fields. The ISM properties, such as magnetic field, gas, and dust, affect star formation processes on all scales. At the same time, star formation drives the evolution of the ISM. On the long term, local processes of star formation can influence large-scale structure in the ISM and determine how galaxies evolve. Ultraviolet and optical wavelengths are not the best wavelengths for SF studies, because dust can hide massive star formation, mask the effects of feedback, and redistribute the ISM energy and radiation. However, first IRAS (Neugebauer et al. 1984), then ISO (Kessler et al. 1996), and now Spitzer (Werner et al. 2004) have been able

$\star$ Tables 2 and 3 are only available in electronic form at http://www . aanda. org to penetrate the dust in the ISM, and thus better study the interaction among its constituents.

In this series of papers, we examine star formation properties of the Local Group late type spiral M33. The proximity of Local Group members together with Spitzer resolution makes possible the resolution of structures on spatial scales of 5-10 pc, and enable us to effectively study individual star forming sites and their surrounding environment in a galaxy different from our own. M 33, is at a distance of $840 \mathrm{kpc}$ (Freedman et al. 1991), its mass and apparent size are smaller than that of M 31 but M 33 hosts the brightest HII complex in the Local Group. This, together with its blue colour assures us that star formation is still very active throughout its disk. M33 bears no signs of recent mergers and therefore gives us a unique opportunity for investigating the interplay of gas, dust, and star formation in isolated galaxy disks. This is possible thanks also to high resolution and sensitive observations now becoming available for this nearby galaxy at all wavelengths. Chemical abundances have been measured in stellar populations of different ages (e.g. Magrini et al. 2007a,b), and surveys of atomic and molecular hydrogen point out the location of massive gas clumps (Engargiola et al. 2003; Heyer et al. 2004). Through detailed dynamical mass modeling of the galaxy we can now trace the mass distribution of visible and dark matter (Corbelli \& Salucci 2000; Corbelli 2003). 
Our focus here is first on M33's population of discrete known type of point sources: HII regions, supernova remnants (SNRs), and planetary nebulae (PNe). In principle, these sources track different phases of the star formation process, from the generation of ionising photons and stellar winds in massive stars, to subsequent explosion as $\mathrm{SNe}$ and finally to the more evolved stage of PNe. Hence, our immediate goal is to assess the physical conditions of the stellar environment at different stages. The second purpose of this paper is the generation of an IR source catalogue from the Spitzer MIPS observations at $24 \mu \mathrm{m}$. Here we shall use IRAC-MIPS diagnostic diagrams of known sources to better understand the nature of these sources. In a subsequent paper we will relate the IR properties with detailed observations of the surrounding ISM at other wavelengths and to the disk large-scale structure. Spitzer data and the vast multi-wavelength dataset available for M 33 will help understand how star formation proceeds in low luminosity spiral galaxies and how this relates to other global galaxy properties.

The present paper is structured as follows: in Sect. 2, we present the Spitzer IRAC and MIPS data and their reduction process. The large scale structure of the dust emission in M33 is investigated through colour images in Sect. 3. We analyse the IRAC and MIPS 24 photometry of discrete type-known sources (HII regions, PNe, SNRs) in Sect. 4 and compare their colours to theoretical diagnostic diagrams in Sect. 5. In Sect. 6, we present a catalogue of the sources emitting in the $24 \mu \mathrm{m}$ MIPS band and interpret their nature in light of the results obtained in the previous Section. The reliability of the IR emission as a star formation tracer, through comparison with $\mathrm{H} \alpha$, is investigated in Sect. 7. Finally, the summary and conclusions of our study are given in Sect. 8.

\section{Observations and data reduction}

We retrieved images from the Guaranteed Time Observations (PID 5, PI R. Gehrz) in the Spitzer Space Telescope (Werner et al. 2004) data archive. The Spitzer Space Telescope carries two photometric cameras: the Infrared Array Camera (IRAC) and the Multiband Imaging Photometer (MIPS).

\subsection{IRAC data}

The Infrared Array Camera (Fazio et al. 2004) is equipped with two detector arrays of $256 \times 256$ pixels. The field of view of a single array is $5.2 \times 5.2$. We analyze here eight sets of IRAC observations of M 33 (AORs 3636224, 3636480, 3637760, 3638016, 3638784, 3639040, 3640320, 3640576) in all four IRAC bands. The Basic Calibrated Data (BCD) were created by the Spitzer Science Center (SSC) pipeline, version S14.0.0 for all AORs. As the first data frame of each observation sequence has a shorter integration time than the regular exposure time (10.4 s), we discarded those frames. A more complete description of the observations is given by Hinz et al. (2004), McQuinn et al. (2007), and Tabatabaei et al. (2007a).

The mosaics were assembled gathering all the BCDs for each wavelength. The individual calibrated frames were processed using Mopex (Makovoz \& Khan 2005), with a cosmic-ray rejection and a background matching applied between overlapping fields of view. During the reduction, we used a common value of the pixel size equal to 1 '.20. The final number of individual BCDs used is about 1700 , in each IRAC channel. The final dimension of the mosaics is approximately $62 ! 4 \times 91: 7$. Because of the overlap between adjacent BCDs, the mean redundancy is 12 (from 6 to 30 ).
The obtained IRAC mosaics were background subtracted, estimating 20 sky levels (the median values in $5 \times 5$ pixels boxes) near the edges of the mosaics, the farthest from the galaxy centre. The 5.8 and $8.0 \mu \mathrm{m}$ channels presented a gradient in the background, and we removed it using a fitted surface with the IRAF task imsurfit (images.imfit). In each channel, the final background value subtracted is the mean of the 20 median values: 0.059 at $3.6 \mu \mathrm{m}, 0.146$ at $4.5 \mu \mathrm{m}, 1.475$ at $5.8 \mu \mathrm{m}$, and 5.389 at $8.0 \mu \mathrm{m}$. The mosaics were then aligned using the IRAF tasks geomap and geotran (in immatch) using 15 point sources.

Because of the diffraction limit of the telescope, the images have a resolution of 1. .7 to 2 '.0 (see IRAC Observing Manual) which translate into 6.7 to $8 \mathrm{pc}$ at the distance of M 33. The final IRAC mosaics at 3.6, 4.5, 5.8, and $8.0 \mu \mathrm{m}$ are shown in Fig. 1.

\subsection{MIPS data}

Images of M 33 at wavelengths 24, 70, and $160 \mu \mathrm{m}$ (with bandwidths of 5,19 , and $35 \mu \mathrm{m}$, respectively) were obtained using data collected by the Multiband Imaging Photometer (Rieke et al. 2004). MIPS uses true detector arrays: $128 \times 128$ pixels at $24 \mu \mathrm{m}$ (Si:As detector), $32 \times 32$ pixels at $70 \mu \mathrm{m}$ (Ge:Ga device) and $2 \times 20$ pixels at $160 \mu \mathrm{m}$ (Ge:Ga device). The nominal fields of view are $5^{\prime} \times 5^{\prime}$ at 24 and $70 \mu \mathrm{m}$ and $0^{\prime} .75 \times 5^{\prime}$ at $160 \mu \mathrm{m}$. The resolution of the instrument is diffraction limited to $6^{\prime \prime}, 18^{\prime \prime}$, and $40^{\prime \prime}$ at 24,70 , and $160 \mu \mathrm{m}$, respectively. The operating modes of all the observations were "scan map", the telescope scanning the sky at a constant rate.

As for IRAC, we retrieved eight MIPS datasets from the archive (AORs 3647744, 3648000, 3648256, 3648512, 3648768, 3649024,3649280 and 3649536). The $24 \mu \mathrm{m}$ BCDs were created by the SSC pipeline version S16.1.0. The exposure times of the DCEs were $3.67 \mathrm{~s}$ (except for the first frames of each series, where the integration time per pixel is lower and which consequently were not used in the further reduction steps). Since the observations were acquired over two epochs, the respective mosaics were assembled separately using Mopex. For consistence with the IRAC reduction, the pixel size was chosen to be 1 '.20. The first epoch includes 4199 frames, and the second one includes 4500 frames. This latter map is displayed in Fig. 2. The mean redundancy per sky position is 22 (varying from 10 to 40), in each mosaic. This high level of redundancy removes any spurious effects such as cosmic-ray hits and provides more reliable measurements. In the present article, as we plan to focus on point sources (HII regions, SNRs and PNe) located near the centre of the galaxy, we chose to concentrate, for each epoch, on the two central AORs. These two images were then aligned with the reference of the coordinates of 8 point sources and combined together by keeping the average of the pixel values. A small background gradient was still visible in the combined image and was removed by fitting a surface to selected regions (IRAF task: imsurfit). The zone defined by the superposition of the two central AORs of each epoch is marked on the $24 \mu \mathrm{m}$ image (see Fig. 2). The resolution measured on the final image is $6^{\prime \prime}$.

The 70 and $160 \mu \mathrm{m}$ MIPS observations were acquired with the same AORs as the $24 \mu \mathrm{m}$ observations. The version S16.1.0 of the SSC pipeline was also used to create the BCDs. The integration times of the DCEs is $4.19 \mathrm{~s}$, both for the 70 and $160 \mu \mathrm{m}$ observations. We used Mopex to create the final mosaics (again the pixel sizes were chosen to be 1 '. 2 ), including 8750 individual frames at each of the two wavelength channels. The mean redundancy is about 20 (from 8 to 42) and 6 (from 2 to 10) at 70 and $160 \mu \mathrm{m}$, respectively. The final images show some striping along the scan direction that is a residual instrumental artifacts due to 

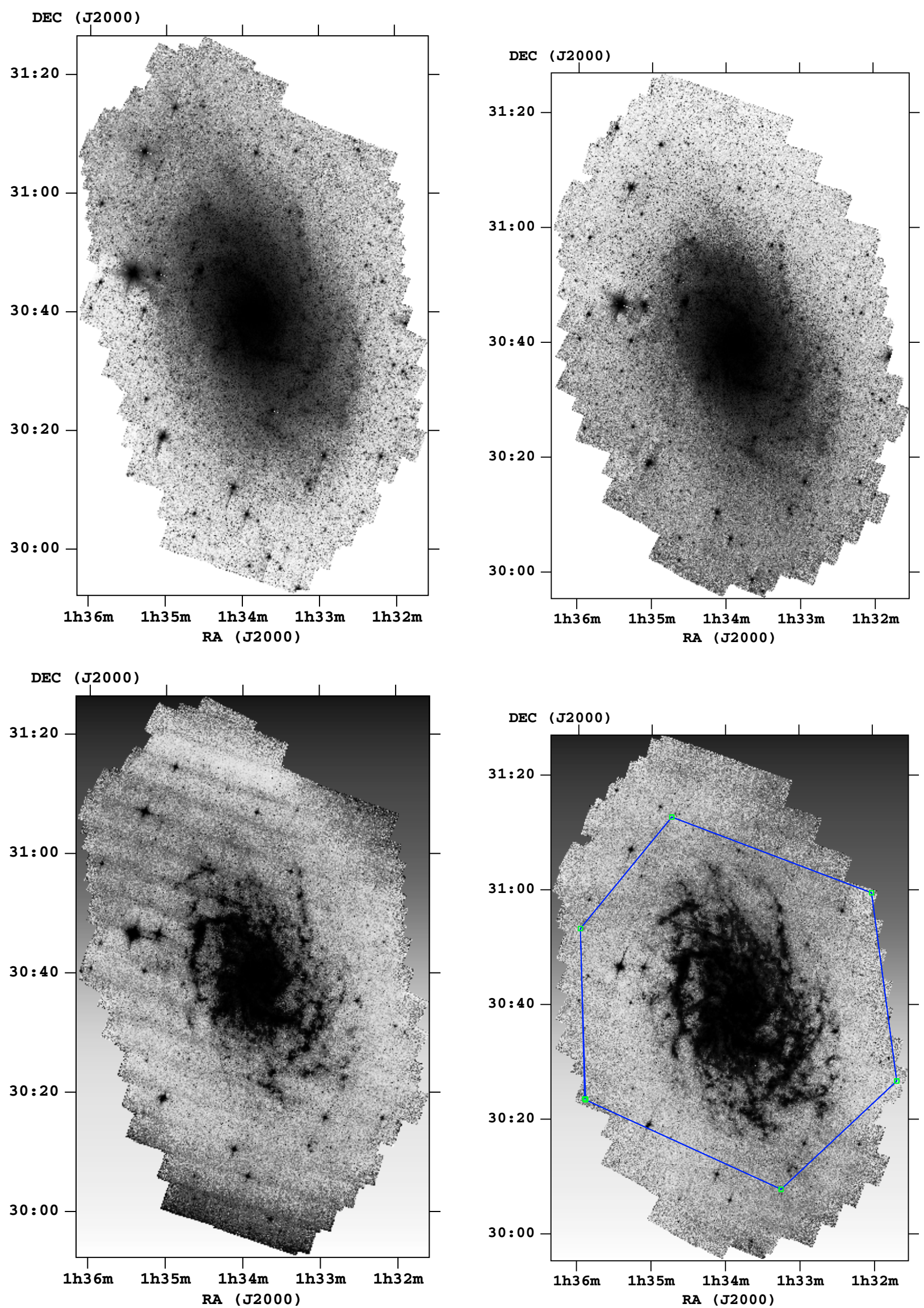

Fig. 1. Final IRAC mosaics of M 33. The upper left image displays the $3.6 \mu \mathrm{m}$, the upper right $4.5 \mu \mathrm{m}$, the lower left $5.8 \mu \mathrm{m}$ and the lower right the $8.0 \mu \mathrm{m}$ channel. The images are about $1^{\circ}$ by $1.5^{\circ}$, north is up and east is to the left. The region enclosed by the continuous line will be further investigated in the present paper. 

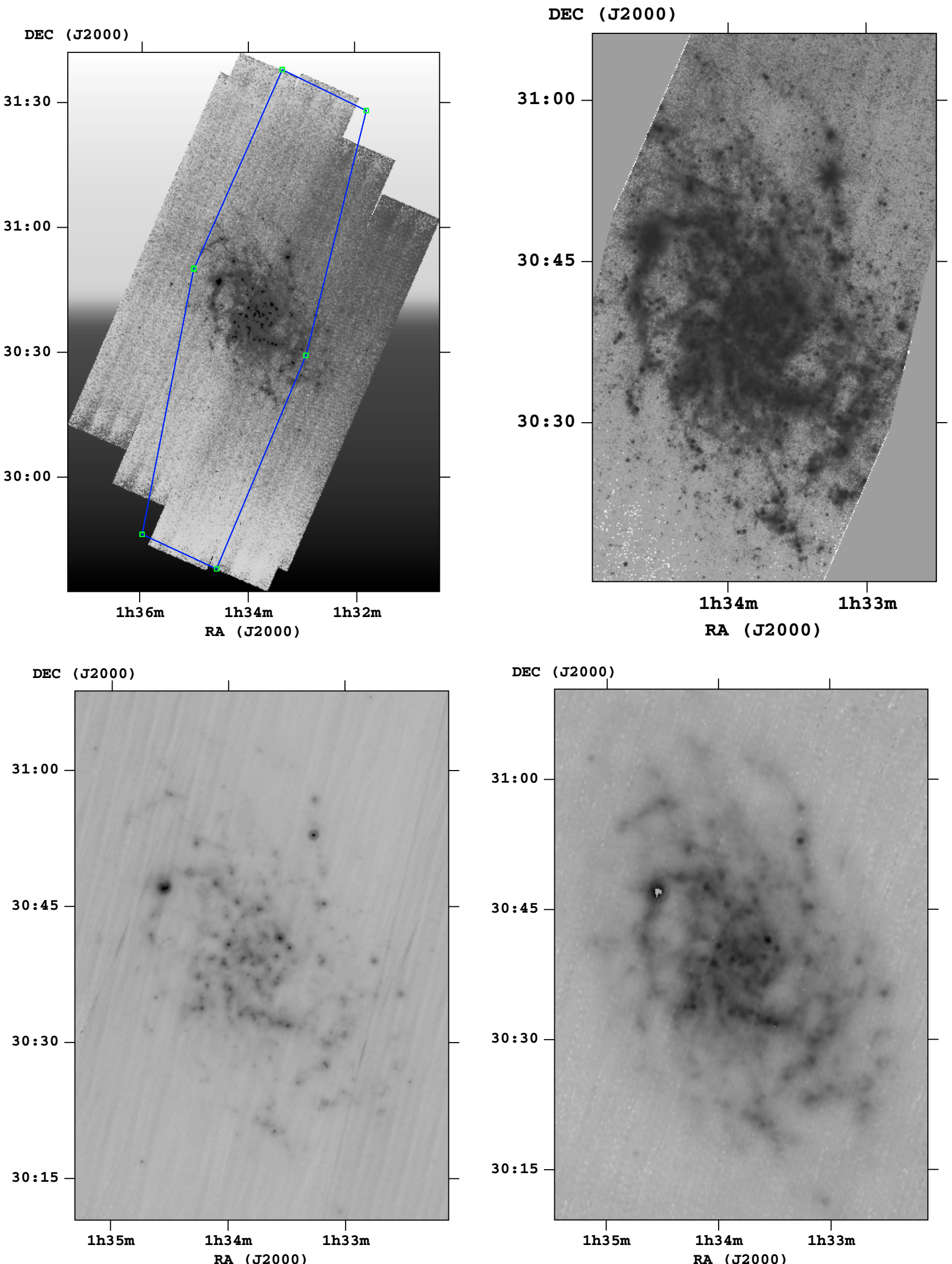

Fig. 2. MIPS images of M33. The upper panels display the $24 \mu \mathrm{m}$ images: the full image (one single epoch) is on the left. The inner region enclosed by the continuous line will be further investigated in the present paper, and is shown in the upper right panel after overlapping the data from two different epochs. The bottom left panel shows the $70 \mu \mathrm{m}$ channel, and the lower right the $160 \mu \mathrm{m}$ channel. The images are about $30^{\prime} \times$ $50^{\prime}$, north is up and east is left. 
the time-dependent responsivity of the Ge:Ga detectors (Helou et al. 2004; Hinz et al. 2004). The final resolution is about $16^{\prime \prime}$ and $40^{\prime \prime}$ for the 70 and $160 \mu \mathrm{m}$ images, respectively. The final MIPS images $(24,70$, and $160 \mu \mathrm{m})$ are shown in Fig. 2.

\section{3. $H \alpha$ image}

We also include in our analysis an optical narrow-band $\mathrm{H} \alpha$ image, a standard star formation indicator. The M $33 \mathrm{H} \alpha$ emission was observed at the Kitt Peak National Observatory (NOAO). The dimensions of the CCD are $2048 \times 2048$ with pixels of 2'.03 and a total field of view of about $70^{\prime} \times 70^{\prime}$. More details of the observations and reduction process can be found in Hoopes \& Walterbos (2000); the estimated [N II] contamination accounts for at most $5 \%$ of the measured flux. The total $\mathrm{H} \alpha$ luminosity of M33 is about $7 \times 10^{6} L_{\odot}$ and is dominated by HII regions (Devereux et al. 1997). The optical extinction in M 33 is found to be globally low, $A_{V}<0.4 \mathrm{mag}$. Towards HII regions $A_{V} \sim 1$ mag on average even though it can reach values as high as 2 mag towards high surface brightness cores (e.g. Israel \& Kennicutt 1980; Devereux et al. 1997). The moderate values of extinction imply that $\mathrm{H} \alpha$ can be used as an SF tracer globally, but towards individual sources an extinction correction can become necessary.

\section{Large scale structure of stars and dust}

In the present paper, we concentrate on the inner region of M 33 as defined by the Spitzer inner AORs which is outlined by the continuous line in Figs. 1 and 2 for the IRAC and MIPS data, respectively.

In order to help the interpretation of Spitzer data, we first review briefly which are the main emission phenomena that dominate each IRAC and MIPS waveband.

\subsection{Origin of the emission detected by Spitzer}

The stars (mainly Red Giant Branch and/or Asymptotic Giant Branch, according to the age of the population) account for most of the emission in the $3.6 \mu \mathrm{m}$ IRAC band. A mix of stars and very hot dust continuum contribute to the emission in the $4.5 \mu \mathrm{m}$ band. Nevertheless, in discrete sources with negligible continuum (see Sect. 4), the emission seen in both these bands can have a strong component of ionic and molecular lines (e.g., Reach et al. 2006; Williams et al. 2006). In the $5.8 \mu \mathrm{m}$ IRAC band, the stellar contribution decreases and the dust continuum component gets stronger. The longest-wavelength $8.0 \mu \mathrm{m}$ IRAC band still traces hot dust continuum but in most cases, the polycyclic aromatic hydrocarbons (PAH) dominates. If there are no PAHs and low continuum, however, in discrete sources there can be a sizable contribution from two $\mathrm{H}_{2}$ lines and the [Ar III] at $8.9 \mu \mathrm{m}$ (e.g., Hora et al. 2004).

The PAH emission features (mainly concentrated at 3.3, 6.2, $7.7,8.6$, and $11.3 \mu \mathrm{m}$ ) are the optically active vibrational modes of PAH molecules (Leger \& Puget 1984; Allamandola et al. 1989), resulting from internal conversions of energy following absorption of an optical or UV photon. The IRAC bands 3.6, 5.8, and $8.0 \mu \mathrm{m}$ contain PAH emission features at $3.3,6.2$, and $7.7 \&$ $8.6 \mu \mathrm{m}$, respectively (Draine 2003; Churchwell et al. 2004). The $4.5 \mu \mathrm{m}$ channel is the only one with no PAH features, although it contains the bright hydrogen recombination line $\operatorname{Br} \alpha(4.05 \mu \mathrm{m})$.

We investigate the far-infrared (FIR) emission with the MIPS images. The $24 \mu \mathrm{m}$ emission is contributed partly by thermal emission of classic (large) dust grains and partly by very small grains (VSGs). Indeed, VSGs were introduced by Desert et al. (1990) as the carrier of the emission in the $25 \mu \mathrm{m}$ IRAS band. The $70 \mu \mathrm{m}$ emission traces cooler dust, and the $160 \mu \mathrm{m}$ one shows the diffuse cold dust component.

\subsection{Structure of infrared emission in M33}

Figure 1 shows that the stellar component is smoothly distributed everywhere in the disk of M 33. The warm and hot dust traced by the $5.8 \mu \mathrm{m}$ channel smoothly follow the morphological patterns (spiral arms and flocculent structures) of M 33 with a higher concentration in the inner part of the galaxy. Compared with the other components, the $8.0 \mu \mathrm{m}$ contribution shows most clumpy distribution; evidently PAHs are preferentially located along the spiral arms and flocculent structures, rather than being uniformly distributed in the ISM. The very centre of the galaxy gathers all the three contributions: stars, dust and PAHs.

\subsection{The colour images}

To help investigate the large scale structure of M33 as seen by Spitzer we made two sets of colour images: one degraded to the worst resolution of the IRAC channels, and a second set degraded to the resolution of the MIPS $24 \mu \mathrm{m}$ image. We approximated the PRF with a Gaussian and convolved both sets to the resolution of the worst image in the set $(8 \mu \mathrm{m}$ and $24 \mu \mathrm{m}$, respectively).

Figure 3 shows the 3.6/4.5 ratio (left panel) and 4.5/8.0 ratio (right) of M 33. The 3.6/4.5 shows a remarkably constant ratio $(\sim 1.7)$ over almost the entire galaxy, confirming the statement by Pahre et al. (2004), who find that for each galaxy, the [3.6]-[4.5] colour is nearly constant with radius and consistent with stellar photospheric emission. The 5.8/8.0 ratio (not shown in Fig. 3) is also approximately constant $(\sim 0.5)$, similarly to other galaxies such as NGC 300 by Helou et al. (2004), even though it might not be a general feature. In both the 5.8 and $8.0 \mu \mathrm{m}$ bands there is an increase of the diffuse emission relative to the 3.6 and $4.5 \mu \mathrm{m}$ bands, as in our Galactic plane. The $8.0 / 24$ colour (not shown) also does not show any evident radial gradient or prominent structure and is practically constant across the disk. Similarly to the 5.8/8.0 ratio, this result points out the overall constancy in the ratio of PAHs and warm dust emission in this galaxy.

On the other hand, the 4.5/8.0 images (right panel of Fig. 3) and the 3.6/5.8 ratio (not shown) both retain the structural pattern of M 33, tracing the inner features, the two main spiral arms and the filamentary structure of the disk. In the first case, this is probably due to the predominant PAH features in the spiral arms and filaments; in the second, the ISM as reflected in the $5.8 \mu \mathrm{m}$ band is emerging relative to the stars at $3.6 \mu \mathrm{m}$. The $8.0 \mu \mathrm{m}$ emission along the spiral arms and along filaments surrounding the dimmer star forming regions at large galactocentric distances become prominent.

We also constructed an IRAC three colour image of M 33 by subtracting the stellar component in the shorter-wavelength images from the 5.8 and $8.0 \mu \mathrm{m}$ ones. To remove the stellar contribution from the 5.8 and $8.0 \mu \mathrm{m}$ images, we used the recipe by Helou et al. (2004); Pahre et al. (2004); Calzetti et al. (2005); Perez-Gonzalez et al. (2006). The 3.6 and $4.5 \mu$ m images were combined assuming colours appropriate for an M0 III star ([3.6]-[4.5] $=-0.15$ in Vega mag). The final combination is shown in Fig. 4 and displays the stars in blue, the star-subtracted 

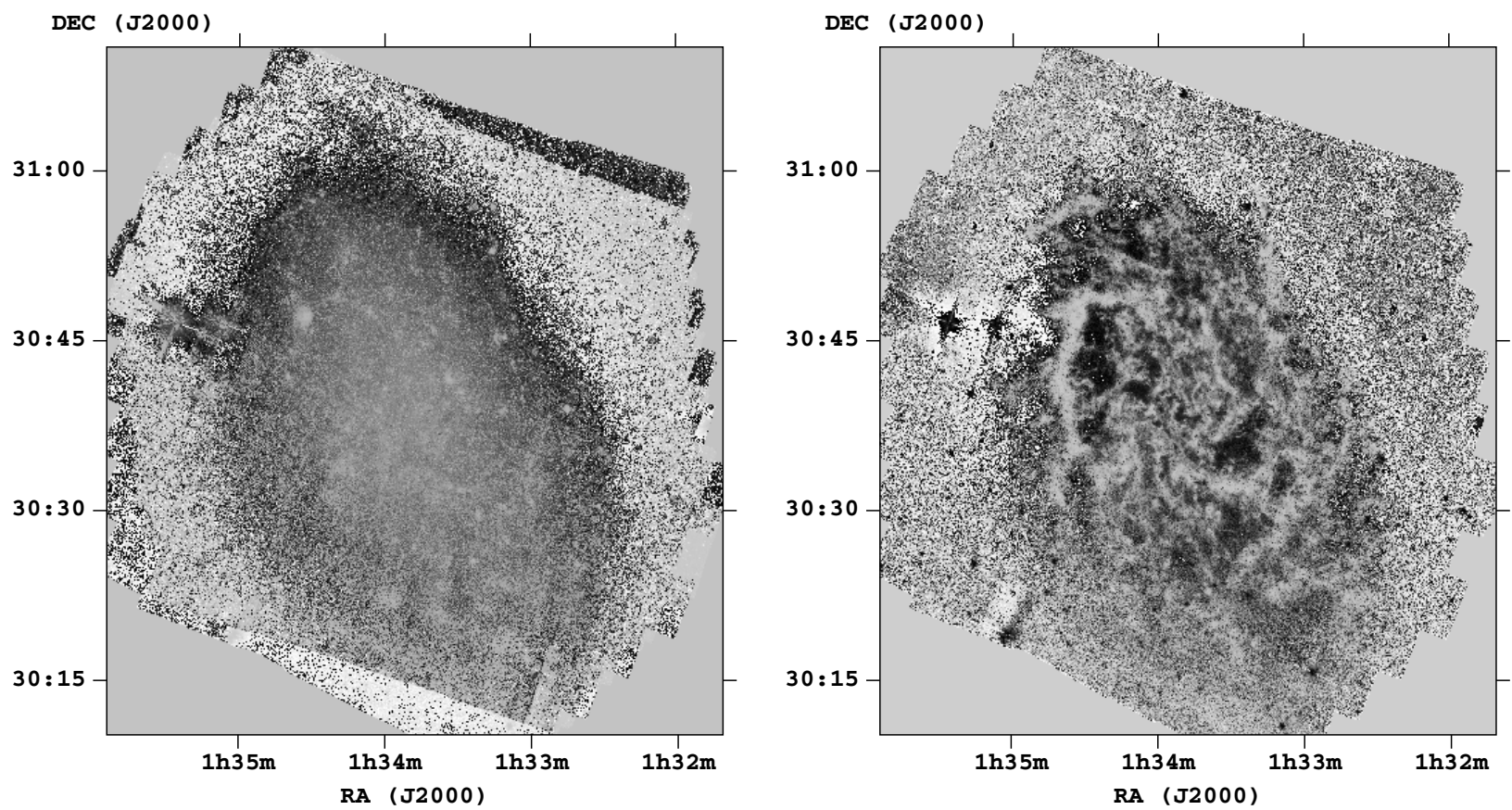

Fig. 3. Colour images representing the ratio of the 3.6 over $4.5 \mu \mathrm{m}$ images (left) and 4.5 over $8.0 \mu \mathrm{m}$ (right). These images have a common resolution roughly equivalent to that in the $8.0 \mu \mathrm{m}$ image. White corresponds to low colour ratios, and black to high ones.

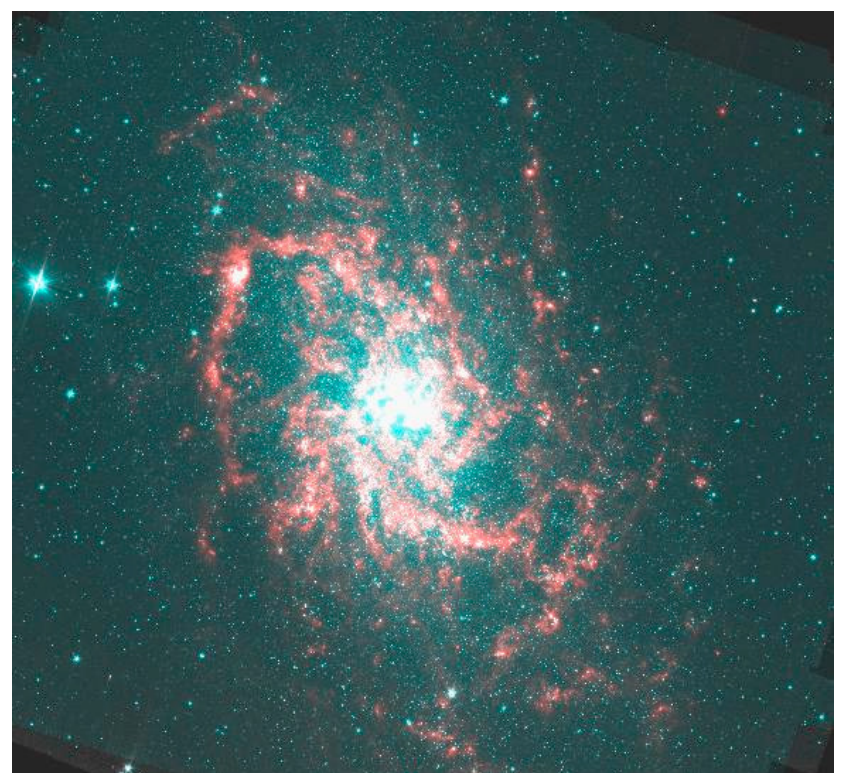

Fig. 4. M33 three colour image, composite of the central region of the complete IRAC data. Blue is a weighted mean of 3.6 and $4.5 \mu \mathrm{m}$, representing the evolved stellar population. Green depicts the $5.8 \mu \mathrm{m}$ light after removal of stellar emission, and red traces the $8.0 \mu \mathrm{m}$ channel also corrected for stellar emission. (See the electronic edition of the Journal for a colour version of this figure.)

$5.8 \mu \mathrm{m}$ in green and the star-subtracted $8.0 \mu \mathrm{m}$ in red. The dominance of the $8 \mu \mathrm{m}$ emission in the spiral arms is clearly revealed by Fig. 4. The underlying stellar disk is also evident; the excess of stellar light in the centre has been pointed out by previous near-infrared ground observations (Regan \& Vogel 1994), and is related to the possible presence of a bulge or bar component.

\subsection{Distribution of cool dust}

Over the last fifteen years, many studies have been devoted to the FIR emission of M 33 using IRAS and ISO. Rice et al. (1990) observed M 33 with IRAS at $12,25,60,100 \mu \mathrm{m}$ and found a striking correlation between the spatial IR structure and the HII regions. They estimated that $75 \%$ of the integrated IR emission arises from stars younger than a few times $10^{8} \mathrm{yr}$. The close correspondence between the $\mathrm{H} \alpha$ image and the FIR $(60$ and $100 \mu \mathrm{m})$ morphology was also noted by Devereux et al. (1997). Hippelein et al. (2003) studied M 33 with ISOPHOT at 60, 100 and $170 \mu \mathrm{m}$, and concluded that the FIR emission is composed of a superposition of two components: i) a warm one, found in spiral arms and SF regions, heated by UV radiation from OB stars, contributed by dust at about $45 \mathrm{~K}$; and ii) a cold one around $16 \mathrm{~K}$ which is smoothly distributed over the disk and heated by diffuse interstellar radiation.

More recently, Hinz et al. (2004) used MIPS observations of M 33 to investigate the origin of the FIR emission, and found that the emission in the 24 and $70 \mu \mathrm{m}$ MIPS images follows closely the structure of the ionised gas, suggesting heating by hot, ionising stars. The overlays of Fig. 6 (see Sect. 3.5) show the link between 24 and $\mathrm{H} \alpha$, i.e. between the hot dust emission and the formation sites of massive stars (see also Tabatabaei et al. 2007a). This link is also evident by comparing the $\mathrm{H} \alpha$ and $70 \mu \mathrm{m}$ emission maps. Moreover, comparing thermal and nonthermal radio images of M 33 with MIPS suggests that the 24 and $70 \mu \mathrm{m}$ emission is more closely associated with the thermal radio component than with the non-thermal one (Tabatabaei et al. 2007a). Both the 24 and the $70 \mu \mathrm{m}$ emission originates in fact from warm dust associated with massive star formation. We discuss in more detail the connection between $24 \mu \mathrm{m}$ discrete sources and $\mathrm{H} \alpha$ knots below.

A more diffuse component emission at $160 \mu \mathrm{m}$ is evident when comparing it with the $24 \mu \mathrm{m}$ map. Cold dust heated by a diffuse interstellar radiation field clearly contributes to the longer wavelength MIPS band. 


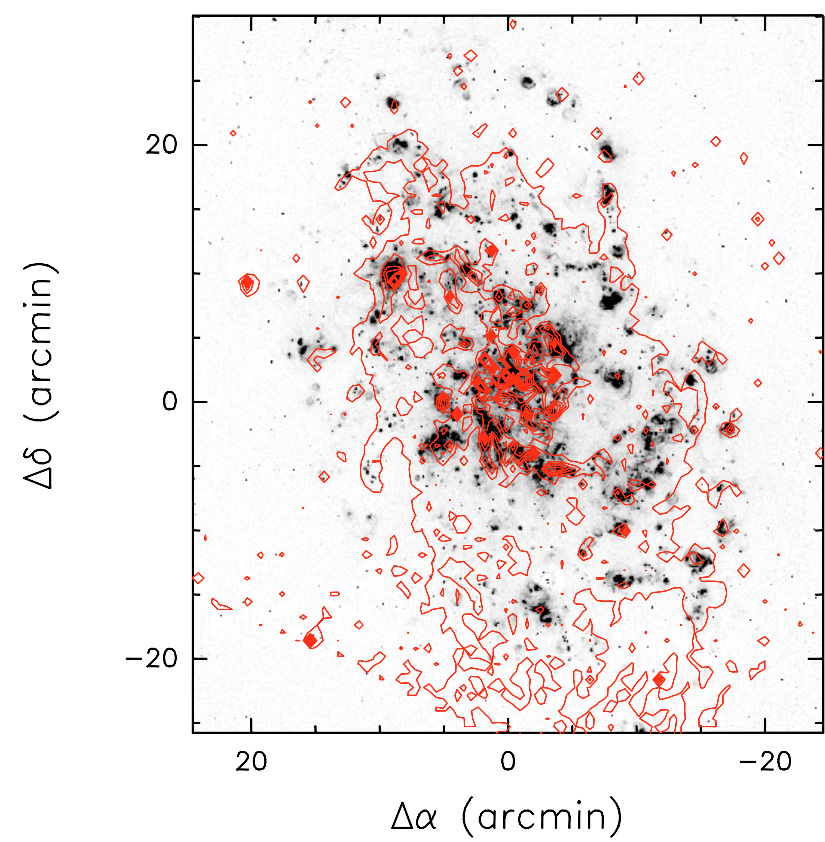

Fig. 5. H $\alpha$ image of M 33 with the over-plot of $8.0 \mu \mathrm{m}$ contours.

\subsection{Comparison with $\mathrm{H} \alpha$}

In Figs. 5 and 6 , we show the 8.0 and $24 \mu \mathrm{m}$ contours on the $\mathrm{H} \alpha$ image. Other than the two brightest HII regions (NGC 604 and IC 133), the highest levels of $24 \mu \mathrm{m}$ emission are concentrated in the inner part of the galaxy, and the $24 \mu \mathrm{m}$ emission mainly follows the spiral arms. Notably, not all the $\mathrm{H} \alpha$ bright spots have bright $24 \mu \mathrm{m}$ counterparts. Moreover, the centre of the $24 \mu \mathrm{m}$ emitting sites can be shifted with respect to the centre of the $\mathrm{H} \alpha$ emission sites. This might indicate that some of the optical emission from the HII regions is obscured by dust or may be due to different intrinsic structures related to the evolution of HII regions. Of particular interest are the $24 \mu \mathrm{m}$ emission spots not associated with $\mathrm{H} \alpha$ emission, which could trace deeply embedded HII regions, or a different population of objects. In Fig. 5, we can see the same general trends with a concentration of the $8 \mu \mathrm{m}$ emission in the centre and along the spiral arms of the galaxy. At larger radii, the $8 \mu \mathrm{m}$ emission becomes more diffuse respect to the $24 \mu \mathrm{m}$ component which matches better the bright $\mathrm{H} \alpha$ knots. At $8 \mu \mathrm{m}$, the southern arm shows a much more concentrated emission than the northern arm. A similar trend has also been found for the molecular gas: giant molecular clouds and diffuse molecular gas emission follow the pattern of the arm in the south as traced by $\mathrm{H} \alpha$ much more closely than in the north (Engargiola et al. 2003; Heyer et al. 2004).

\section{The IR emission of nebulae in M 33}

We used six catalogues of three classes of known-type sources in M33: three catalogues of HII regions selected at different wavelengths, two catalogues of SNRs, and one catalogue of PNe:

1. IR HII regions: 28 sources, Giveon et al. (2002), Willner \& Nelson-Patel (2002);

2. Radio HII regions: 40 sources, Gordon et al. (1999);

3. Optical HII regions: 78 sources, Magrini et al. (2007b);

4. Optically selected SNRs: 98 sources, Gordon et al. (1998);

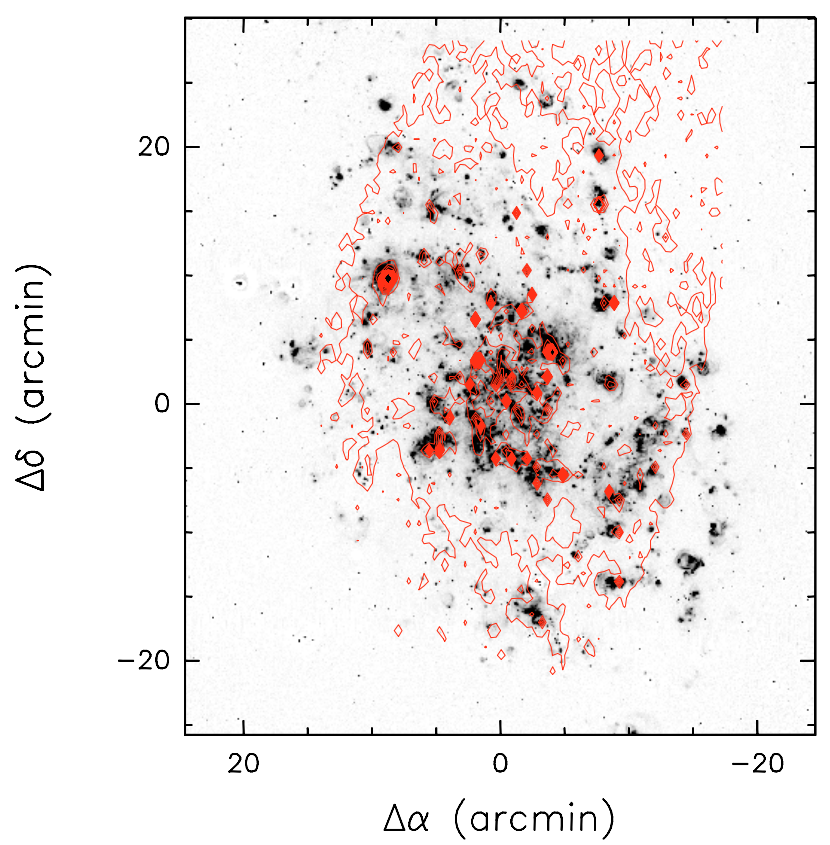

Fig. 6. $\mathrm{H} \alpha$ image of M 33 with the over-plot of $24 \mu \mathrm{m}$ contours.

5. Radio selected SNRs: 30 sources, Duric et al. (1995), Gordon et al. (1999);

6. PNe: 152 sources Ciardullo et al. (2004).

The properties of these will be compared to our catalogue of $24 \mu \mathrm{m}$-selected sources in Sect. 6.

We have selected our sample of HII regions from three different catalogues, which include HII regions of different morphologies, locations within the galaxy, and brightness. Our IR sample was obtained by merging the sources observed by Willner \& Nelson-Patel (2002) and Giveon et al. (2002). It includes in total 28 sources located in three chains, to the east, west, and south of the galaxy nucleus. Our radio sample comprises $40 \mathrm{HII}$ regions with 20-6 cm spectral index $\left(v^{\alpha}\right) \alpha>-0.2$ as catalogued by Gordon et al. (1999), and extends out to $20^{\prime}$ galactocentric radii. A list of 78 optically selected HII regions, from narrow band images and with known optical spectra, was kindly provided to us by L. Magrini (Magrini et al. 2007b). Such regions are in general much fainter than the IR or radio selected regions and are located preferentially at large galactocentric radii.

To probe dust emission during the final stages of stellar evolution, we selected two samples of SNRs, one defined optically and one defined in the radio. The optical sample (Gordon et al. 1998) contains 98 SNR candidates selected primarily on the basis of optical emission-line ratios. The radio sample of SNRs contains 30 (radio-bright) sources appearing both in Duric et al. (1995) and Gordon et al. (1999). Their selection criteria are effective at finding middle-aged remnants. In fact, since their SNRs have diameters in the range 20-48 pc, most of the SNRs should be in the Sedov-Taylor expansion stage. The contamination in their sample is estimated to be less than 1 , but there are 20 SNRs embedded within HII regions. Still, several remnants are associated with nearby HII regions and some are embedded in them.

Finally, we adopt the catalogued PNe of Ciardullo et al. (2004). As a result of a photometric and spectroscopic survey, they identified $152 \mathrm{PNe}$ candidates in M 33. By comparison with Magrini et al. (2001), the contamination by HII regions or SNRs is estimated at $\sim 22 \%$. 


\subsection{Photometric methodology}

We have six source lists of known type, for a total of more than 400 objects distributed over the entire disk of M 33. Our aim is to use the IRAC/MIPS-24 colours of these as empirical diagnostics to infer the dust properties in various kinds of nebulae. This is done by comparing their IR colours with the theoretical colour diagrams (see Sect. 5).

To these source lists we then add the $24-\mu \mathrm{m}$ selected sample and we shall infer the nature of these sources by comparing their IR colours with those of known-type of sources (see Sect. 6). We are also interested in the $\mathrm{H} \alpha$ emission of our objects, in particular for the $24-\mu \mathrm{m}$ selected sample and therefore we need to perform photometry on the $\mathrm{H} \alpha$ image. Therefore, we must perform photometry in a total of six bands (4 IRAC, MIPS-24, and $\mathrm{H} \alpha$ ). We merge the IR photometry, and consider only those sources which have measurable emission in all the IR bands examined, in order to place them on our diagnostic colour diagrams.

The proximity of M33 combined with the superb resolution of Spitzer makes photometry of individual sources in the galaxy challenging. Isolating sources is difficult, because of the extremely crowded fields, especially the inner disk and circumnuclear regions. Moreover, we expect that many of the objects (e.g., HII regions and SNRs) will be extended, so that we cannot accurately perform point-source fitting and subtraction with point-response functions. In addition, many classes of sources are not prodigious emitters in the mid-infrared (MIR), which further complicates the automatization of the photometric procedures. Hence, we investigated many photometric schemes before finally arriving at a reliable solution.

Following Calzetti et al. (2005), we first attempted aperture photometry of the sources in our lists. The initial centres were taken from the source lists described in Sect. 4, and the centre of the virtual aperture was then allowed to vary up to a certain distance from the initial position. While this is a viable solution in other situations, it turned out not to be feasible in M33. First, the definition of the fiducial aperture should depend on source extent, but this differs among the source classes, so it was not clear how to define the limiting aperture. This was an especially vexing problem for the wavelengths in which a given source does not have measurable emission, since the automatic centring routines did not converge. Second, the determination of the background was extremely problematic because of the variable degree of crowding and the variable degree of diffuse emission. It was also unclear how to define either "regional" or "global" backgrounds in the case of M33. Thus, we were forced to find another solution.

We then attempted elliptical cross-section Gaussian fitting of sources (e.g., Calzetti et al. 1995). The fitting box was fixed to a series of dimensions (from 25 to 10 pixels), and the centre, the orientation, the width, and a constant background level were left free to vary. As before, the initial centres were taken from the source lists. The larger fitting boxes allowed the centre to wander too much from the initial position. Hence, the source identified by the fitting routine frequently did not correspond to the source in the list, although reasonably accurate backgrounds were obtained when checked against the source surroundings. The smaller fitting boxes better constrained the centre, but in crowded fields gave a background which was much too high. This resulted in gross underestimations of the total flux, especially when the source was rather faint and comparable to the estimated background. An additional problem encountered with Gaussian fitting was its unsuitability for multiple sources. Many of the sources in our lists are in truth "clusters" of smaller sources when examined with the Spitzer spatial resolution and at wavelengths different from those in which the sources were defined. These problems taken together led us to distrust this method for a large fraction of sources in our lists.

To overcome these shortcomings, we finally devised a "hybrid" solution, which optimally combines the photometry from Gaussian fitting and that from isophotal photometry. This last was accomplished with SExtractor (Bertin \& Arnouts 1996) which is able to deal with very large images automatically, and handles reasonably accurately a wide variety of object shapes and sizes. The idea was to calculate the total flux integrated over an irregularly shaped aperture containing flux levels above some isophotal limit. The hybrid method consists of three steps: (1) perform Gaussian fitting of all sources as described in the previous paragraph; (2) perform SExtractor photometry as described below; (3) merge the two sets of photometry according to an algorithm which decides which photometric measurement to prefer, in order to have one measurement per source.

For (1), we adopted a fitting box of 10 pixels; this was the best compromise between centre wandering and background level, as the photometry was typically centred on a position relatively close to the initial one, although with a potentially spuriously low flux because of the background determination.

In practice, for (2), we used the ASSOCIATIVE feature of SExtractor, and input our source lists with initial positions. The initial SExtractor photometry consisted in those sources extracted at a $10 \sigma$ level above the background, at a distance within 15 pixels $(\equiv \Delta r)$ from the initial position. These two values resulted to be the best compromise between source association and source identification. Lower values of signal-to-noise ratio $(<10 \sigma)$ resulted in more sources being identified, but were in the mean farther away. The background for the SExtractor photometry was defined to be the local median of the image in a 64-pixel box centred on the source.

Step (3), the merging of the Gaussian fitting and SExtractor photometry, was accomplished by examining the radial distance of each measurement from the initial position. When the SExtractor centre was within $\Delta r$ of 5 pixels (in radius), and as long as it was within a factor of two of the Gaussian fitting centre, it was preferred over the Gaussian method. We experimented with a few values of $\Delta r$ and a few values of the relative ratio of the radial distances, and concluded that this gave the best results overall. Indeed, it appears that this hybrid approach overcomes the problems of underestimating source fluxes because of background estimates, and successfully calculates the total flux even when sources are in fact multiple. Indeed, the SExtractor photometry and the Gaussian fitting results are fairly well correlated except for low fluxes with high background where Gaussian fitting tends to underestimate the true flux, and high fluxes for extended or clustered sources.

\subsection{Detection rates}

The detection statistics are illustrated in Fig. 7 and Table 1. The matching radius $\Delta r_{\max }$ for the coincidence of sources, was determined by examining the scatter on the diagnostic diagrams, see Sect. 5 . In the end we adopted a value $\Delta r_{\max }=3$ pixels (about $14 \mathrm{pc}$ ), which is sufficiently large to accommodate possible astrometric uncertainties.

The positions of catalogued sources with photometry in all four IRAC bands are shown in Fig. 8, on the IRAC $4.5 \mu \mathrm{m}$ image of M33. The upper panel refers to HII regions and we note that the optically selected sample is mainly in the outer regions of the galaxy, while the IR and radio selected HII regions are in 


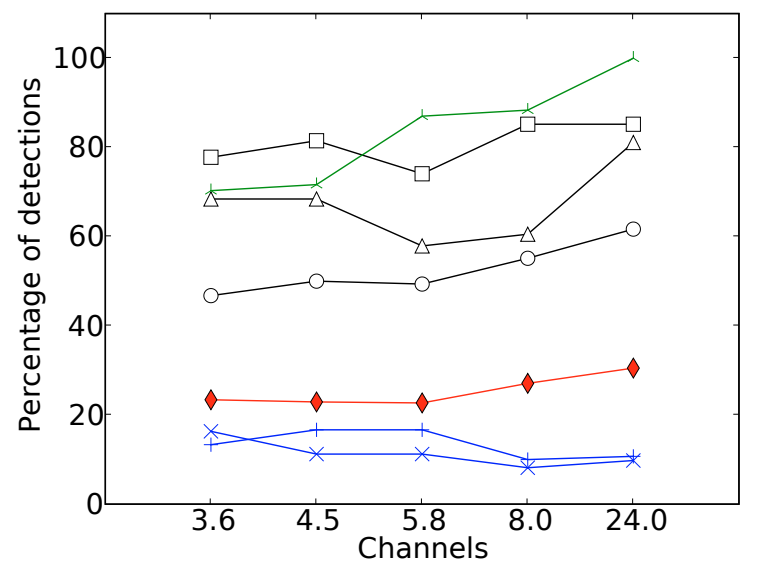

Fig. 7. Detection rate in the different wavebands. Open symbols represent the HII regions selected in IR (squares), radio (triangles) and optical (circles). The (blue) crosses represent the optically selected SNRs and the (blue) plus signs the radio selected SNRs. The filled (red) diamonds represent the PNe. The (green) tripods represent our $24 \mu \mathrm{m}$ sources.

Table 1. Detection rate in all four IRAC and IRAC+MIPS-24 bands.

\begin{tabular}{cccc|ccc}
\hline \hline & IRAC & Total & $\%$ & IRAC+MIPS-24 & Total & $\%$ \\
\hline ISO & 17 & 27 & $61 \%$ & 15 & 27 & $54 \%$ \\
Hra & 18 & 38 & $45 \%$ & 18 & 37 & $45 \%$ \\
HII & 27 & 77 & $35 \%$ & 19 & 60 & $32 \%$ \\
SNo & 3 & 98 & $3 \%$ & 2 & 92 & $2 \%$ \\
SNR & 1 & 30 & $3 \%$ & 1 & 28 & $3 \%$ \\
PNe & 9 & 141 & $6 \%$ & 4 & 118 & $3 \%$ \\
$24 S s$ & 306 & 515 & $59 \%$ & 306 & 515 & $59 \%$ \\
\hline
\end{tabular}

the inner parts of M33. The lower panel shows the locations of PNe and SNRs. The single detection of the radio-selected SNR coincides with an optically-selected one.

\subsubsection{HII regions}

The detection rate of HII regions depends on the waveband and on the catalogue. As expected, the brighter HII regions (radio and IR sample) are detected at a higher rate than the faint ones (optically selected catalogue). The detection rate for bright regions increases even more if we loosen the strict requirement about the centre displacement. For large HII regions in fact the centre of the IR emission can be displaced by more than 3 pixels from the nominal position, and hence not accepted as coincident. The lower resolution in the $24 \mu \mathrm{m}$ band makes objects rounder, and the Gaussian fitting is often more accurate than in the 5.8 and $8.0 \mu \mathrm{m}$ band. This partly accounts for the higher detection rate in the $24 \mu \mathrm{m}$ MIPS wavelength. But, there is a general tendency for the HII regions to be less visible in the shortest wavelengths (3.6 and $4.5 \mu \mathrm{m}$ ) than in the 8.0 and $24.0 \mu \mathrm{m}$ channels. The detection rate at $5.8 \mu \mathrm{m}$ always shows a drop, with respect to the adjacent channels (see Fig. 7).

\subsubsection{Supernovae remnants}

We found a low rate of IR counterparts for the SNRs identified in optical and in radio. The percentage of detections is about $15 \%$ for both samples. Four SNRs were detected in at least one band: two were detected at all wavelengths, and other two only at $4.5 \mu \mathrm{m}$. The four detected SNRs have radii of $\sim 8-16 \mathrm{pc}$, while the two undetected ones have radii greater than $20 \mathrm{pc}$

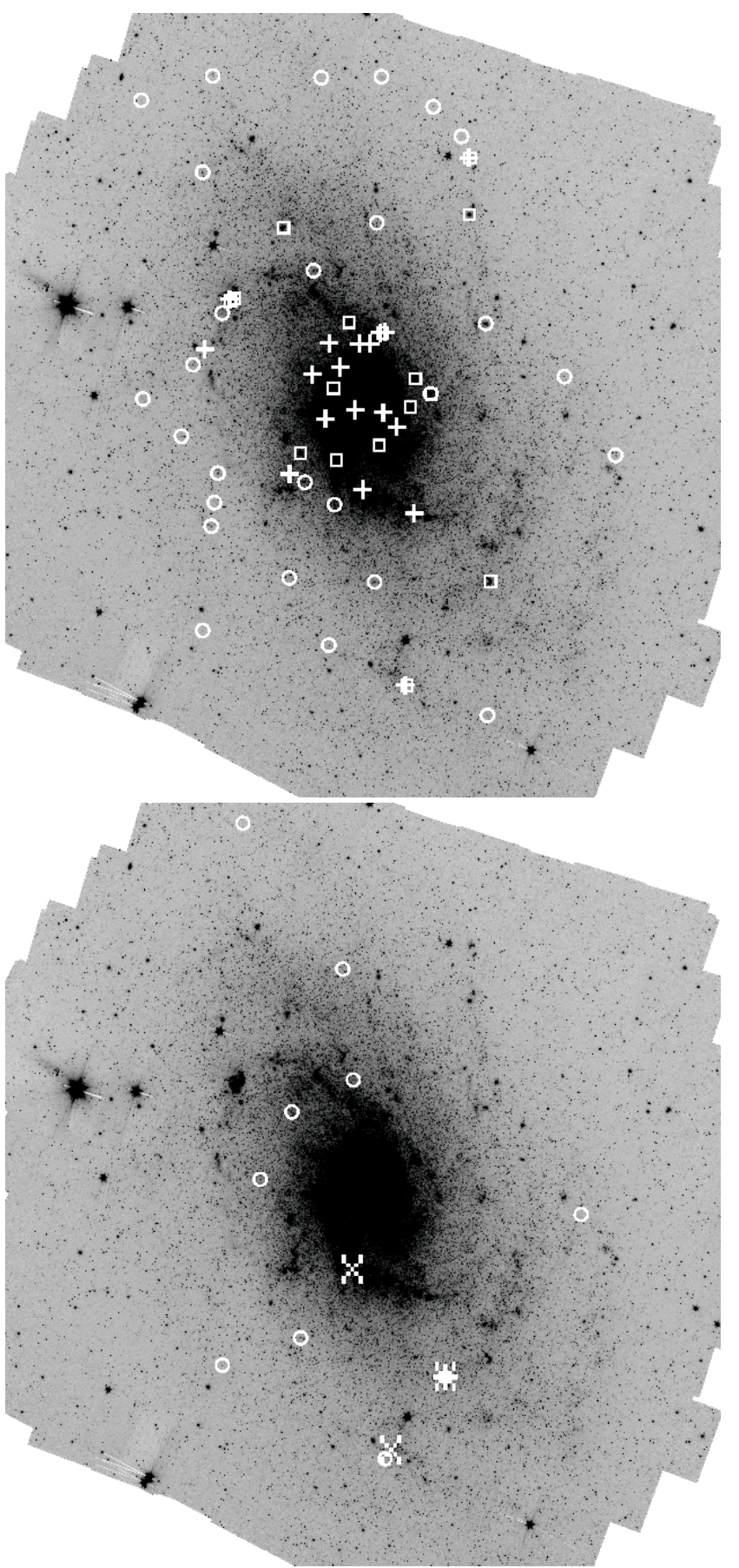

Fig. 8. The position of catalogued sources with photometry in all four IRAC bands superimposed on the $4.5 \mu \mathrm{m}$ IRAC image. The upper panel shows the IR selected (open squares), radio selected (pluses) and optically selected (open circles) HII regions. The lower panel shows the locations of the PNe (open circles), optically selected (crosses) and radio selected (pluses) SNRs. The images are about $1^{\circ}$ on a side, north is up and east to the left.

and may be of a lower surface brightness. The low number of SNR detections is consistent with previous IRAS studies trying to find Galactic SNR counterparts in the IR: 17\% (Arendt 1989) and $18 \%$ (Saken et al. 1992). Also, using IRAC, Reach et al. (2006) found only 18 IR counterparts out of 95 SNRs in the inner Galaxy. Very few Galactic SNRs were detected by MSX, the Midcourse Space Experiment (Cohen et al. 2006). In the Large Magellanic Cloud, Borkowski et al. (2006) found no emission 
from type Ia SNRs and Williams et al. (2006) did not detect the two largest SNRs of their sample in any of the IRAC or MIPS wavebands.

In our samples, the detection rate is also about $30 \%$ less in the 8.0 and $24 \mu \mathrm{m}$ bands with respect to the shorter IRAC wavelengths. This might be due to the destructive effect of the blast wave on the smaller grains; PAHs can already be razed in slow shocks and they are never detected in dense, shocked clumps (Reach et al. 2002). The emission seen at 3.6 and $4.5 \mu \mathrm{m}$ is likely due to ionic and molecular lines, such as $\operatorname{Br} \alpha 4.1 \mu \mathrm{m}$ (Reach et al. 2006; Williams et al. 2006). The environment of the SNRs should have a strong influence on their IR detection rate: remnants interacting with a denser external medium should be more easily detectable. If PAHs in the surrounding ISM are destroyed by nearby supernova events (Gorjian et al. 2004), they will not emit at $8.0 \mu \mathrm{m}$ (nor contribute to 3.6 and $5.8 \mu \mathrm{m}$ ), which could explain the quite low rate of detection of a remnant in all 4 bands.

\subsubsection{Planetary nebulae}

The detection rate of $\mathrm{PNe}$ is about $25 \%$ for the three shorter IRAC wavelengths and then rises steeply to reach more than $50 \%$ in the $24 \mu \mathrm{m}$ band. This is consistent with the result by Cohen \& Barlow (2005) who detect PAH emission in less than half of a sample of 43 Galactic PNe. In addition, Hora et al. (2004) claim that the IRAC colours of PNe are red; despite typically negligible continua, they are bright in the $8.0 \mu \mathrm{m}$ band probably because of a sizable contribution from the two $\mathrm{H}_{2}$ lines and an [Ar III] line at $8.99 \mu \mathrm{m}$.

\subsection{Spectral energy distributions}

The average IR Spectral Energy Distribution (SED) for our different lists of objects is shown in Fig. 9. HII regions, either IR or radio selected, display the highest fluxes, about two order of magnitudes above the faintest in the list, that is the PNe. The HII optical sample instead, largely consisting of quite faint regions, sits at lower values, in between the two samples of SNRs. The steep rise between 8.0 and $24 \mu \mathrm{m}$ of all the HII regions samples, implies that HII regions are invariably associated with warm dust. SNRs and PNe show milder slopes, their SEDs being almost flat, or even declining as for optically selected SNRs. This last feature reveals changes of the ISM characteristics related to SN events.

In HII regions, the drop between 3.6 and $4.5 \mu \mathrm{m}$ can be accounted for by the photospheric contribution of young stars in the two shortest wavelength IRAC channels or perhaps line emission. Candidate lines in the $3.6 \mu \mathrm{m}$ band include [Fe III] $3.229 \mu \mathrm{m}$, and [Co III] $3.492 \mu \mathrm{m}$ (Gerardy et al. 2007), but line emission can be important in other bands as well as long as there is little continuum emission. This drop has also been detected by Mercer et al. (2004); Gorjian et al. (2004); Jones et al. (2005); Williams et al. (2006); Gerardy et al. (2007). The absence of the $4.5 \mu \mathrm{m}$ drop in the means of radio selected HII's is due primarily to a single object with a high $4.5 \mu \mathrm{m}$ flux.

\section{Diagnostic diagrams}

\subsection{Theoretical diagnostic diagrams for IRAC and IRAC/MIPS flux ratios}

To better understand the IRAC/MIPS colour diagrams of discrete sources, we assembled a series of models with different physical parameters, as described more in detail below. The IR colours

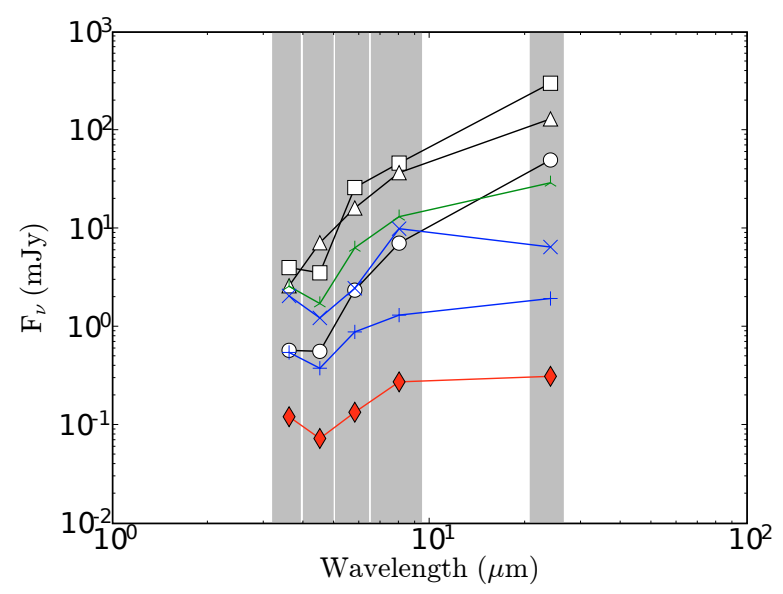

Fig. 9. Mean IR SEDs of the different kind of sources. Open symbols represent the HII regions selected in IR (squares), radio (triangles) and optical (circles). The (blue) crosses represent the SNRs optically selected and the (blue) pluses the SNRs radio selected. The filled (red) diamonds represent the PNe. The (green) tripods represent the $24 \mu \mathrm{m}$ unknown sources. The grey vertical stripes depict the widths of the IRAC and MIPS-24 filters.

are derived by convolving the model spectra with the IRAC and MIPS-24 filter response curves, as provided by the Spitzer Science Center web page. The colours are here computed as ratio of flux densities (in Jy, i.e. per unit frequency).

\subsubsection{HII regions with Cloudy}

HII regions are possibly the most prominent class of sources in our images. We modelled them with version 05.07 of Cloudy, last described by Ferland et al. (1998) (see also Ferland 2003). The central star was taken to be a $\log g=4$ Kurucz model (Kurucz 1979) at $T_{\text {eff }}=37000 \mathrm{~K}$, as modified by Rubin to better account for the stellar flux in the model atmospheres between $41 \mathrm{eV}$ and $54 \mathrm{eV}$. In all cases, we assumed a stellar ionising luminosity of $10^{49} \mathrm{~s}^{-1}$, spherical geometry, constant gas density, a covering factor of 0.005 , and a metal abundance of $50 \%$ solar.

Grains were "turned on" in the Cloudy models so that heating and cooling of the grains and the gas are calculated in a self-consistent way. A standard PAH distribution is included in the grain population, with treatment of photoelectric heating and collisional processes (see Ferland et al. 1998). We did not explicitly introduce a photo-dissociation region (PDR) in the calculations.

We accounted for the range in typical HII region parameters by varying the outer radius and the total hydrogen density so as to roughly follow the well-known size-density $n_{e} \sim D^{-1}$ relation for HII regions (Garay \& Lizano 1999; Kim \& Koo 2001). Four models were calculated with the following pairs in size-density space with density given first in $\mathrm{cm}^{-3}$ followed by outer radius in pc: $(1000,2) ;(100,10) ;(20,30) ;(10,60)$.

The resulting spectra are shown in Fig. 10, where the (blue) short-dashed line shows $(100,10)$; the (cyan) dotted line $(20,30)$; the (green) long-dashed line $(10,60)$; and the (red) solid line $(1000,2)$. It can be seen from the figure that varying the density by a factor of 100 drastically changes the PAH emission and its dominance relative to the continuum. Since the PAHs in most HII regions originate in the surrounding PDR rather than in the ionised region itself (e.g Whitney et al. 2004; Churchwell et al. 2004; Dopita et al. 2006), the simulations may not be truly representative of HII regions unless a PDR is absent. However, 


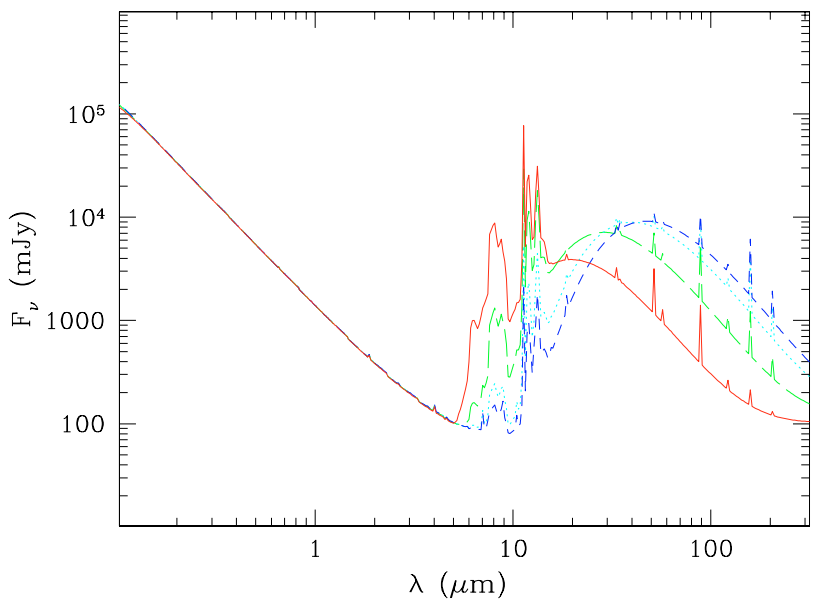

Fig. 10. Spectra obtained with Cloudy code, for the four models. The parameter pairs in size-density space with density given first in $\mathrm{cm}^{-3}$ followed by outer radius in pc are represented by the (blue) short-dashed line for $(100,10)$; the (cyan) dotted line $(20,30)$; the (green) long-dashed line $(10,60)$; and the (red) solid line $(1000,2)$.

they clearly span a wide range in IR peak wavelength and $\mathrm{PAH}$ strength, so should be adequate for helping to interpret the IRAC/MIPS24 diagnostic diagrams.

\subsubsection{Embedded HII regions with DUSTY}

The HII regions we have modelled with Cloudy are basically optically thin, because we have not performed multiple iterations on the solution. Moreover, even with iteration, optical depth effects in the dust itself are not taken into account in the Cloudy formalism. Hence, to better model compact and ultra-compact HII regions which are expected to be embedded in an opaque dust cocoon, we have run a series of models with DUSTY (Ivezic \& Elitzur 1997). DUSTY solves semi-analytically the radiative transfer physics in a dust envelope by exploiting the self-similar nature of the problem. The dust is located in a shell external to the HII region itself. The program allows for a variety of spherical shell configurations, and provides a realistic spread of dust temperatures as the radiation field changes with distance from the source. At the same time, this code suffers from some limiting assumptions, such as spherical symmetry, no treatment of stochastic processes for PAH emission, and the decoupling of the gas from dust. Nevertheless, for our purposes, it is a powerful tool with which to investigate the dust continuum emission from young dust-enshrouded stars and star clusters.

With DUSTY, it is necessary to specify the temperature at the inner radius of the dust shell, $T_{\text {in }}$ which, for a given incident radiation field, determines the inner radius of the region, $R_{\mathrm{in}}$. The radial distribution of the dust is described through the ratio of the outer radius to the inner one, $Y_{\text {out }}\left(\equiv R_{\text {out }} / R_{\text {in }}\right)$, and the powerlaw exponent $p$ describing the radial fall-off $R^{p}$. In all cases, we adopted a standard "MRN" size distribution (Mathis et al. 1977), and a standard dust composition (Draine \& Lee 1984). Finally, we must specify the radial optical depth $A_{V}$ which, for a given geometry, fixes the dust mass. The DUSTY models were calculated for 10 values of $A_{V}$, ranging logarithmically from 0.1 to 100 (specifically $0.1,0.215,0.464$ for each decade in $A_{V}$ ). We adopted single-zone models, with only one stratum in the shell.

Two temperatures were input for $T_{\mathrm{in}}, 300 \mathrm{~K}$ and $600 \mathrm{~K}$, which correspond roughly to the range observed in extragalactic HII regions (Hunt et al. 2005). Because of the non-negligible
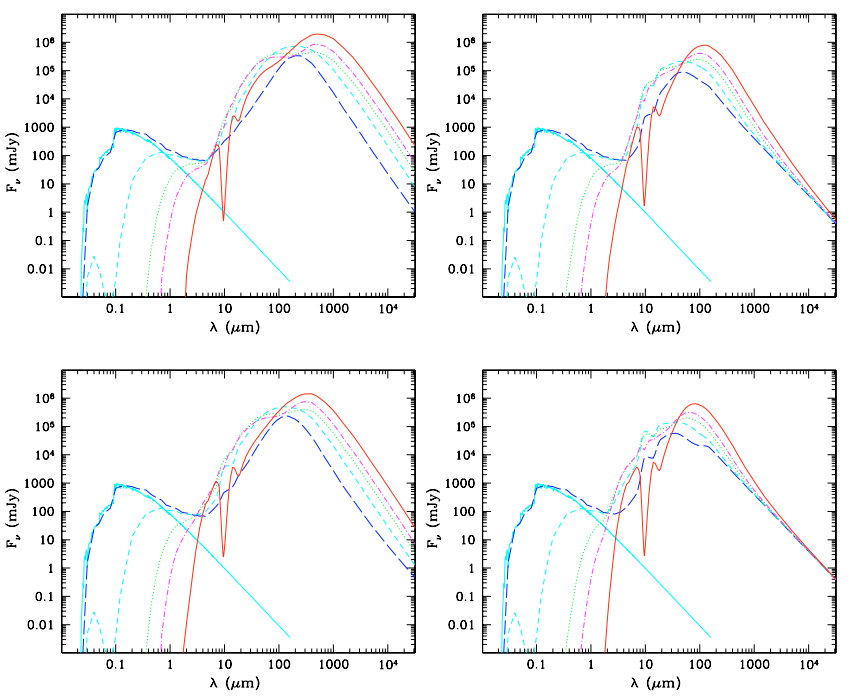

Fig. 11. The resulting DUSTY spectra. We plot only 5 values of $A_{V}$, namely $0.1,2.15,10.0,21.5$, and 100 (shown as long-dashed, shortdashed, dotted, dot-dashed, and solid lines, respectively). Also shown (as a heavy solid line which ends at $\lambda \sim 100 \mu \mathrm{m}$ ) is the input stellar spectrum of a $3 \mathrm{Myr}$ starburst from SB99. The upper panels show $T_{\text {in }}=$ $300 \mathrm{~K}$, and the lower ones $T_{\mathrm{in}}=600 \mathrm{~K}$. The left panels show thick shells $\left(R_{\text {out }}=10000\right)$, and in the right panels thin ones $\left(R_{\text {out }}=100\right)$.

thickness of the dust shell, such relatively high temperatures at its inner rim are also required to explain the average temperatures observed in Galactic HII regions (Conti \& Crowther 2004; Giveon et al. 2007). We fixed the power-law exponent $p$ to -0.2 as a compromise between uniform regions and those with a steeper fall-off. This exponent strongly affects the amount of cool dust in the shell, but does not significantly influence the MIR portion of the spectrum that we are examining with the IRAC/MIPS-24 diagnostic diagrams. The thickness ratio $Y_{\text {out }}$ varied from 10 to $10000 ; Y_{\text {out }}$ also controls the amount of cool dust in the shell as thicker shells have proportionately more cool dust.

To model the exciting star cluster input to DUSTY, we incorporated the Starburst 99 simulations (SB99, Leitherer et al. 1999). Because we are interested in young, dusty star clusters, a single age of 3 Myr was used. SB99 takes into account the nebular continuum emission which can dominate the near-infrared ( $2 \mu \mathrm{m}-4 \mu \mathrm{m}, \mathrm{NIR})$ spectral range, and thus could in principle significantly influence our IRAC colour diagrams (see below). In practice, the slope of the input spectrum of a 3 Myr starburst is quite similar to the star used for the Cloudy simulations, at least down to $0.1 \mu \mathrm{m}$, so we expect few systematic differences in the dusty star clusters modelled with DUSTY relative to the Cloudy models. The metallicity of the input spectrum was taken to be $20 \%$ solar; this was to better account for possible lowermetallicity embedded sources in the outer disk. However, as illustrated below, this choice of metallicity does not significantly affect the resulting IRAC/MIPS-24 colours.

We show examples of the resulting DUSTY spectra in Fig. 11. Here we plot only 5 values of $A_{V}$, namely $0.1,2.15$, 10.0, 21.5, and 100 (shown as long-dashed, short-dashed, dotted, dot-dashed, and solid lines, respectively). Also shown (as a heavy solid line which ends at $\lambda \sim 100 \mu \mathrm{m}$ ) is the input stellar spectrum of a 3 Myr starburst from SB99. The figure clearly shows the importance of nebular continuum emission and the onset of warm dust in the spectral region sampled by IRAC. 


\subsubsection{Blackbodies}

To illustrate the similarity between the emission from stellar populations and blackbodies in the IRAC bands, we also calculated blackbody spectra at temperatures ranging from $100000 \mathrm{~K}$ to $300 \mathrm{~K}$. In the IRAC/MIPS spectral region, there is virtually no difference among the blackbodies from $100000 \mathrm{~K}$ to $3500 \mathrm{~K}$. As expected, their colours are also very similar to the SB99 simulations of the stellar populations in a 3 Myr cluster.

\subsubsection{Interstellar medium with $\mathrm{PAH}$ features}

An example of the spectrum of the diffuse ISM at high Galactic latitudes was also included in the diagnostics. The spectrum was taken from Fig. 8 of Li \& Draine (2001), and the digital version was kindly provided by B. Draine. In the IRAC/MIPS-24 spectral range, the diffuse high-Galactic latitude ISM shows extremely strong PAH emission, superimposed on a rising continuum.

\subsubsection{Reflection nebula NGC 7023}

NGC 7023 is a well-studied PDR that, because of its proximity, is an excellent source to study the physical processes occurring in such regions. It is a reflection nebula illuminated by a B3Ve star, and is located in a cavity of the molecular cloud. This cavity hosts a dense PDR with its peak located away from the star itself (Fuente et al. 2000). We obtained the short-wavelength portion of the ISO spectrum of this object from the uniform database published by Sloan et al. (2003). Like the diffuse ISM, NGC 7023 also shows relatively strong PAH features superimposed on a rising continuum.

\subsubsection{Results}

The IRAC and IRAC/MIPS-24 diagnostic diagrams relative to the models described above are shown in the bottom panels of Figs. 12-14. Blackbodies are depicted by filled black circles. NGC 7023 is represented by a (yellow) triangle, the diffuse highlatitude ISM by an inverted (cyan) triangle, the SB99 simulations by (grey) diamonds. The Cloudy HII region is represented by filled green squares. Finally, the DUSTY models are represented by filled $\left(T_{\text {in }}=300 \mathrm{~K}\right)$ and empty $\left(T_{\text {in }}=600 \mathrm{~K}\right)$ circles, for a variety of shell thickness $Y_{\text {out }}=10$ (solid), 100 (dashed), 1000 (dash-dot) and 10000 (dotted line).

There are several interesting features that can be noticed in the plots. First, a PAH-dominated spectrum such as the diffuse ISM, the small dense Cloudy HII region, and the reflection nebula have virtually identical IRAC colours to some of the $T_{\text {in }}=300 \mathrm{~K}$ DUSTY models. The implication here is that strong PAH features can be easily confused with a rising continuum in the MIR. The only way to resolve the ambiguity is through detailed spectral information or by using the $24 \mu \mathrm{m}$ filter in conjunction with the IRAC $8 \mu \mathrm{m}$ one. Indeed, our plots show that only 2 IRAC filters, together with MIPS-24, are sufficient to separate strong continuum emission in the MIR from strong PAH features. Second, Fig. 12 shows that strongly embedded sources (e.g., $A_{V} \sim 100$ ) occupy a well-defined region in the IRAC colour diagram. This may potentially be useful for singling out ultra-compact dusty HII regions from our $24 \mu \mathrm{m}$ source catalogue. Third, stars (roughly equivalent to $3500 \mathrm{~K}$ blackbodies) occupy a well-defined region in both diagrams, and should therefore be easily separable from other classes of objects.
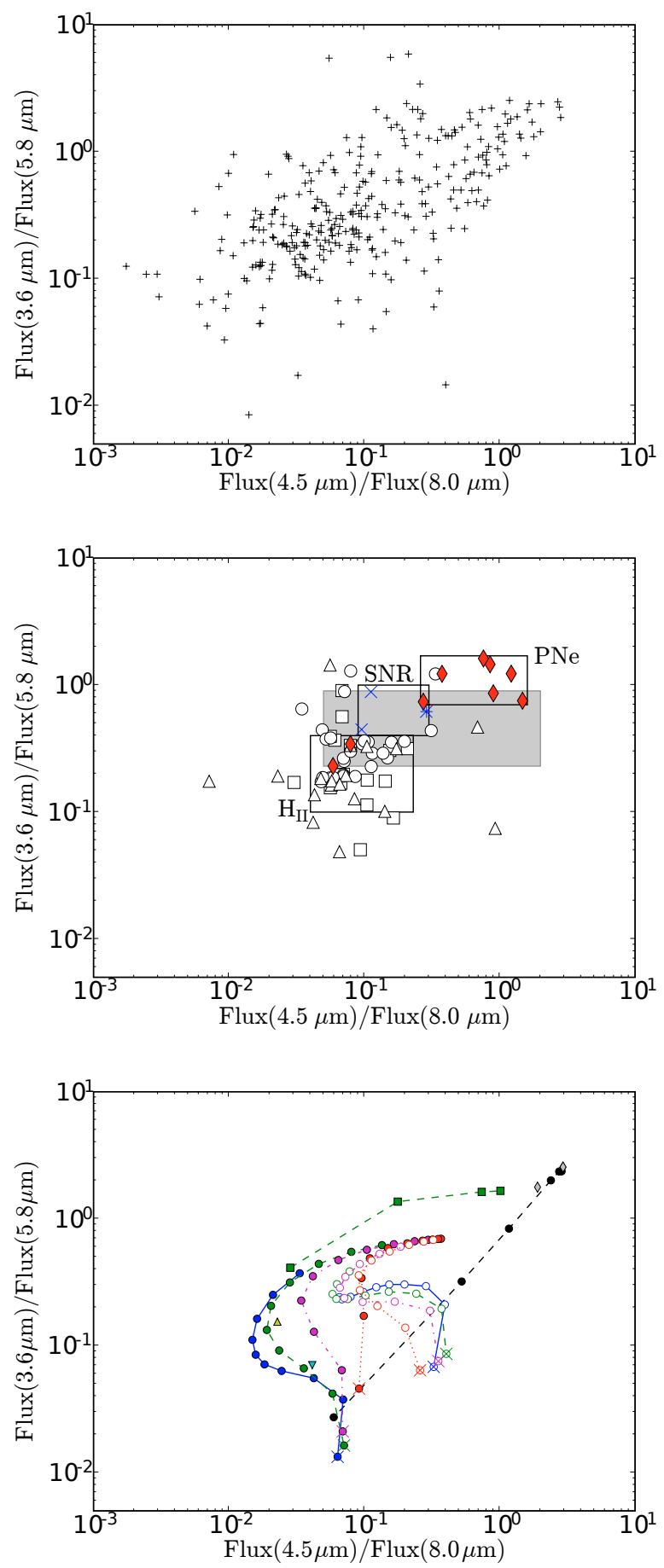

Fig. 12. Bottom panel: blackbodies are depicted by filled (black) circles. NGC 7023 is represented by a (yellow) triangle, the ISM by Draine by an inverted (cyan) triangle, the SB99 simulations by (grey) diamonds. The Cloudy HII region is represented by filled (green) squares. Finally, the DUSTY models are represented by filled $\left(T_{\text {in }}=300 \mathrm{~K}\right)$ and empty $\left(T_{\text {in }}=600 \mathrm{~K}\right)$ circles, for a variety of shell thickness $Y_{\text {out }}=10$ (solid), 100 (dashed), 1000 (dash-dot) and 10000 (dotted line). Different values of $A_{V}$ (logarithmically from 0.1 to $100 \mathrm{mag}$ ) for a given $T_{\text {in }}$ and $Y_{\text {out }}$ are connected by the different lines; the highest value of $A_{V}$ is marked by $\times$. Middle panel: the open symbols represent the HII regions selected in IR (squares), radio (triangles) and optical (circles). The (blue) crosses represent the SNRs optically selected and the (blue) plus the SNRs radio selected. The filled (red) diamonds represent the PNe. The open rectangles delineate the zones occupied by the majority of sources of a given type. The grey rectangle delimits the zone of molecular shocked SNRs (Reach et al. 2006). Top panel: the $24 \mu \mathrm{m}$ sources are depicted by (black) pluses. 

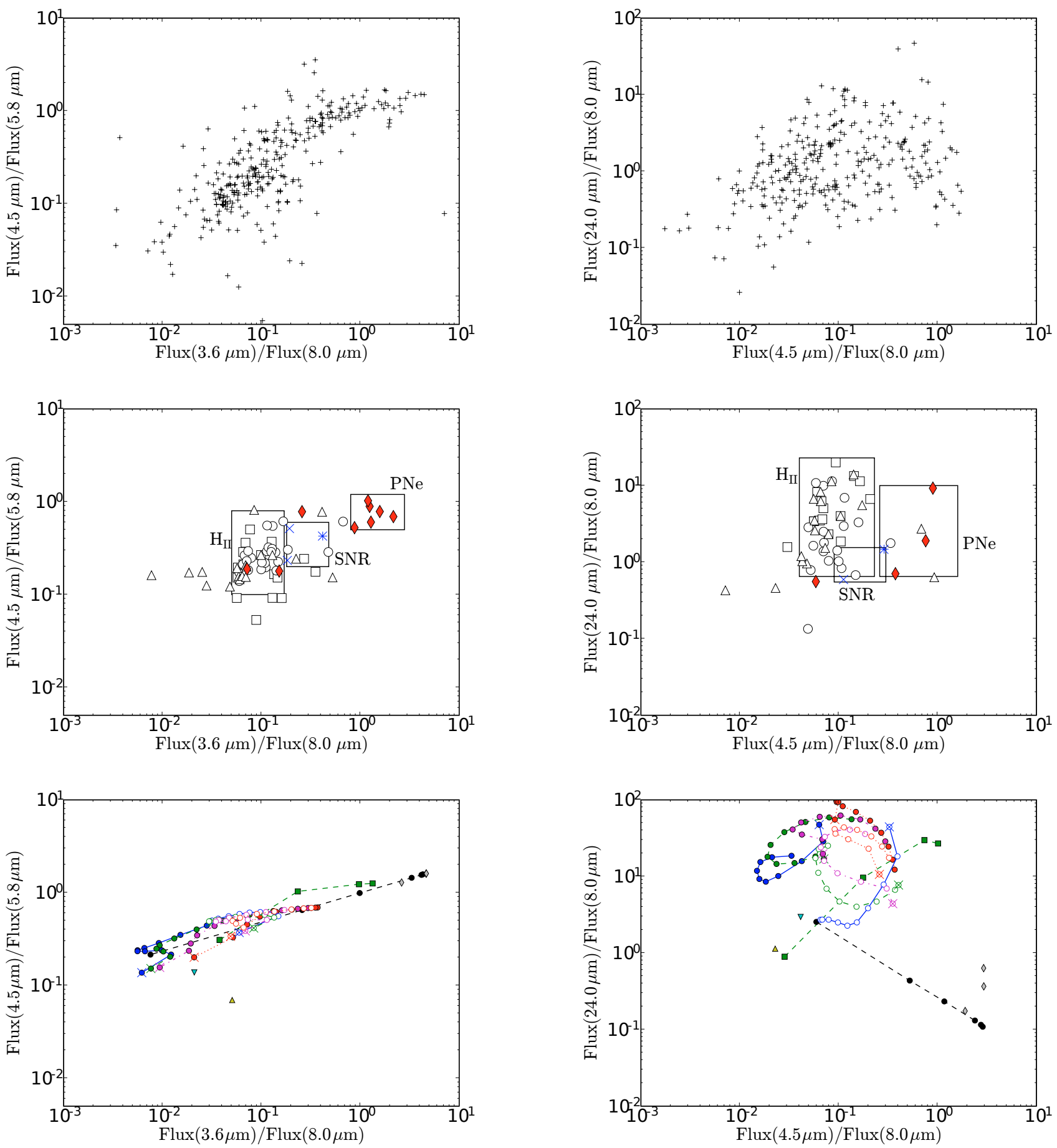

Fig. 13. Same as Fig. 12, but for different IRAC flux ratios.

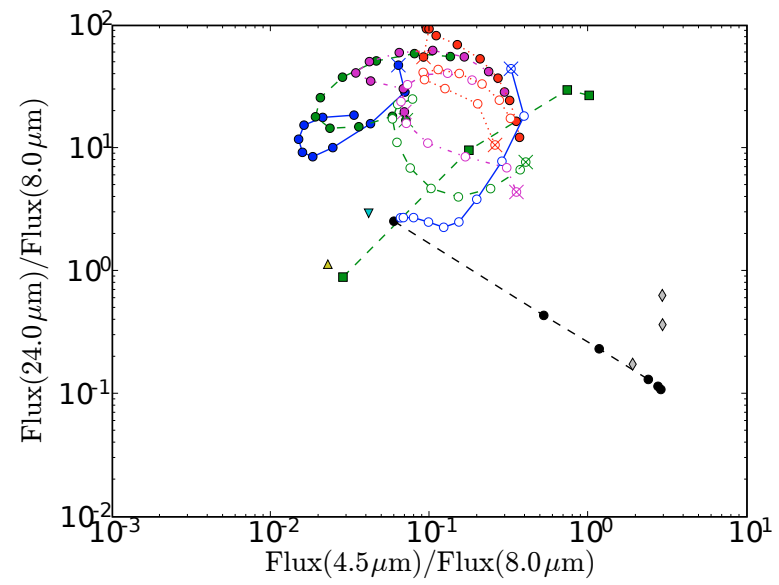

Fig. 14. Same as Fig. 12, but now including the MIPS $24 \mu \mathrm{m}$ flux ratio.

On the negative side, the DUSTY model predictions in the IRAC/MIPS-24 diagram are not easily distinguishable from Cloudy predictions. Moreover, even the highly embedded clusters do not occupy a distinct region in the colour diagrams. This makes the use of IRAC bands necessary, in order to better separate high extinction regions from optically thin ones.

\subsection{Observational diagnostic diagrams}

A comparison of the IRAC colours of the models+templates vs. observations of the different kinds of nebulae of Sect. 4 is shown in Figs. 12 and 13. Diagnostic diagrams using IRAC colours

were presented by Reach et al. (2006) (focusing on SNRs emission mechanisms) and by Cohen et al. (2007). We present two versions of the IRAC diagram, in order to better assess the diagnostic value of the different ratios. We also propose in Fig. 14 a new diagnostic diagram involving the $24 \mu \mathrm{m}$ MIPS channel which allows us to disentangle some degeneracies present when using only IRAC colours. In the next section, will use both the theoretical colour diagnostics and the observed colours of known-type objects to investigate the nature of sources selected at $24 \mu \mathrm{m}$. 


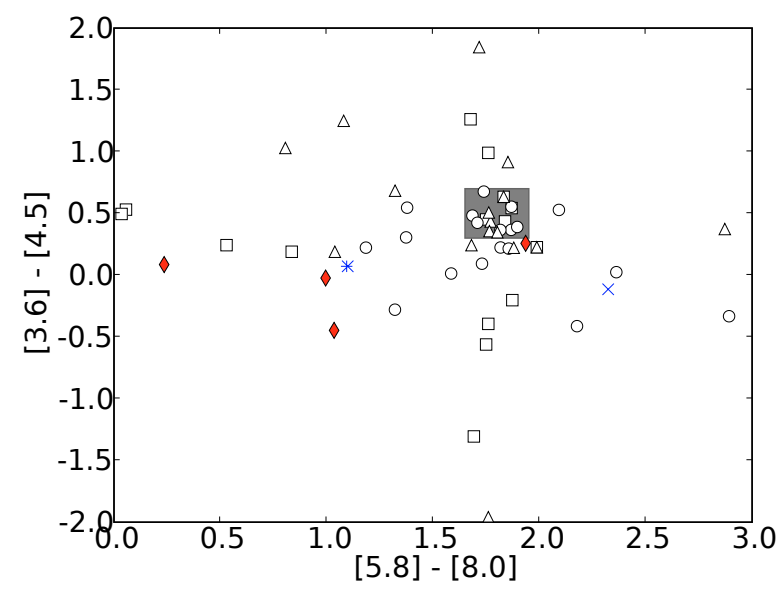

Fig. 15. Diagnostic diagrams in magnitude, for comparison with Cohen et al. (2006). The open symbols represent the HII regions selected in IR (squares), radio (triangles) and optical (circles). The (blue) crosses represent the SNRs optically selected and the (blue) plus the SNRs radio selected. The filled (red) diamonds represent the PNe. Two radio HII regions are lying outside the limits of the displayed plot.

\subsubsection{HII regions}

In the first IRAC colour diagram (see the middle panels of Figs. 12 and 13), we find a concentration of HII regions in the zone dominated by PAH emission (see Reach et al. 2006). In the same zone, lie the reflection nebulae NGC 7023 and the diffuse Galactic ISM as well. The dispersion in the colours of HII regions makes it difficult to separate them from SNRs and PNe. Statistically, the HII regions are more concentrated near the regions defined by low values of the two shortest IRAC channels and/or equivalently high values of the two longer IRAC wavelengths. The most intense PAH bands $(6.2,7.7,8.6 \mu \mathrm{m})$ are located in the 5.8 and $8.0 \mu \mathrm{m}$ channels and this may explain the colours of HII regions.

We compared the IRAC colours of our catalogued HII regions with the diffuse HII regions studied by Cohen et al. (2006). Upon conversion to their magnitude scale (Pahre et al. 2004), we find the IRAC-colour distributions to be mutually consistent.

\subsubsection{Supernovae remnants}

As a word of caution, since (especially young) SNRs are often associated with HII regions, they are often hard to discern and to measure photometrically given the crowding and confusion in the field. In the colour diagrams, the few detected SNRs lie between the HII regions and the PNe, generally closer to the HII regions.

Since the colours of pure synchrotron emission are never observed, there are several alternative emission mechanisms for the IR radiation that have been proposed by Reach et al. (2006) such as shocked molecular lines, shocked ionic lines, and PDR PAH emission. About half of their SNRs have colours consistent with molecular shocks, $17 \%$ are consistent with ionic shocks, and $22 \%$ with PAH emission from unshocked ISM. The others are thought to represent a mixture of shock types. All our 4 SNRs appear to belong to the class of shocked molecular emission (see the - grey - filled rectangle in the middle panel of Fig. 12), that is shocked molecular gas cooling mainly via MIR emission lines. Also, optically, SNRs appear as shock-heated emission nebulae (Gordon et al. 1999), confirming the prevalence of the two shock mechanisms described by Reach et al. (2006). The colours of our
SNRs are also close to the colours of some HII regions. Note that the colours of the SNRs could depend of the epoch at which the SNR is observed (for instance because of the onset or decrease of shocks as they expand into the ISM).

\subsubsection{Planetary nebulae}

With few exceptions, catalogued $\mathrm{PNe}$ are confined to a region characterised by low values of the 5.8 and $8.0 \mu \mathrm{m}$ emission, the bandpasses where PAHs are believed to contribute substantially to the IR emission. Indeed, PNe spectra usually show an absence or very weak presence of PAH, as noted by Bernard-Salas et al. (2002) with ISO and by Bernard-Salas et al. (2004) and with Bernard-Salas et al. (2006) Spitzer. In the $4.5 \mu \mathrm{m}$ band, the [Mg iv] $4.485 \mu \mathrm{m}$ and [A vi] $4.530 \mu \mathrm{m}$ lines could give an additional contribution for high-excitation $\mathrm{PNe}$ (Cohen et al. 2005). Thus, in absence of detailed spectral information Spitzer colours can provide some diagnostics to help to identify PNe candidates (see also Hora et al. 2004).

\section{The $24 \mu \mathrm{m}$ sources in $M 33$}

Spitzer images provide a unique opportunity to compile vast catalogues of MIR discrete sources down to stellar luminosities for galaxies in the Local Group. In the present section we describe and discuss some features of the catalogue of $24 \mu \mathrm{m}$ sources (24Ss) which we have generated for M33. In the first subsection, we describe the source selection criterion and flux measurements; in the second subsection, we investigate their nature in light of both theoretical and observational diagnostic diagrams; in the last subsection we present their luminosity function.

\section{1. $24 \mu \mathrm{m}$ source catalogue}

To select and measure individual sources from the $24 \mu \mathrm{m}$ image, we used the SExtractor software (Bertin \& Arnouts 1996). Using a convolution with a Gaussian filter we claim a detection when the source area is larger than 10 pixels and the peak signalto-noise ratio is at least 10 times the local background. Guided by the galaxy pattern traced by the $24 \mu \mathrm{m}$ diffuse emission, we removed spurious sources such as foreground stars or artifacts due to map edge effects. The final catalogue comprises $515 \mathrm{ob}-$ jects; the positions are listed in Table 3. The brightest sources at $24 \mu \mathrm{m}$ are located in the central region and along the spiral arms. Fainter sources are more smoothly distributed but still follow the flocculent pattern of the galaxy.

\subsection{Nature of the $24 \mu \mathrm{m}$ sources}

Perhaps not surprisingly, sources in our $24 \mu \mathrm{m}$ catalogue turn out to have a higher detection rate than any of the other kinds of objects considered in the previous sections. This is particularly marked at $5.8 \mu \mathrm{m}$ where the detection rate for the $24 \mu \mathrm{m}$ sample is much higher than for HII regions (see Fig. 7) although, as seen in Fig. 9, the averaged SED of the 24Ss is similar to the one of HII regions.

The position of the $24 \mathrm{Ss}$ in the colour diagrams are illustrated in the top panels of Figs. 12-14. In Fig. 12, the region with the highest concentration matches well the one of HII regions. However the distribution of $24 \mu \mathrm{m}$ sources extends into the regions populated by SNRs and PNe. The comparison with the theoretical models indicates a paucity of sources with high 
extinction. However, as Fig. 12 shows, neither a pure blackbody, Cloudy, or the two DUSTY models can alone reproduce the colour distribution of the $24 \mu \mathrm{m}$ sources. Figure 13 shows a clear departure of the 24Ss sample from the expected narrow 4.5/5.8 colour dispersion. Such departure is particularly marked in the red tail of the distribution where both 24Ss and knowntype sources tend to have colours similar to the PDR observed in the reflection nebulae NGC 7023 .

The hybrid IRAC-24 $\mu \mathrm{m}$ MIPS colour diagnostics of the selected sources are illustrated in Fig. 14. No $24 \mathrm{~S}$ displays a $24 / 8.0 \mu \mathrm{m}$ flux ratio as high as the DUSTY models with shell inner temperature $T_{\text {in }}=300 \mathrm{~K}$; the warmer models $\left(T_{\text {in }}=600 \mathrm{~K}\right)$ seem more appropriate to describe their physical conditions. The range spanned by the $24 / 8.0$ ratio in the $24 \mu \mathrm{m}$ sources is large but similar to the one covered by the catalogued HII regions, indicating that in both samples the contribution from PAH features may vary widely. So, $24 \mathrm{Ss}$ with both a low $4.5 / 8.0$ and a low 24/8.0 ratio could well be HII regions with high PAH emission (see, for instance, the location of the radio-selected HII regions). The Cloudy HII region simulations seem to match well the colours of the $24 \mathrm{Ss}$, in spite of their large scatter. On the contrary, the observations rule out most stellar colours: both pure blackbodies and the SB99 templates. However, as above, the general agreement between any single model and the 24Ss distribution in the colour diagrams is unsatisfactory; such a trend is probably due to a variable $\mathrm{PAH}$ component which is not considered in most of our templates (but see loci of NGC 7023 and the ISM). In the next section, we will select a subsample of $24 \mathrm{Ss}$ associated with the $\mathrm{H} \alpha$ emission. These sources, likely to be optically visible HII regions, achieve a better agreement with lowextinction models.

\subsection{Luminosity function of $24 \mu \mathrm{m}$ sources}

The $24 \mu \mathrm{m}$ emission of M 33 within an ellipse of major axis $40^{\prime}$ and axial ratio of 0.62 is $40.9 \mathrm{Jy}$. At a distance $D=840 \mathrm{kpc}$, such ellipse corresponds to a circle at galactocentric distance of $4.8 \mathrm{kpc}$ inclined by $52^{\circ}$ with respect to our line of sight. The $24 \mu \mathrm{m}$ integrated emission is in good agreement with the value reported by Hinz et al. (2004) (see their Fig. 2) and implies a $24 \mu \mathrm{m}$ luminosity $\left(L=v F_{v} \times 4 \pi D^{2}\right)$ of $4.3 \times 10^{41} \mathrm{erg} \mathrm{s}^{-1}$. The contribution of the 515 discrete 24 Ss amounts to $1.4 \times$ $10^{41} \mathrm{erg} \mathrm{s}^{-1}$, hence the diffuse component accounts for two thirds of the $24 \mu \mathrm{m}$ emission.

The cumulative luminosity function of the 515 discrete sources catalogued at $24 \mu \mathrm{m}$ is shown in Fig. 16. In this figure, the abscissas are reported as flux density at $24 \mu \mathrm{m}$ in mJy and also as total IR luminosity (TIR). To estimate the TIR luminosity, we follow the prescription of Calzetti et al. (2005) (see their Eq. (1)). The conversion involves the $24 \mu \mathrm{m}$ and the $8 \mu \mathrm{m}$ measurements. Since we do not have a detection at $8 \mu \mathrm{m}$ for all $24 \mathrm{Ss}$, we compute the average $8 \mu \mathrm{m} / 24 \mu \mathrm{m}$ flux ratio for the sources detected in both bands: the average is close to unity and therefore, in the lower panel of Fig. 16, which includes all 24Ss, we use $\log L($ TIR $)=\log L(24 \mu \mathrm{m})+0.908$. As a check, in the upper panel of Fig. 16, we plot the cumulative luminosity function only for the sources detected in both bands, using for each source the measured $8 \mu \mathrm{m} / 24 \mu \mathrm{m}$ flux ratio: the shape and meaning (see below) of the two curves is quite compatible. The main effect, when using a constant average flux ratio, is a slight expansion of the range in $L$ (TIR) by about 0.4 dex, together with a brightening by the same factor.

From the flattening of the faint end of the distribution, we estimate the completeness limit of the catalogue around $1 \mathrm{mJy}$, or an $L(\mathrm{TIR})$ of $5 \times 10^{37} \mathrm{erg} \mathrm{s}^{-1}$ on the averaged scale. That is the bolometric luminosity of a B1.5 V star (Cox 2000) and so our listing should be quite complete even for faint obscured HII regions. Apart from the levelling off at low fluxes, as often observed for HII regions, open clusters, and associations (McKee \& Williams 1997), the luminosity function displays a double slope behaviour, markedly steeper at the high luminosity tail. The change of slope, in the simplest scenarios, represents the change of regime between poor and rich clusters, where rich means numerous enough to represent fairly the high-mass IMF (Oey \& Clarke 1998). In this framework, the transition point between the two regimes marks the luminosity of the single brightest star; below this value, the observed statistics is modified by the sampling variance. We find that the transition point occurs around $F_{\nu}(24 \mu \mathrm{m})=70 \mathrm{mJy}$, that is, in the averaged scale, an $L(\mathrm{TIR}) \simeq 5 \times 10^{39} \mathrm{erg} \mathrm{s}^{-1}$ which, still bolometrically, corresponds to an O3 V star (Vacca et al. 1996). This implies: first, that most of the bright $24 \mathrm{Ss}$ are in fact luminous young stellar clusters and, second, that our $L$ (TIR) scale cannot be grossly mistaken. From $F_{v}(24 \mu \mathrm{m})=1$ to $F_{v}(24 \mu \mathrm{m})=70 \mathrm{mJy}$ we fit a power index $-0.55 \pm 0.01$ (plain - red - line) and, for larger values an index $-1.09 \pm 0.08$ (dotted - green - line). It is easily shown that an index about unity, in the log-log cumulative distribution, implies for the rich galactic clusters a distribution $N(L) \mathrm{d} L \propto L^{-2} \mathrm{~d} L$ or, equivalently, $N\left(n_{*}\right) \mathrm{d} n_{*} \propto n_{*}^{-2} \mathrm{~d} n_{*}, n_{*}$ being the number of stellar members. Again, this is precisely the slope observed in the luminosity function of HII regions in late spirals (Caldwell et al. 1991), as well as the slope determined for Galactic clusters and associations, e.g. McKee \& Williams (1997).

The brightest source reported in Fig. 16, at $F_{v}(24 \mu \mathrm{m}) \simeq$ $1.8 \mathrm{Jy}$, would be roughly equivalent to an open cluster with around $3.5 \times 10^{7} L_{\odot}$; assuming that all members $\left(0.06\right.$ to $\left.90 M_{\odot}\right)$ are on the main sequence, this makes a cluster of some $10^{5} M_{\odot}$, more than $10^{6}$ members, and more than 1000 ionising $\left(>15 M_{\odot}\right)$ stars. On the other side, the faintest detected discrete 24Ss have $F_{v}(24 \mu \mathrm{m})$ of $0.1-0.2 \mathrm{mJy}$, that is $L(\mathrm{TIR}) \simeq 10^{37} \mathrm{erg} \mathrm{s}^{-1}$. This is about the bolometric luminosity of zero age main sequence B2 stars (Panagia 1973); so it is possible that these objects are also (the faintest) HII regions and our luminosity distribution appears to precisely match the whole range expected for ionised regions and their complexes. Anyway at low luminosity, especially below $10^{4} L_{\odot}$, the situation may be more intricate since also evolved stars can contribute substantially, more or less depending on the history of the region, to the population of IR sources; carbon giants, for example, at the distance of M 33 are expected to emit an average $F_{v}(24 \mu \mathrm{m}) \simeq 0.25 \mathrm{mJy}$ (Groenewegen et al. 2007). The nature of the 24Ss will be further explored, by investigating their spatial location within the galaxy and IR colours, in a forthcoming paper.

\section{Star formation}

\subsection{Global star formation rates}

In regions of moderate visual extinction, $\mathrm{H} \alpha$ line emission is expected to be a reliable indicator of recent star formation. M 33 has globally a low extinction rate and therefore we can assess the performance of MIR emission as a star formation tracer, by comparing the SFR inferred from MIR emission with that derived from $\mathrm{H} \alpha$ emission.

$\mathrm{H} \alpha$ fluxes were measured on the map of Hoopes \& Walterbos (2000) cited earlier (Sect. 2.3) and converted to SFR following Kennicutt (1998). We find a total SFR from $\mathrm{H} \alpha$, within $5 \mathrm{kpc}$ 


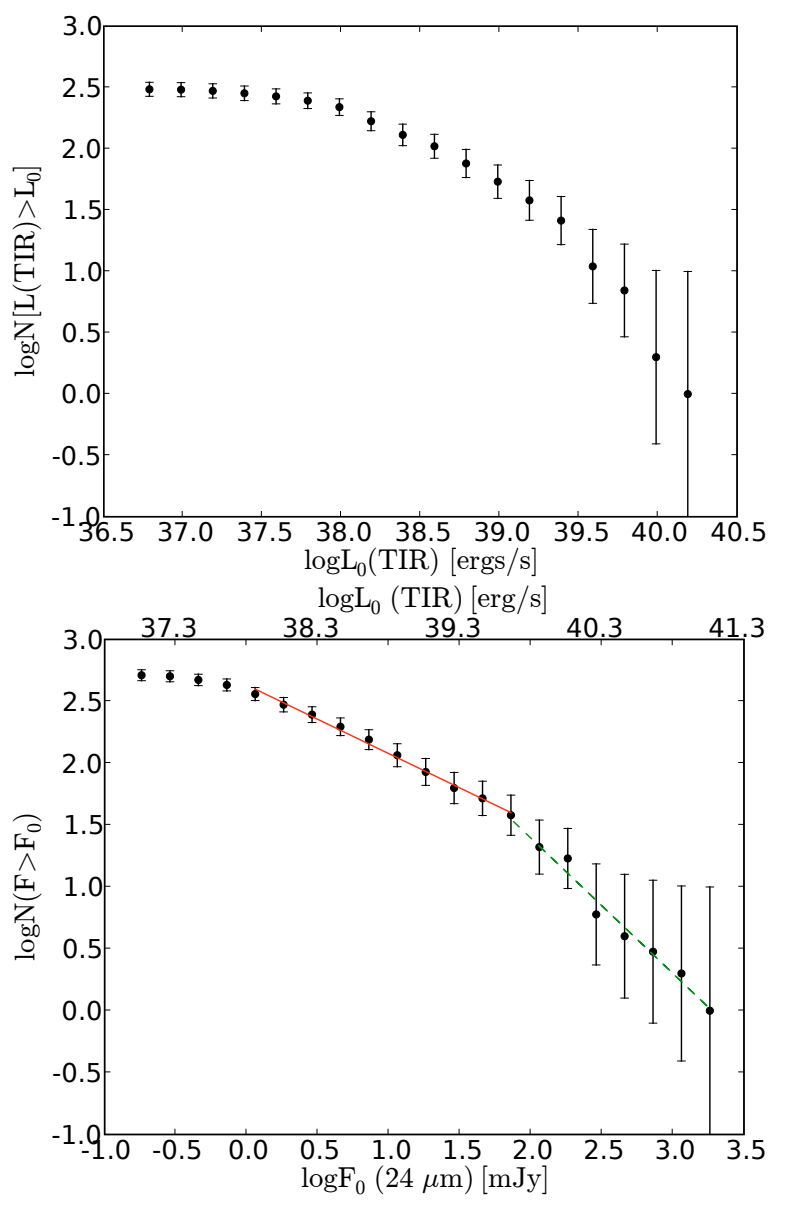

Fig. 16. Bottom panel: cumulative distribution of the $24 \mu \mathrm{m}$ sources. The $24 \mu \mathrm{m}$ fluxes are reported $F_{v}(24 \mu \mathrm{m})$ (bottom axis), as well as the TIR luminosity $L_{v}$ (TIR) (top axis). The linear best fits are depicted by a (red) solid line for the $F_{v}(24 \mu \mathrm{m})$ fluxes between 1 and $70 \mathrm{mJy}$, and by a (green) dashed line for the $F_{v}(24 \mu \mathrm{m})$ fluxes greater than $70 \mathrm{mJy}$. Top panel: cumulative distribution of the $24 \mu \mathrm{m}$ sources detected also at $8.0 \mu \mathrm{m}$, the TIR luminosity is calculated using for each source the effective $8 \mu \mathrm{m} / 24 \mu \mathrm{m}$ flux ratio.

from the centre, of $0.22 M_{\odot} \mathrm{yr}^{-1}$ if no extinction corrections are taken into account. On average, this is a reasonable assumption since Tabatabaei et al. (2007b) found a perfect $3.6 \mathrm{~cm}-\mathrm{H} \alpha$ correlation for the thermally emitting regions in M 33. In any case, given that the diffuse $\mathrm{H} \alpha$ emission amounts to $40 \%$ of the total (Hoopes \& Walterbos 2000), an extinction correction of individual sources by $A_{V}=1$ on average (as claimed by Devereux et al. 1997 and Israel \& Kennicutt 1980), would increase the SFR in the same area to $0.3 M_{\odot} \mathrm{yr}^{-1}$.

To estimate the SFR from the IR emission, we used two independent methods: we first estimate the TIR luminosity within a radius of $5 \mathrm{kpc}$ using the data at 8.0 and $24 \mu \mathrm{m}$. With a flux of $47.3 \mathrm{Jy}$, the integrated $8.0 \mu \mathrm{m}$ luminosity $L_{v}(8.0 \mu \mathrm{m})$ is $4.0 \times 10^{28} \mathrm{erg} \mathrm{s}^{-1} \mathrm{~Hz}^{-1}$, corresponding to $v L_{v}(8)=1.5 \times$ $10^{42} \mathrm{erg} \mathrm{s}^{-1}$. This is slightly higher than $L_{v}(24 \mu \mathrm{m})=3.5 \times$ $10^{28} \mathrm{erg} \mathrm{s}^{-1} \mathrm{~Hz}^{-1}$ (or $\left.v L_{v}(24)=4.4 \times 10^{41} \mathrm{erg} \mathrm{s}^{-1}\right)$. Such a difference in the 8 and $24 \mu \mathrm{m}$ flux ( $47.3 \mathrm{vs}$. $40.9 \mathrm{Jy})$ is consistent with the IR SEDs measured in nearby galaxies (Dale et al. 2005), in which IR fluxes at $8.0 \mu \mathrm{m}$ often exceed those at $24 \mu \mathrm{m}$. Using these values, Eq. (1) in Calzetti et al. (2005) yields an $L(T I R)=$ $1.0 \times 10^{9} L_{\odot}$. The IR SFR is usually computed from the FIR (40-120 $\mu \mathrm{m})$ luminosity. Calzetti et al. (2000) found that the ratio $L_{\mathrm{IR}}(1-1000 \mu \mathrm{m}) / L_{\mathrm{IR}}(40-120 \mu \mathrm{m})$ is about 1.75 . In our case this translates into a FIR luminosity of $5.8 \times 10^{8} L_{\odot}$ within a radius of $5 \mathrm{kpc}$ in M33. This is in good agreement with the value of $6.5 \times 10^{8} L_{\odot}$ found from the total IRAS fluxes reported by Rice et al. (1990), especially considering that we are including only the central $10 \mathrm{kpc}$ in our estimate. Following the precepts of Buat \& Xu (1996) for late spirals, that is $\operatorname{SFR}\left[M_{\odot} \mathrm{yr}^{-1}\right]=8 \times$ $10^{-44} L$ (FIR) with uncertainties of around $50 \%$, we obtain a SFR equal to $0.18_{-0.07}^{+0.18} M_{\odot} \mathrm{yr}^{-1}$. Within the uncertainties this estimate is compatible with those derived from $\mathrm{H} \alpha$ emission (with and without extinction corrections). We obtain the same value from the TIR-SFR relation by Kennicutt (1998), taking into account the luminosity conversion (TIR vs. FIR).

The second method to derive the SFR from the IR emission involves the linear relation between radio and IR luminosities, and SFR (Yun et al. 2001). Wu et al. (2005) derived SFR directly from the $24 \mu \mathrm{m}$ luminosity (see their Eq. (1)): in our case, this translates into a SFR of $0.17 M_{\odot} \mathrm{yr}^{-1}$. Using the $8 \mu \mathrm{m}$ luminosity (their Eq. (2)) gives a higher value of SFR, $0.26 M_{\odot} \mathrm{yr}^{-1}$, even after subtracting the stellar contribution to the $8 \mu \mathrm{m}$ flux, $\sim 10 \%$. Although within the errors of the $24 \mu \mathrm{m}$ and FIR results (from Rice et al. 1990), the $8 \mu \mathrm{m}$ value is $\lesssim 40 \%$ higher than either the $24 \mu \mathrm{m}$ or the $\mathrm{H} \alpha$ one. A similar trend was found by Calzetti et al. (2005) in which the $8 \mu \mathrm{m}$ SFR estimate can be significantly larger in weakly ionized regions such as the disk of M 33 taken globally. This is because the $8 \mu \mathrm{m}$ emission is produced by more than one mechanism, and thus does not trace SFR as accurately as other IR wavelengths.

In general then both MIR prescriptions for computing the SFR reach a satisfactory agreement with the $\mathrm{H} \alpha$-derived value and, beyond confirming the adopted SFR computing scheme, implies: a) that actively star forming sites are the main contributors to the MIR emission; b) that mean extinction is low also in the inner HII regions; c) that highly obscured sources do not contribute significantly to the MIR emission.

\subsection{Star formation rates in $24 \mu \mathrm{m}$ sources}

We then searched for $\mathrm{H} \alpha$ emission at the location of the 24Ss. This was done in order to compare locally the SFRs from $\mathrm{H} \alpha$ with those from the MIR, but also to check whether sources with and without an $\mathrm{H} \alpha$ counterpart have on average the same IR colours. On the narrow-band (continuum subtracted) $\mathrm{H} \alpha$ image, we performed the photometry of the 24Ss using the same technique described in Sect. 4.1. We find that 293 of the 24Ss are also detected in $\mathrm{H} \alpha$ (within 3.' 6 ). An inspection of the $\mathrm{H} \alpha$ image shows that most bright $24 \mathrm{Ss}$ with no $\mathrm{H} \alpha$ counterpart actually lie near an HII region, with an offset which, though larger than the photometric errors, could still be accommodated in case of asymmetries of the $\mathrm{H} \alpha$ shell. This is the case, for example, of some sources along the spiral arms. Some faint $24 \mathrm{Ss}$ sources might instead really be absent in the $\mathrm{H} \alpha$ map. Indeed, the percentage of $24 \mathrm{Ss}$ undetected in $\mathrm{H} \alpha$ gets higher close to the completeness limit of our survey. We will analyse this finding in more detail in a forthcoming paper. We note however that the luminosity function of the 24Ss with $\mathrm{H} \alpha$ counterpart is totally compatible with the one for the whole sample presented in Sect. 6.3. This is because contamination of the $24 \mu \mathrm{m}$ sample by non star forming regions happens mostly below the completeness limit of the derived luminosity function.

To estimate the SFR in discrete sources, we adopted the same conversion methods used for the global SFRs and, in order to estimate the TIR luminosity following Calzetti's formula, we restrict our sample of 293 sources to the 186 sources which are also detected in the IRAC bands. The resulting SFRs are compared in 


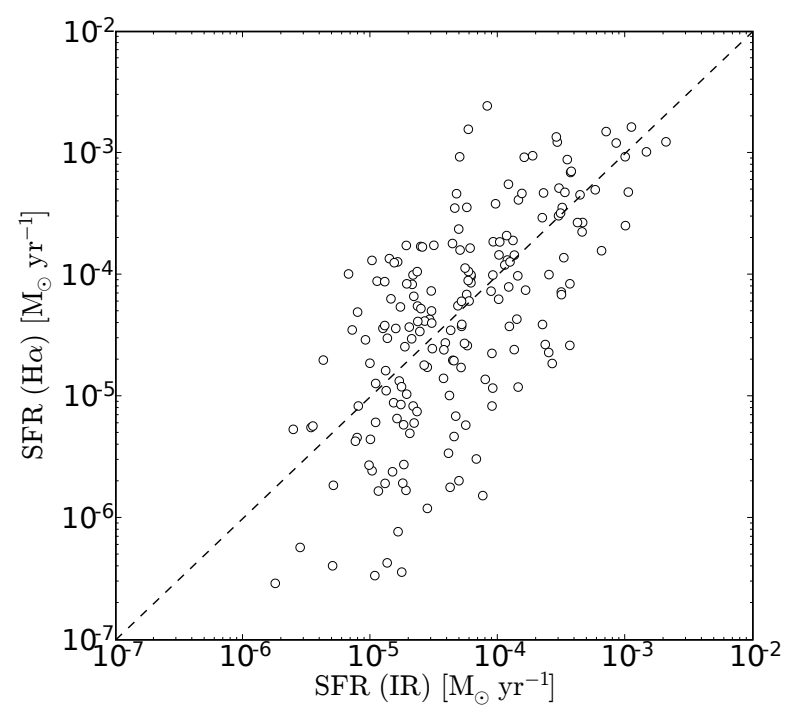

Fig. 17. Comparison of the SFR calculated from the $\mathrm{H} \alpha$ luminosity and from the IR emission based on the 8.0 and $24 \mu \mathrm{m}$ emissions. The dashed line shows the $\mathrm{SFR}(\mathrm{H} \alpha)=\mathrm{SFR}(\mathrm{IR})$ relation.

Fig. 17, where the SFR is derived from $\mathrm{H} \alpha$ without any average or individual extinction corrections and the FIR fluxes have been converted to SFR as in the previous subsection: SFR $\left[M_{\odot} \mathrm{yr}^{-1}\right]=$ $8 \times 10^{44} L$ (FIR). The scatter about the line of slope unity is large, roughly a factor of $5(1 \sigma)$, and it is difficult to assess the presence of any systematic deviation. The scatter itself, given its magnitude, cannot be imputed to extinction only. Most of the variance is likely linked to the uncertainties intrinsic to the methods used to infer SFRs. A very similar plot can be obtained using the SFR estimated only from the $24 \mu \mathrm{m}$ emission (see previous subsection) of the discrete sources and is consequently not shown here.

We can compare the IR colours of the latter sample with those of the complementary sample made up by the $12124 \mathrm{Ss}$ with no $\mathrm{H} \alpha$ counterpart, selecting sources detected in all IRAC and MIPS-24 bands. One such diagram is in Fig. 18 and should be compared with the equivalent diagnostic diagram of Sect. 5 . The 24Ss without $\mathrm{H} \alpha$ actually seem to be less dusty sources than those emitting also in $\mathrm{H} \alpha$, a trend confirming that 24Ss with no $\mathrm{H} \alpha$ are not more embedded into dust.

\section{Summary and conclusions}

This is the first in a series of papers aiming to investigate the star formation history in M 33 by means of the IR data from Spitzer. We retrieved and reduced IRAC and MIPS data producing maps roughly the size of the entire star forming galaxy disk. These were used to study the nature of the MIR emission, both globally (large scale structure of dust emission) and locally (by means of discrete sources). Our main results are the following:

- On large scales, the 3.6/4.5 and 5.8/8.0 ratios are remarkably constant. The 3.6 and $4.5 \mu \mathrm{m}$ emission mainly arises from point sources while the 5.8 and $8.0 \mu \mathrm{m}$ bands present more diffuse emission following the morphological pattern of the galaxy (spiral arms). The 8.0/24 ratio is also rather constant suggesting a tight link between PAH and VSG properties. On the other hand, the 3.6/5.8 and 4.5/8.0 ratios reveal the flocculent spiral structure in M 33, because of the increasing dominance of the ISM toward longer wavelengths. The distribution of cooler dust is investigated via the MIPS images: the 24 and $70 \mu \mathrm{m}$ emissions match closely, while the $160 \mu \mathrm{m}$

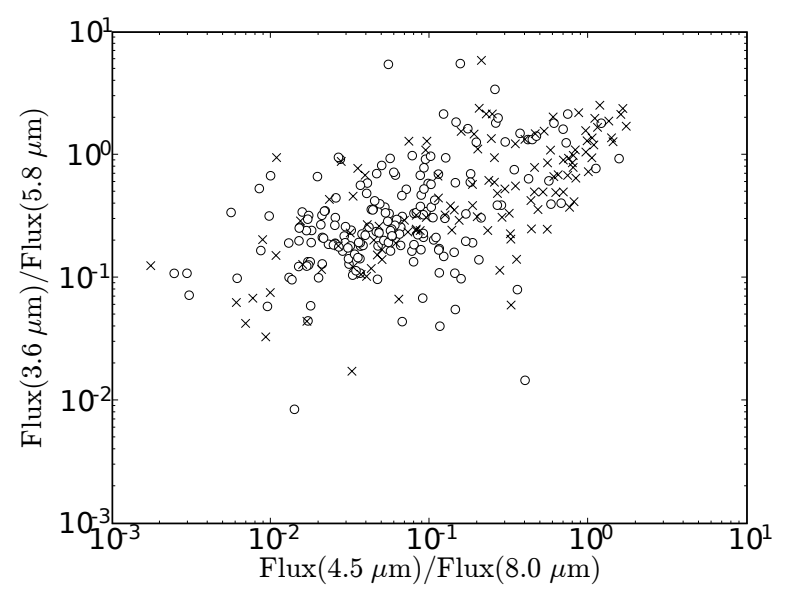

Fig. 18. Diagnostic diagram involving IRAC colours for the $24 \mu \mathrm{m}$ sources having a $\mathrm{H} \alpha$ counterpart (open circles) and for the $24 \mu \mathrm{m}$ sources with no $\mathrm{H} \alpha$ counterpart (crosses).

band reveals a more diffuse character in the cold-dust emission. The 24 and $70 \mu \mathrm{m}$ are also more tightly correlated with the $\mathrm{H} \alpha$ emission.

- In the four IRAC and $24 \mu \mathrm{m}$ MIPS bands, we performed photometry of catalogued discrete sources of various type, including HII regions, SNRs and PNe. We present the detection rates in each band for the various types of object, together with their IR SEDs. For all types of objects, a drop at $4.5 \mu \mathrm{m}$ is observed, likely due to the contribution of emission features or to the decreasing contribution of photospheric emission from the central star.

- The IR colours of HII regions, SNRs and PNe are used to define diagnostic diagrams. We observe a continuity among the various types: the HII regions appear more dusty than the PNe, which behave more like stellar objects. We also compared these colours with colours predicted by models (such as Cloudy, DUSTY, SB99, pure blackbody emission). The diagnostics based on IRAC alone cannot disentangle the different theoretical models, but usage of hybrid IRAC $+24 \mu \mathrm{m}$ colours allows the removal of some of the degeneracies. HII regions are better modelled by pure Cloudy HII regions (including PAHs) and by DUSTY models with inner-shell temperatures around $600 \mathrm{~K}$ and low extinction.

- We have compared the colours of some of the known-type objects in M 33 with similar objects in our Galaxy (HII regions) or in the LMC (SNRs) and, although with some scatter, we found good agreement for the location of the sources in the colour diagrams.

- From our $24 \mu \mathrm{m}$ image, we extracted a blind catalogue of 515 discrete sources, on which we performed photometry in the IRAC, and $24 \mu \mathrm{m}$ MIPS bands and in $\mathrm{H} \alpha$. Their colours match closely those of known-type sources, and in particular those of HII regions. Consequently, the models which better describe the nature of the $24 \mu \mathrm{m}$ sources are also pure Cloudy HII regions and low-extinction warm $\left(T_{\text {in }}=600 \mathrm{~K}\right) D U S T Y$ models.

- The total $24 \mu \mathrm{m}$ flux of the 515 discrete sources amounts to $1.4 \times 10^{41} \mathrm{erg} \mathrm{s}^{-1}$ while the diffuse emission, that is the residual flux, amounts to twice this value.

- We present the IR luminosity function of our $24 \mu \mathrm{m}$ sources, as a function of the $24 \mu \mathrm{m}$ flux and as well as a function of the total IR luminosity (estimated from the 8.0 and $24 \mu \mathrm{m}$ bands). The luminosity ranges from values typical of the faintest ionising stars to those of very large star formation 
complexes. The shape (double-sloped) is the same as that observed for Galactic HII regions and star complexes, though AGB stars could contribute significantly to the faintest bins.

- Within a radius of $5 \mathrm{kpc}$ in M33, we estimate a total IR luminosity of $1.0 \times 10^{9} L_{\odot}$ and the FIR luminosity is about $5.8 \times 10^{8} L_{\odot}$. This translates into an estimate of the SFR of $0.2 M_{\odot} \mathrm{yr}^{-1}$, in very good agreement with the SFR inferred from $\mathrm{H} \alpha$ emission.

- The relation between $24 \mu \mathrm{m}$ emission and HII regions is also investigated by searching directly for $\mathrm{H} \alpha$ counterparts of the $24 \mu \mathrm{m}$ sources. About half of the $24 \mu \mathrm{m}$ sources are very closely associated with $\mathrm{H} \alpha$ while the remaining ones are more distant from an $\mathrm{H} \alpha$ knot than the allowance by positional uncertainties. The SFR for the single sources in IR and $\mathrm{H} \alpha$ are consistent but the scatter is rather large and not imputable to extinction alone.

- The IR colours of the $24 \mu \mathrm{m}$ sources with and without $\mathrm{H} \alpha$ counterparts are quite similar. If anything, upon comparison with the theoretical diagnostics, those with $\mathrm{H} \alpha$ appear more dusty than the others.

Acknowledgements. We would like to thank Rene Walterbos for providing us the $\mathrm{H} \alpha$ image of M33, Laura Magrini for the complete list of optical HII regions, and Bruce Draine for the digital version of a diffuse ISM spectrum. The work of S. V. is supported by a INAF-Osservatorio Astrofisico di Arcetri fellowship. The Spitzer Space Telescope is operated by the Jet Propulsion Laboratory, California Institute of Technology, under contract with the National Aeronautics and Space Administration. This research has made use of the NASA / IPAC Extragalactic Database, which is operated by JPL/Caltech, under contract with NASA.

\section{References}

Allamandola, L. J., Tielens, G. G. M., \& Barker, J. R. 1989, ApJS, 71, 733 Arendt, R. G. 1989, ApJS, 70, 181

Bernard-Salas, J., Pottasch, S. R., Feibelman, W. A., \& Wesselius, P. R. 2002, A\&A, 387, 301

Bernard-Salas, J., Houck, J. R., Morris, P. W., et al. 2004, ApJS, 154, 271

Bernard-Salas, J., Peeters, E., Sloan, G. C., et al. 2006, ApJ, 652, L29

Bertin, E., \& Arnouts, S. 1996, A\&AS, 117, 393

Borkowski, K. J., Williams, B. J., Reynolds, S. P., et al. 2006, ApJ, 642, L141

Buat, V., \& Xu, C. 1996, A\&A, 306, 61

Caldwell, N., Kennicutt, R., Phillips, A. C., \& Schommer, R. A. 1991, ApJ, 370, 526

Calzetti, D., Kinney, A. L., Ford, H., Doggett, J., \& Long, K. S. 1995, AJ, 110, 2739

Calzetti, D., Armus, L., Bohlin, R. C., et al. 2000, ApJ, 533, 682

Calzetti, D., Kennicutt, Jr., R. C., Bianchi, L., et al. 2005, ApJ, 633, 871

Churchwell, E., Whitney, B. A., Babler, B. L., et al. 2004, ApJS, 154, 322

Ciardullo, R., Durrell, P. R., Laychak, M. B., et al. 2004, ApJ, 614, 167

Cohen, M., \& Barlow, M. J. 2005, MNRAS, 362, 1199

Cohen, M., Green, A. J., Roberts, M. S. E., et al. 2005, ApJ, 627, 446

Cohen, M., Green, A. J., Meade, M. R., et al. 2006, ArXiv Astrophysics e-prints

Cohen, M., Green, A. J., Meade, M. R., et al. 2007, MNRAS, 374, 979

Conti, P. S., \& Crowther, P. A. 2004, MNRAS, 355, 899

Corbelli, E. 2003, MNRAS, 342, 199

Corbelli, E., \& Salucci, P. 2000, MNRAS, 311, 441

Cox, A. N. 2000, Allen's astrophysical quantities, 4th edn. (New York: AIP Press; Springer), ed. A. N. Cox

Dale, D. A., Bendo, G. J., Engelbracht, C. W., et al. 2005, ApJ, 633, 857

Desert, F.-X., Boulanger, F., \& Puget, J. L. 1990, A\&A, 237, 215

Devereux, N., Duric, N., \& Scowen, P. A. 1997, AJ, 113, 236

Dopita, M. A., Fischera, J., Crowley, O., et al. 2006, ApJ, 639, 788

Draine, B. T. 2003, ARA\&A, 41, 241

Draine, B. T., \& Lee, H. M. 1984, ApJ, 285, 89

Duric, N., Gordon, S. M., Goss, W. M., Viallefond, F., \& Lacey, C. 1995, ApJ, 445,173

Engargiola, G., Plambeck, R. L., Rosolowsky, E., \& Blitz, L. 2003, ApJS, 149, 343
Fazio, G. G., Hora, J. L., Allen, L. E., et al. 2004, ApJS, 154, 10

Ferland, G. J. 2003, ARA\&A, 41, 517

Ferland, G. J., Korista, K. T., Verner, D. A., et al. 1998, PASP, 110, 76

Freedman, W. L., Wilson, C. D., \& Madore, B. F. 1991, ApJ, 372, 455

Fuente, A., Martin-Pintado, J., Rodriguez-Fernández, N. J., Cernicharo, J., \& Gerin, M. 2000, A\&A, 354, 1053

Garay, G., \& Lizano, S. 1999, PASP, 111, 1049

Gerardy, C. L., Meikle, W. P. S., Kotak, R., et al. 2007, ArXiv Astrophysics e-prints

Giveon, U., Sternberg, A., Lutz, D., Feuchtgruber, H., \& Pauldrach, A. W. A. 2002, ApJ, 566, 880

Giveon, U., Richter, M. J., Becker, R. H., \& White, R. L. 2007, AJ, 133, 639

Gordon, S. M., Kirshner, R. P., Long, K. S., et al. 1998, ApJS, 117, 89

Gordon, S. M., Duric, N., Kirshner, R. P., Goss, W. M., \& Viallefond, F. 1999, ApJS, 120, 247

Gorjian, V., Werner, M. W., Mould, J. R., et al. 2004, ApJS, 154, 275

Groenewegen, M. A. T., Wood, P. R., Sloan, G. C., et al. 2007, MNRAS, 376, 313

Helou, G., Roussel, H., Appleton, P., et al. 2004, ApJS, 154, 253

Heyer, M. H., Corbelli, E., Schneider, S. E., \& Young, J. S. 2004, ApJ, 602, 723

Hinz, J. L., Rieke, G. H., Gordon, K. D., et al. 2004, ApJS, 154, 259

Hippelein, H., Haas, M., Tuffs, R. J., et al. 2003, A\&A, 407, 137

Hoopes, C. G., \& Walterbos, R. A. M. 2000, ApJ, 541, 597

Hora, J. L., Latter, W. B., Allen, L. E., et al. 2004, ApJS, 154, 296

Hunt, L., Bianchi, S., \& Maiolino, R. 2005, A\&A, 434, 849

Israel, F. P., \& Kennicutt, R. C. 1980, Astrophys. Lett., 21, 1

Ivezic, Z., \& Elitzur, M. 1997, MNRAS, 287, 799

Jones, T. J., Woodward, C. E., Boyer, M. L., Gehrz, R. D., \& Polomski, E. 2005, ApJ, 620, 731

Kennicutt, Jr., R. C. 1998, ARA\&A, 36, 189

Kessler, M. F., Steinz, J. A., Anderegg, M. E., et al. 1996, A\&A, 315, L27

Kim, K.-T., \& Koo, B.-C. 2001, ApJ, 549, 979

Kurucz, R. L. 1979, ApJS, 40, 1

Leger, A., \& Puget, J. L. 1984, A\&A, 137, L5

Leitherer, C., Schaerer, D., Goldader, J. D., et al. 1999, ApJS, 123, 3

Li, A., \& Draine, B. T. 2001, ApJ, 554, 778

Magrini, L., Cardwell, A., Corradi, R. L. M., Mampaso, A., \& Perinotto, M. 2001, A\&A, 367, 498

Magrini, L., Corbelli, E., \& Galli, D. 2007a, ArXiv e-prints, 704

Magrini, L., Vilchez, J. M., Mampaso, A., Corradi, R. L. M., \& Leisy, P. 2007b, ArXiv e-prints, 705

Makovoz, D., \& Khan, I. 2005, in Astronomical Data Analysis Software and Systems XIV, ed. P. Shopbell, M. Britton, \& R. Ebert, ASP Conf. Ser., 347, 81

Mathis, J. S., Rumpl, W., \& Nordsieck, K. H. 1977, ApJ, 217, 425

McKee, C. F., \& Williams, J. P. 1997, ApJ, 476, 144

McQuinn, K. B. W., Woodward, C. E., Willner, S. P., et al. 2007, ApJ, 664, 850

Mercer, E. P., Clemens, D. P., Bania, T. M., et al. 2004, ApJS, 154, 328

Neugebauer, G., Habing, H. J., van Duinen, R., et al. 1984, ApJ, 278, L1

Oey, M. S., \& Clarke, C. J. 1998, AJ, 115, 1543

Pahre, M. A., Ashby, M. L. N., Fazio, G. G., \& Willner, S. P. 2004, ApJS, 154, 229

Panagia, N. 1973, AJ, 78, 929

Perez-Gonzalez, P. G., Kennicutt, Jr., R. C., Gordon, K. D., et al. 2006, ArXiv Astrophysics e-prints

Reach, W. T., Rho, J., Jarrett, T. H., \& Lagage, P.-O. 2002, ApJ, 564, 302

Reach, W. T., Rho, J., Tappe, A., et al. 2006, AJ, 131, 1479

Regan, M. W., \& Vogel, S. N. 1994, ApJ, 434, 536

Rice, W., Boulanger, F., Viallefond, F., Soifer, B. T., \& Freedman, W. L. 1990, ApJ, 358, 418

Rieke, G. H., Young, E. T., Engelbracht, C. W., et al. 2004, ApJS, 154, 25

Saken, J. M., Fesen, R. A., \& Shull, J. M. 1992, ApJS, 81, 715

Sloan, G. C., Kraemer, K. E., Price, S. D., \& Shipman, R. F. 2003, ApJS, 147, 379

Tabatabaei, F. S., Beck, R., Krause, M., et al. 2007a, A\&A, 466, 509

Tabatabaei, F. S., Beck, R., Krause, M., et al. 2007b, ArXiv Astrophysics e-prints

Vacca, W. D., Garmany, C. D., \& Shull, J. M. 1996, ApJ, 460, 914

Werner, M. W., Roellig, T. L., Low, F. J., et al. 2004, ApJS, 154, 1

Whitney, B. A., Indebetouw, R., Babler, B. L., et al. 2004, ApJS, 154, 315

Williams, R. M., Chu, Y. ., \& Gruendl, R. 2006, ArXiv Astrophysics e-prints

Willner, S. P., \& Nelson-Patel, K. 2002, ApJ, 568, 679

Wu, H., Cao, C., Hao, C.-N., et al. 2005, ApJ, 632, L79

Yun, M. S., Reddy, N. A., \& Condon, J. J. 2001, ApJ, 554, 803 
S. Verley et al.: Star formation in M33: Spitzer photometry of discrete sources, Online Material p 1

\section{Online Material}


S. Verley et al.: Star formation in M33: Spitzer photometry of discrete sources, Online Material p 2

Table 2. Photometry of known type sources (the flag “-99.0” is used when the photometric centre is farther than 3"' 6 from the nominal position).

\begin{tabular}{|c|c|c|c|c|c|c|c|c|c|c|c|c|c|c|c|}
\hline \multirow[t]{2}{*}{ Type } & \multirow[t]{2}{*}{ Num. } & \multirow{2}{*}{$\begin{array}{c}\text { RA (J2000) } \\
\left({ }^{\circ}\right)\end{array}$} & \multirow{2}{*}{$\begin{array}{c}\operatorname{Dec}(\mathrm{J} 2000) \\
\left({ }^{\circ}\right)\end{array}$} & \multicolumn{2}{|r|}{$3.6 \mu \mathrm{m}$} & \multicolumn{2}{|r|}{$4.5 \mu \mathrm{m}$} & \multicolumn{2}{|r|}{$5.8 \mu \mathrm{m}$} & \multicolumn{2}{|r|}{$8.0 \mu \mathrm{m}$} & & $24 \mu$ & & $\mathrm{H} \alpha$ \\
\hline & & & & $F_{v}(\mathrm{mJy})$ & $\delta r$ & $F_{v}(\mathrm{mJy})$ & $\delta r$ & $F_{v}(\mathrm{mJy})$ & $\delta r$ & $F_{v}(\mathrm{mJy})$ & $\delta r$ & $F_{v}(\mathrm{mJy})$ & $\delta r$ & $F\left(10^{-14} \mathrm{erg} \mathrm{s}^{-1}\right)$ & $\delta r$ \\
\hline ISO & 1 & $01: 33: 59.3$ & $30: 35: 49.2$ & 2.779 & 2.38 & 2.248 & 2.06 & 24.410 & 1.86 & 21.504 & 1.77 & 86.133 & 1.33 & 161.110 & 1.01 \\
\hline ISO & 2 & 01:33:09.8 & $30: 27: 25$ & 4.570 & 1.60 & 3.640 & 1.47 & 13.660 & 1.63 & 46.120 & 0.57 & 106.790 & 1.73 & 59.330 & 0.60 \\
\hline ISO & 3 & $01: 33: 44.5$ & 30:44:38 & 3.350 & 1.14 & 1.507 & 1.39 & 9.098 & 1.16 & 24.896 & 0.95 & 204.660 & 0.41 & 89.796 & 0.86 \\
\hline ISO & 4 & $01: 33: 45.5$ & $30: 36: 51.1$ & 2.000 & 1.97 & 3.220 & 1.51 & 11.200 & 2.11 & 30.649 & 1.93 & 57.035 & 1.94 & 62.180 & 1.39 \\
\hline ISO & 5 & $01: 33: 35.5$ & $30: 39: 30.5$ & 1.598 & 1.30 & 1.566 & 1.20 & 10.291 & 1.53 & 27.896 & 1.43 & 95.377 & 0.88 & 18.056 & 0.76 \\
\hline ISO & 6 & $01: 33: 35.5$ & 30:39:30 & 4.060 & 1.23 & 1.566 & 0.79 & 10.291 & 1.12 & 27.896 & 1.02 & 95.377 & 0.49 & 18.056 & 0.60 \\
\hline ISO & 7 & $01: 33: 28.8$ & $30: 40: 24.7$ & 4.303 & 1.31 & 4.589 & 1.28 & 21.656 & 1.81 & 65.556 & 1.17 & 331.663 & 0.61 & 84.178 & 1.47 \\
\hline ISO & 8 & 01:33:33.9 & $30: 41: 28.3$ & 6.338 & 1.89 & 6.683 & 1.57 & 125.460 & 2.38 & 71.506 & 2.21 & 1434.670 & 0.87 & 394.332 & 2.17 \\
\hline ISO & 9 & 01:33:55.3 & $30: 45: 22$ & 1.226 & 1.72 & 1.184 & 1.68 & 6.228 & 1.57 & 3.322 & 1.91 & 64.093 & 1.16 & .720 & 0.76 \\
\hline ISO & 10 & 01:34:00.2 & 30:40:48.6 & 4.552 & 0.85 & 5.278 & 0.89 & 26.941 & 0.97 & 83 & 0.88 & 493.010 & 0.83 & 9.379 & 0.32 \\
\hline ISO & 14 & 01:34:10.9 & $30: 36: 18.1$ & 4.740 & 1.22 & 0.921 & 1.67 & 5.236 & 1.63 & 13.459 & 1.71 & 48.906 & 0.92 & 2.270 & 2.10 \\
\hline ISO & 16 & 01:33:36.6 & $30: 20: 13.4$ & 3.220 & 0.84 & 2.480 & 0.53 & 10.200 & 0.65 & 1.916 & 0.53 & 79.540 & 0.82 & 5.650 & 0.09 \\
\hline ISO & 19 & 01:33:16.5 & $30: 52: 49.1$ & 4.883 & 0.80 & 10.097 & 0.82 & 27.897 & 0.85 & 70.689 & 0.71 & 942.423 & 0.97 & 2.805 & 0.59 \\
\hline ISO & 21 & 01:33:16.3 & $30: 56: 43.8$ & 0.402 & 2.50 & 88 & 2.48 & 15 & 2.14 & 3.164 & 1.91 & 9.0 & -99.0 & .221 & 2.19 \\
\hline ISO & 23 & 01:34:33.5 & $30: 46: 50.5$ & 14.115 & 2.50 & 12.607 & 1.96 & 25.052 & 0.93 & 183.911 & 1.06 & -99.0 & -99.0 & -99.0 & -99.0 \\
\hline ISO & 24 & 01:34:32.1 & $30: 46: 59.9$ & 9.463 & 0.30 & 5.080 & 0.97 & 55.272 & 0.89 & 167.831 & 0.82 & 264.127 & 1.59 & 313.513 & 0.69 \\
\hline ISO & 25 & 01:34:16.4 & $30: 51: 55.1$ & 3.162 & 1.11 & 3.228 & 1.14 & 35.200 & 1.19 & 19.684 & 1.36 & 224.650 & 0.63 & 98.304 & 0.47 \\
\hline $\mathrm{Hra}$ & 5 & $1: 33: 16.00$ & $+30: 56: 45.9$ & 4.570 & 2.62 & 3.640 & 2.47 & 13.660 & 1.26 & 6.120 & 1.15 & 106.790 & 0.92 & 9.330 & 1.13 \\
\hline $\mathrm{Hra}$ & 7 & $01: 33: 33.62$ & $+30: 32: 08.1$ & 4.938 & 1.04 & 10.096 & 1.06 & 48.730 & 1.17 & 71.289 & 0.99 & 996.074 & 0.43 & 100.845 & 0.84 \\
\hline $\mathrm{Hra}$ & 10 & $01: 33: 36.53$ & $+30: 20: 14.4$ & 1.598 & 0.70 & 1.542 & 0.75 & 9.788 & 0.91 & 27.048 & 0.66 & 94.753 & 0.30 & 18.056 & 0.85 \\
\hline Hra & 11 & $01: 33: 39.18$ & $+30: 38: 06.9$ & 3.220 & 1.50 & 2.480 & 1.21 & 10.200 & 0.84 & 14.362 & 0.85 & 79.540 & 0.64 & 115.650 & 0.74 \\
\hline Hra & 12 & 01:33:42.96 & $+30: 44: 40.6$ & 0.106 & 0.59 & 0.177 & 0.61 & 0.227 & 0.65 & 0.258 & 0.88 & 0.704 & 1.59 & -99.0 & -99.0 \\
\hline $\mathrm{Hra}$ & 13 & 01:33:43.64 & $+30: 39: 07.1$ & 0.074 & 1.79 & 0.262 & 1.77 & 1.519 & 1.68 & 996 & 1.89 & 33.199 & 2.24 & 21.820 & 0.83 \\
\hline $\mathrm{Hra}$ & 14 & 01:33:44.49 & $+30: 44: 38.4$ & 1.424 & 1.50 & 1.130 & 1.10 & 7.748 & 2.09 & 23.673 & 2.44 & 22.730 & 0.56 & 496 & 0.07 \\
\hline $\mathrm{Hra}$ & 16 & :33:47.86 & $+30: 43: 52.6$ & 50 & 1.67 & 1482 & 1.75 & 81 & 1.54 & & 1.30 & 74 & 1.25 & 96 & 0.84 \\
\hline $\mathrm{Hra}$ & 17 & .16 & $+30:$ & & 1.47 & & 1.55 & & 1.96 & & 1.78 & & 0.60 & & 0.38 \\
\hline Hra & 19 & 34 & .0 & 69 & 1.91 & & 2.22 & & 1.73 & & 1.83 & & 1.80 & & 0.29 \\
\hline Hra & 20 & 57 & 18.4 & 19 & 1.63 & 2.2 & 2.32 & 11 & 1.57 & 55 & 1.33 & & 0.61 & & 2.83 \\
\hline $\mathrm{Hra}$ & 22 & .47 & 6.2 & 8. & 1.35 & 94. & 1.20 & 115 & 1.24 & 12 & 1.49 & & 1.11 & & 1.22 \\
\hline $\mathrm{Hra}$ & 27 & .01 & 4 & 79 & 2.78 & & 2.45 & & 2.23 & & 1.96 & & 1.37 & & 1.11 \\
\hline $\mathrm{Hra}$ & 28 & 01:34:02.21 & $+30: 38: 40.7$ & 4.470 & 0.53 & 188 & 0.54 & 26.941 & 0.59 & 78. & 0.26 & 493 & 0.05 & 79 & 0.33 \\
\hline Hra & 29 & 01:34:06.38 & $+30: 41: 45.6$ & 1.266 & 0.70 & 155 & 0.61 & 5 & 0.91 & 50.250 & 1.07 & 23.085 & 0.72 & 758 & 0.70 \\
\hline $\mathrm{Hra}$ & 33 & 01:34:13.73 & $+30: 34: 51.4$ & 1.000 & 1.55 & .924 & 1.28 & 5.719 & 1.33 & 129.290 & 1.42 & 55.358 & 0.29 & 72.270 & 0.47 \\
\hline $\mathrm{Hra}$ & 37 & $01: 34: 33.13$ & $+30: 46: 57.3$ & 7.970 & 0.58 & 0.848 & 0.68 & 5.545 & 0.77 & 15.175 & 0.70 & 101.800 & 0.18 & 122.040 & 1.47 \\
\hline Hra & 40 & 01:34:41.04 & $+30: 43: 29.5$ & 0.364 & 0.96 & 0.442 & 0.88 & 2.860 & 1.08 & 5.227 & 0.98 & 59.380 & 0.80 & 18.960 & 0.35 \\
\hline HII & 1 & $1: 32: 29.5$ & $30: 36: 07.9$ & 0.119 & 1.95 & 181 & 1.92 & 0.330 & 1.27 & 915 & 1.47 & -99.0 & -99.0 & 850 & 0.46 \\
\hline HII & 9 & $2: 45.9$ & $30: 41: 35.5$ & .168 & 1.00 & 080 & 0.92 & 0.130 & 0.58 & 1.009 & 0.52 & .050 & 0.28 & 400 & 0.12 \\
\hline HII & 17 & $3: 10.8$ & 30:18:08.5 & 0.080 & 0.96 & 050 & 0.55 & 0.090 & 0.98 & .700 & 0.78 & -99.0 & -99.0 & .080 & 0.25 \\
\hline HII & 19 & 3:11.2 & $30: 45: 16.4$ & 2.916 & 0.66 & 2.941 & 0.44 & 10.046 & 1.22 & 25.664 & 1.29 & 177.960 & 0.63 & 7.495 & 0.24 \\
\hline HII & 26 & 3:19.0 & $30: 58$ & 199 & 0.68 & .088 & 0.39 & .448 & 0.25 & & 1.06 & & 1.01 & 171 & 0.22 \\
\hline HII & 29 & & & & 1.87 & & 1.82 & & 2.28 & & 2.03 & & 1.84 & & 1.15 \\
\hline HII & 30 & 0 & & & 1.62 & & 1.34 & 0 & 1.72 & 63 & 1.78 & 90 & 1.69 & 92 & 0.24 \\
\hline HII & 37 & & & & 2.52 & & 2.02 & & 1.55 & & 1.77 & & 2.68 & & 1.37 \\
\hline HII & 39 & & & & 0.56 & & 0.63 & & 0.69 & & 0.63 & & 0.64 & & 0.31 \\
\hline HII & 40 & & & & 0.41 & & 0.76 & & 0.27 & & 0.83 & & 0.55 & & 0.18 \\
\hline HII & 47 & & & & 0.36 & & 0.10 & & 0.20 & & 0. & & 1.21 & & 0.30 \\
\hline HII & 49 & :01.7 & 30: & 40 & 2.58 & 120 & 2.31 & 96 & 2.43 & 0 & 1.55 & 34 & 2.02 & 10 & 0.67 \\
\hline HII & 51 & .3 & 31 & 44 & 1.43 & 031 & 1.43 & 6 & 2.3 & 0.442 & 1.82 & 10 & 2.05 & 21 & 0.38 \\
\hline HII & 52 & $34: 06.7$ & $30: 48: 56.4$ & 0.326 & 0.75 & 0.302 & 0.54 & 757 & 1.24 & .450 & 1.48 & .930 & 1.92 & 24.962 & 0.43 \\
\hline HII & 53 & 1:34:09.4 & $30: 34: 17.0$ & 0.820 & 1.61 & 0.597 & 1.66 & 728 & 2.19 & 7.538 & 2.41 & -99.0 & -99.0 & 23.980 & 0.31 \\
\hline HII & 55 & 34:14.4 & $30: 27: 40.3$ & 120 & 1.62 & 41 & 2.39 & 185 & 0.66 & 186 & 2.13 & -99.0 & -99.0 & 0.248 & 0.32 \\
\hline HII & 62 & $4: 36.1$ & $30: 45: 59.6$ & 58 & 1.50 & 110 & 1.20 & 488 & 1.20 & 7 & 1.15 & 609 & 2.38 & 4.619 & 2.12 \\
\hline HII & 64 & 34:37.4 & $30: 34: 54.3$ & 369 & 0.93 & 445 & 0.84 & 35 & 0.84 & 193 & 1.08 & 59.380 & 1.43 & 8.960 & 0.16 \\
\hline HII & 65 & 4:38.5 & $30: 32: 52.3$ & 034 & 0.35 & 027 & 0.12 & 149 & 0.61 & 240 & 0.53 & 0.710 & 1.01 & 1.080 & 0.34 \\
\hline HII & 67 & $4: 39.3$ & $31: 02$ & .051 & 1.93 & 04 & 1.8 & 294 & 1.9 & + & 2.05 & 9.0 & -99.0 & 169 & 0.64 \\
\hline HII & 68 & $1: 34: 39.5$ & $30: 31: 14.2$ & 0.128 & 0.83 & 0.116 & 0.76 & 684 & 0.66 & 1.972 & 0.31 & 21.350 & 1.02 & .090 & 0.33 \\
\hline HII & 69 & $1: 34: 42.2$ & $30: 24: 00.5$ & 0.043 & 0.38 & .046 & 0.29 & 161 & 0.32 & 0.310 & 0.31 & .210 & 0.39 & 620 & 0.28 \\
\hline HII & 70 & $: 42.5$ & 30: & 110 & 0.59 & 40 & 0.89 & & 0.1 & 19 & 0.72 & 99.0 & -99.0 & 999 & 0.12 \\
\hline HII & 71 & 4:45.5 & 24.4 & 0.200 & 0.41 & 159 & 0.18 & & 0.24 & & 0.21 & & 0.75 & .046 & 0.49 \\
\hline HII & 72 & :49.2 & 30 & J2 & 0.85 & & 0.81 & & 0.7 & & 1.08 & & 1.40 & 430 & 0.63 \\
\hline HII & 75 & 1.6 & $30: 40: 02.3$ & 069 & 0.3 & .053 & 0.35 & & 0.64 & 0.940 & 0.42 & 99.0 & -99.0 & 1.456 & 0.49 \\
\hline HII & 76 & 1:35:02.4 & 31:00:43.4 & 0.067 & 1.17 & 0.044 & 1.00 & 0.153 & 1.03 & 0.141 & 1.16 & -99.0 & -99.0 & 2.687 & 0.70 \\
\hline SN & 25 & 3:23.8 & 30 & & 1.40 & 0.380 & 0.96 & & 0.81 & 1.325 & 1.87 & 1.947 & 1.55 & 3.310 & 0.95 \\
\hline SNo & 43 & 3:41.7 & & & 2.56 & & .21 & & 2.09 & 18.670 & 0.61 & 11.060 & 0.54 & 14.907 & 1.82 \\
\hline SNo & 53 & $1: 33: 54.5$ & $30: 33: 47.7$ & 0.134 & 2.67 & 0.070 & 2.84 & 0.300 & 2.71 & 0.733 & 2.85 & -99.0 & -99.0 & -99.0 & -99.0 \\
\hline
\end{tabular}


S. Verley et al.: Star formation in M33: Spitzer photometry of discrete sources, Online Material p 3

Table 2. continued.

\begin{tabular}{|c|c|c|c|c|c|c|c|c|c|c|c|c|c|c|c|}
\hline \multirow[t]{2}{*}{$\overline{\text { Type }}$} & \multirow[t]{2}{*}{ Num. } & \multirow{2}{*}{$\begin{array}{c}\text { RA (J2000) } \\
\left({ }^{\circ}\right) \\
\end{array}$} & \multirow{2}{*}{$\begin{array}{c}\text { Dec (J2000) } \\
\left({ }^{\circ}\right) \\
\end{array}$} & \multicolumn{2}{|r|}{ 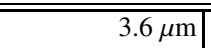 } & \multicolumn{2}{|r|}{ 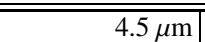 } & \multicolumn{2}{|c|}{$\overline{5.8 \mu \mathrm{m}}$} & \multicolumn{2}{|r|}{ 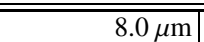 } & \multicolumn{2}{|r|}{$\overline{24 \mu \mathrm{m}}$} & \multicolumn{2}{|r|}{$\overline{\overline{\mathrm{H} \alpha}}$} \\
\hline & & & & $F_{v}(\mathrm{mJy})$ & $\delta r$ & $F_{v}(\mathrm{mJy})$ & $\delta r$ & $F_{v}(\mathrm{mJy})$ & $\delta r$ & $F_{v}(\mathrm{mJy})$ & $\delta r$ & $F_{v}(\mathrm{mJy})$ & $\delta r$ & $F\left(10^{-14} \mathrm{erg} \mathrm{s}^{-1}\right)$ & $\delta r$ \\
\hline SNR & 13 & $1: 33: 23.78$ & $30: 26: 11.60$ & 0.550 & 1.42 & 0.380 & 0.99 & 0.890 & 1.00 & 1.319 & 1.92 & 1.947 & $\begin{array}{ll}1.68 \\
\end{array}$ & 3.310 & 0.97 \\
\hline $\mathrm{PNe}$ & 4 & $01: 32: 39.80$ & $+30: 37: 41.0$ & 0.017 & 0.23 & 18 & 0.79 & 23 & 1.16 & 66 & 0.69 & .000 & 6.74 & .164 & 0.18 \\
\hline $\mathrm{PNe}$ & 60 & $01: 33: 44.38$ & $+30: 20: 23.7$ & 50 & 2.64 & 35 & 2.41 & 58 & 2.73 & 39 & 2.45 & 361 & 0.81 & 0.220 & 0.19 \\
\hline $\mathrm{PNe}$ & 79 & $01: 33: 54.93$ & $+30: 47: 13.4$ & 0.156 & 1.61 & 84 & 1.46 & 07 & 1.86 & 99 & 1.01 & 000 & 7.18 & -99.0 & -99.0 \\
\hline $\mathrm{PNe}$ & 85 & 01:33:58.38 & $+30: 55: 05.2$ & 0.050 & 2.34 & 41 & 1.71 & 17 & 1.61 & 0.698 & 1.75 & 389 & 1.29 & 676 & 0.87 \\
\hline $\mathrm{PNe}$ & 106 & 01:34:12.15 & $+30: 28: 57.1$ & 0.160 & 2.65 & 90 & 2.85 & & 2.56 & 74 & 2.90 & 79 & 3.43 & 181 & 1.37 \\
\hline $\mathrm{PNe}$ & 112 & 01:34:15.15 & $+30: 44: 57.4$ & 0.189 & 2.04 & & 2.10 & & 2.15 & & 2.34 & 0 & 7.62 & 35 & 0.06 \\
\hline $\mathrm{PNe}$ & 128 & 01:34:25.55 & $+30: 40: 10.9$ & 0.248 & 0.71 & 57 & 0.65 & & 0.39 & 77 & 0.28 & 0.394 & 0.42 & 275 & 0.37 \\
\hline $\mathrm{PNe}$ & 133 & 01:34:31.49 & $+31: 05: 24.0$ & 23 & 0.54 & 12 & 0.69 & $0.06 /$ & 0.48 & 0.1 & 0.07 & 0.000 & 6.63 & 0.148 & 0.17 \\
\hline $\mathrm{PNe}$ & 139 & 01:34:37.89 & $+30: 27: 00.7$ & 0.140 & 2.20 & 0.060 & 2.20 & 0.114 & 1.73 & 0.160 & 0.88 & 0.113 & 0.60 & 0.210 & 0.20 \\
\hline
\end{tabular}


Table 3. Photometry of $24 \mu \mathrm{m}$ sources (the flag “- -99.0 " is used when the photometric centre is farther than 3"' 6 from the nominal position).

\begin{tabular}{|c|c|c|c|c|c|c|c|c|c|c|c|c|c|c|}
\hline \multirow[t]{2}{*}{ Num. } & \multirow{2}{*}{$\begin{array}{c}\text { RA (J2000) } \\
\left({ }^{\circ}\right)\end{array}$} & \multirow{2}{*}{$\begin{array}{c}\operatorname{Dec}(\mathrm{J} 2000) \\
\left(^{\circ}\right)\end{array}$} & \multicolumn{2}{|r|}{$3.6 \mu \mathrm{m}$} & \multicolumn{2}{|r|}{$4.5 \mu \mathrm{m}$} & \multicolumn{2}{|r|}{$5.8 \mu \mathrm{m}$} & \multicolumn{2}{|r|}{$8.0 \mu \mathrm{m}$} & \multicolumn{2}{|r|}{$24 \mu \mathrm{m}$} & \multicolumn{2}{|r|}{$\mathrm{H} \alpha$} \\
\hline & & & $F_{v}(\mathrm{mJy})$ & $\delta r$ & $F_{v}(\mathrm{mJy})$ & $\delta r$ & $F_{v}(\mathrm{mJy})$ & $\delta r$ & $F_{v}(\mathrm{mJy})$ & $\delta r$ & $F_{v}(\mathrm{mJy})$ & or & $F\left(10^{-14} \mathrm{erg} \mathrm{s}^{-1}\right)$ & $\delta r$ \\
\hline 1 & 23.3749767 & 30.3099105 & 0.2 & 2.846 & 0.13 & 2.799 & 0.76 & 2.934 & 2.71 & 2.762 & 23.59 & 0.022 & -99.0 & -99.0 \\
\hline 2 & 23.3614272 & 30.2907556 & 0.06 & 0.646 & 0.069 & 0.54 & 33 & 0.824 & 1.46 & 0.956 & 0.75 & 0.0 & 0.32 & 0.2 \\
\hline 3 & 23.3721103 & 30.3016896 & 0.21 & 1.196 & 0.1 & 1.001 & 0.58 & 0.522 & 2.27 & 0.796 & 4.59 & 0.022 & 5.583 & 0.24 \\
\hline 4 & 23.3329204 & 30.3053012 & -99.0 & -99.0 & -99.0 & -99.0 & 0.364 & 0.527 & 0.963 & 1.04 & 1.286 & 0.122 & -99.0 & -99.0 \\
\hline 5 & 23.3358182 & 30.3040334 & 0.021 & 0.72 & 0.052 & 0.73 & 0.105 & 0.652 & -99.0 & -99.0 & 0.7 & 0.073 & -99.0 & -99.0 \\
\hline 6 & 23.4435711 & 30.3038256 & 0.046 & 0.592 & 0.04 & 0.586 & 0.148 & 0.62 & 0.14 & 0.643 & 0.29 & 0.002 & -99.0 & -99.0 \\
\hline 7 & 23.4487444 & 30.3048739 & -99.0 & -99.0 & -99.0 & -99.0 & -99.0 & -99.0 & -99.0 & -99.0 & 0.67 & 0.077 & -99.0 & -99.0 \\
\hline 8 & 23.4024463 & 30.3367871 & 3.22 & 0.433 & 2.48 & 0.557 & 4.57 & 0.989 & 11.998 & 0.954 & 79.54 & 0.001 & 53.569 & 0.424 \\
\hline 9 & 23.3768792 & 3196512 & 0.051 & 1.954 & 0.042 & 2.02 & 21 & 1.599 & 2.33 & 0.926 & 0.82 & 0.204 & 1.16 & 2.558 \\
\hline 10 & 23.3843288 & 30.3366678 & 0.205 & 2.028 & 0.191 & 1.907 & 1.951 & 1.054 & 5.84 & 0.756 & 4.258 & 0.16 & 2.59 & 1.505 \\
\hline 11 & 23.48185 & .330103 & 6 & 0.647 & 14 & 0.489 & 0.62 & 0.823 & 2.13 & 1.002 & 64 & 0.031 & 1.01 & 0.243 \\
\hline 12 & 23.4718432 & 314455 & 0.074 & 1.463 & 553 & 31 & 11 & 0.56 & .47 & 0.774 & 24 & 0.016 & 99.0 & -99.0 \\
\hline 13 & 3860886 & 334663 & -99.0 & -99.0 & 027 & 0.932 & 26 & 1.079 & 2.06 & 1.164 & 44 & 0.121 & 1.6 & 1.766 \\
\hline 14 & 3.4064918 & 470677 & 0.65 & 2.978 & 22 & 2.634 & 2. & 0.68 & 14.48 & 0.386 & 6.9 & 0.229 & 2.49 & 0.929 \\
\hline 15 & 23.3513249 & .3401814 & 0.23 & 2.685 & 16 & 1.825 & 0.137 & 0.609 & 0.14 & 0.456 & 1.05 & 0.001 & 99.0 & -99.0 \\
\hline 16 & 23.3882322 & .3414916 & -99.0 & -99.0 & -99.0 & -99.0 & 0.28 & 0.32 & 2.0 & 0.519 & 0.9 & 0.002 & 4.189 & 0.71 \\
\hline 17 & 23.4370169 & 30.3513656 & 1.15 & 1.087 & 0.86 & 0.966 & 1.4 & 0.939 & 1.374 & 1.071 & 2.04 & 0.003 & 2.951 & 2.055 \\
\hline 18 & 23.4379955 & 30.3604276 & 0.48 & 1.602 & 0.4 & 1.196 & 1.39 & 1.172 & 4.22 & 1.186 & 17.56 & 0.009 & 22.336 & 0.215 \\
\hline 19 & 23.410961 & 30.3512346 & 0.669 & 0.362 & 0.765 & 0.311 & 1.17 & 0.594 & 1.4 & 0.622 & 3.05 & 0.043 & -99.0 & -99.0 \\
\hline 20 & 23.2919766 & 30.3854636 & -99.0 & -99.0 & 0.7 & 2.58 & 4.79 & 0.849 & 15.14 & 2.412 & 25.14 & 0.329 & 21.901 & 0.777 \\
\hline 21 & 23.3234794 & 30.3571074 & 0.65 & 1.11 & 0.49 & 0.847 & 0.19 & 0.281 & 19 & 0.639 & 1.02 & 0.11 & 0.056 & 0.51 \\
\hline 22 & 23.414503 & 30.3611675 & 0.22 & 0.289 & 24 & 0.483 & 0 & 0.562 & 0 & 0.613 & 07 & 0.002 & 99.0 & -99.0 \\
\hline 23 & 23.4145537 & 7926 & & 1.408 & 42 & 1.42 & 48 & 413 & 3 & 1.234 & & 0.041 & & 1.477 \\
\hline 24 & 23.48 & & .0 & -99.0 & & -99.0 & & 2. & 11. & 2.034 & & 0.055 & & 0.242 \\
\hline 25 & 23.43 & 494 & -99.0 & -99.0 & -99.0 & -99.0 & t & 0.814 & 0. & 0.854 & 1.49 & 0.002 & -99.0 & -99.0 \\
\hline 26 & 383 & 194 & 0 & 2.189 & 0 & 2.383 & & 1. & 1 & 2.584 & & 0.029 & & 2.557 \\
\hline 27 & 787 & 33 & & 2.343 & & 0.428 & & 0.339 & 0.2 & 0.381 & & 0.001 & 99.0 & -99.0 \\
\hline 28 & 23.2961441 & 633 & -99.0 & -99.0 & -99.0 & -99.0 & & 2 & & 2.201 & 1.11 & 0.097 & 99.0 & -99.0 \\
\hline 29 & 23.2797677 & 63205 & -99.0 & -99.0 & -99.0 & -99.0 & -99.0 & -99.0 & 7.9 & 1.122 & 6.7 & 0.037 & 1.62 & 2.011 \\
\hline 30 & 23.4006862 & 30.3774629 & -99.0 & -99.0 & -99.0 & -99.0 & 0.16 & 0.3 & 0.69 & 0.453 & 2.0 & 0.001 & .472 & 1.303 \\
\hline 31 & 23.5000231 & 30.37729 & 0.13 & 0.697 & 0.12 & 0.685 & 0.4 & 1.315 & 0.96 & 1.426 & 0.38 & 0.001 & -99.0 & -99.0 \\
\hline 32 & 23.2887017 & 30.3916345 & 0.56 & 0.913 & 42 & 1.35 & 1.203 & 0.875 & 3.357 & 0.7 & 19.81 & 1.467 & 17.273 & 2.536 \\
\hline 33 & 23.3178801 & 3847881 & 3.3 & 0.921 & 22 & 0.893 & 1.8 & 0.948 & 1.84 & 0.824 & 2.7 & 0.001 & 0. & 0.899 \\
\hline 34 & 23.3616848 & 3870248 & 80 & 0.621 & 34 & 575 & & 03 & 2.1 & 0.638 & 2.0 & 0.032 & -99.0 & -99.0 \\
\hline 35 & 23.4989082 & 3848985 & 0.116 & 0.891 & 069 & 0.948 & 0.197 & 18 & 61 & 1.738 & 0. & 0.002 & 26 & 2.811 \\
\hline 36 & 7 & & & 99.0 & & -99.0 & & & & & & 0.077 & & -99.0 \\
\hline 37 & 2 & & & -9 & & -99.0 & & & & -9 & 97 & 0.006 & & -99.0 \\
\hline 38 & 23.5 & & -99.0 & -99.0 & 0 & -99.0 & -9 & -99.0 & -99.0 & -99.0 & & 0.001 & -99.0 & -99.0 \\
\hline 39 & 4 & & & & 0.07 & 0.535 & & & & 0.709 & & 0.025 & & 0.32 \\
\hline 40 & 6 & & & & & & & & & 1. & & 2.577 & & 0.5 \\
\hline 41 & 4 & & & & & 1. & & & & 1. & & 0.127 & 29 & 1.511 \\
\hline 42 & 48 & 54 & -99.0 & -99.0 & -99.0 & -99.0 & -99 & -99.0 & 1.003 & 0.653 & 0.53 & 0.117 & & 2.508 \\
\hline 43 & 23.54 & 97 & & 0.798 & 0 & 0.457 & & 0 & 4.7 & 0.831 & 4.7 & 0.001 & .597 & 0.325 \\
\hline 44 & 27 & 49 & 0.038 & .739 & 27 & 0.725 & & 0.432 & & 0.526 & 1.15 & 0.055 & .457 & 2.145 \\
\hline 45 & 23.5583747 & .4298619 & & 0.861 & 0.38 & 0.859 & 1.39 & 1.087 & 1.495 & 1.186 & 3.05 & 0.033 & 3.4 & 2.687 \\
\hline 46 & 23.397946 & 781 & & 0.654 & 0 & 0.479 & & 0.728 & 5.6 & 0.622 & 7.55 & 0.014 & 7.2 & 0.314 \\
\hline 47 & 23.5581556 & .5800716 & -99.0 & -99.0 & -99.0 & -99.0 & -99.0 & -99.0 & -99.0 & -99.0 & 199.76 & 0.08 & -99.0 & -99.0 \\
\hline 48 & 23.3472539 & 4430213 & -99.0 & -99.0 & -99.0 & -99.0 & 0.6 & 1.157 & 1.196 & 1.249 & 0.851 & 0.152 & 2.59 & 2.223 \\
\hline 49 & 23.2869175 & 4975933 & -99.0 & -99.0 & -99.0 & -99.0 & 21.73 & 1.073 & 124.86 & 2.16 & 5.65 & 0.065 & 6.24 & 0.475 \\
\hline 50 & 497 & 09 & & 2.8 & & 545 & & 66 & & 1.384 & 14 & 0.0 & -99.0 & -99.0 \\
\hline 51 & & & -99.0 & -9 & 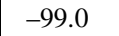 & -99.0 & & -99.0 & -99.0 & -99.0 & 25 & 0.009 & & 2.399 \\
\hline 52 & 23.48 & & -99.0 & -99.0 & -99.0 & -99.0 & -99.0 & -99.0 & -99.0 & -99.0 & 1. & 0.229 & -99.0 & -99.0 \\
\hline 53 & & & & 0.7 & & 0.143 & 0.9 & 0.377 & & 0.477 & & 0.001 & & 0.371 \\
\hline 54 & & & 0.093 & & & 0.836 & & & & 0.684 & 3.0 & 0.978 & & 1.912 \\
\hline 55 & 23.48 & & 2.628 & 0.6 & 2.788 & 0.668 & 2.771 & & 2.649 & 0.713 & 1.4 & 0.127 & -99.0 & -99.0 \\
\hline 56 & & & 1.6 & & & & & & 4. & 0.667 & 11.23 & 0.004 & 0.446 & 2.689 \\
\hline 5 & 23.54 & 30 & & & -99.0 & -99.0 & & & 4.4 & 0.639 & 0.87 & 0.088 & 1.46 & 0.398 \\
\hline 58 & & & & & & 1.723 & & & 6.8 & 0.962 & 44 & 0.048 & -99.0 & -99.0 \\
\hline 59 & 23.4668067 & 30.45 & & 1. & & 1.019 & & 1. & 0. & 0.758 & 0.36 & 0.001 & -99.0 & -99.0 \\
\hline 60 & 23.3902028 & .5356536 & -99.0 & -99.0 & -99 & -99.0 & 71.2 & 1.952 & 395 & 0.32 & 250.56 & 0.411 & 136.42 & 1.246 \\
\hline 61 & 23.5639999 & 30.4711516 & 2.95 & 0.323 & & 0.284 & 46 & 0.609 & 4.247 & 0.323 & 17.56 & 0.0 & 1.805 & 0.192 \\
\hline 62 & 23.5964784 & 30.4621603 & -99.0 & -99.0 & -99.0 & -99.0 & 0.031 & 0.722 & 0.358 & 1.745 & 0.29 & 0.001 & -99.0 & -99.0 \\
\hline 63 & 23.6059902 & 30.4649241 & -99.0 & -99.0 & -99.0 & -99.0 & -99.0 & -99.0 & 0.155 & 1.481 & 0.3 & 0.129 & -99.0 & -99.0 \\
\hline 64 & 23.295885 & 30.4991531 & -99.0 & -99.0 & -99.0 & -99.0 & 5.68 & 0.751 & 15.95 & 0.573 & 23.08 & 0.162 & -99.0 & -99.0 \\
\hline
\end{tabular}


Table 3. continued.

\begin{tabular}{|c|c|c|c|c|c|c|c|c|c|c|c|c|c|c|}
\hline \multirow[t]{2}{*}{ Num. } & \multirow{2}{*}{$\begin{array}{c}\text { RA (J2000) } \\
\left({ }^{\circ}\right)\end{array}$} & \multirow{2}{*}{$\begin{array}{c}\operatorname{Dec}(\mathrm{J} 2000) \\
\left({ }^{\circ}\right)\end{array}$} & \multicolumn{2}{|r|}{$3.6 \mu \mathrm{m}$} & \multicolumn{2}{|r|}{$4.5 \mu \mathrm{m}$} & \multicolumn{2}{|r|}{$\overline{5.8 \mu \mathrm{m}}$} & \multicolumn{2}{|r|}{$8.0 \mu \mathrm{m}$} & \multicolumn{2}{|r|}{$24 \mu \mathrm{m}$} & & $\overline{\mathrm{H} \alpha}$ \\
\hline & & & $F_{V}(\mathrm{mJy})$ & $\delta r$ & $F_{v}(\mathrm{mJy})$ & $\delta r$ & $F_{v}(\mathrm{mJy})$ & or & $F_{v}(\mathrm{mJy})$ & or & $F_{v}(\mathrm{mJy})$ & $\delta r$ & $F\left(10^{-14} \mathrm{erg} \mathrm{s}^{-1}\right)$ & $\delta r$ \\
\hline 65 & 23.4373183 & 30.6031596 & -99.0 & \begin{tabular}{|c|}
-99.0 \\
\end{tabular} & -99.0 & -99.0 & 75.28 & 0.166 & -99.0 & -99.0 & 163.3 & 0.05 & -99.0 & -99.0 \\
\hline 66 & 23.6070908 & 30.4717673 & & 0.558 & & 0.564 & 92 & 0.795 & & 0.728 & 18 & 0.001 & -99.0 & -99.0 \\
\hline 67 & 23.5731509 & 30.4732955 & & 0.953 & & 0.584 & 59 & 0.68 & & 0.318 & 11 & 0.001 & -99.0 & -99.0 \\
\hline 68 & 23.2896084 & $.48 \%$ & 29 & 1.955 & & 1.435 & 36 & 0.659 & 911 & 0.672 & 3.099 & 0.097 & 0.4 & 0.392 \\
\hline 69 & 23.3472862 & 30.5266611 & 4 & 0.692 & 51 & 0.801 & 48 & 0.346 & 445 & 0.784 & 14.31 & 0.407 & 12.63 & 0.447 \\
\hline 70 & 23.3742461 & 30.530412 & .63 & 0.799 & 55 & 2.92 & 98.58 & 0.532 & -99.0 & -99.0 & 300.19 & 1.624 & 146.03 & 1.68 \\
\hline 71 & 23.4139809 & 30.49824 & 27 & 2.12 & 0.09 & 1.431 & 1.32 & 1.484 & 10.17 & 1.014 & 3.82 & 0.186 & -99.0 & -99.0 \\
\hline 72 & 23.4523366 & 30.6610505 & 175.3 & 1.792 & 102.97 & 2.683 & 148.28 & 1.079 & -99.0 & -99.0 & 281.52 & 0.302 & -99.0 & -99.0 \\
\hline 73 & 23.266931 & 30.4825374 & 0.16 & 2.151 & -99.0 & -99.0 & -99.0 & -99.0 & 0.21 & 0.265 & 1.1 & 0.001 & 3.16 & 0.933 \\
\hline 74 & 23.301739 & 30.5073557 & -99.0 & -99.0 & -99.0 & -99.0 & -99.0 & -99.0 & -99.0 & -99.0 & 31.08 & 1.263 & -99.0 & -99.0 \\
\hline 75 & 23.4985862 & 30.5639235 & -99.0 & -99.0 & -99.0 & -99.0 & -99.0 & -99.0 & -99.0 & -99.0 & 40.67 & 0.82 & 104.46 & 2.004 \\
\hline 76 & 23.4844389 & 30.4914555 & 1.14 & 1.222 & 0.73 & 1.264 & 1.04 & 1.36 & 7.74 & 1.289 & 5.23 & 0.017 & -99.0 & -99.0 \\
\hline 77 & 23.39711 & 30.4975299 & 4 & 1.51 & 0.3 & 1.638 & 52 & 1.431 & 3.088 & 1.283 & 11.54 & 0.01 & 4.919 & 1.132 \\
\hline 78 & 23.2681679 & 30.49 & -99.0 & -99.0 & -6 & -99.0 & 0.36 & 1.76 & & 1.377 & 35 & 0.135 & 3.22 & 2.78 \\
\hline 79 & 23.4 & 30.5 & 0 & -99.0 & -6 & -99.0 & 14.97 & 0.719 & 9 & 0.405 & 28 & 0.367 & .484 & 2.145 \\
\hline 80 & 23.2 & 30.5 & & -99.0 & & 2.157 & & 2.538 & & 2.702 & 11. & 0.573 & 469 & 0.349 \\
\hline 81 & 23.2 & 5 & & 0.5 & 0. & 0.829 & 2.95 & 0.65 & & 1.023 & & 0.58 & 7.385 & 0.281 \\
\hline 82 & 23 & 30.4 & & 05 & 1 & 1.165 & 1.5 & 1.155 & & 0.942 & 1.42 & 0.098 & 5.62 & 1.089 \\
\hline 83 & 23.40 & 0.5 & 0.095 & 1.45 & 0. & 1.468 & 0.95 & 2.886 & 3.161 & 2.91 & 0.11 & 2.76 & 2.763 & 2.52 \\
\hline 84 & 23.4 & 93 & 121.02 & 2.091 & 94.48 & 1.707 & -99.0 & -99.0 & -99.0 & -99.0 & 333.39 & 2.151 & 99.0 & -99.0 \\
\hline 85 & 23.5536876 & 742 & 26 & 0.149 & 2.4 & 0.309 & 3.11 & 0.516 & 3.56 & 0.454 & 3.07 & 0.0 & 157 & 2.899 \\
\hline 86 & 23.2613307 & 3575 & -99.0 & -99.0 & -99.0 & -99.0 & 1.489 & 2.836 & 0.801 & 1.873 & .05 & 0.002 & .673 & 0.509 \\
\hline 87 & 23.3278802 & .4928602 & 0.22 & 0.409 & 0.19 & 0.431 & 0.17 & 0.489 & 3.897 & 1.014 & 93 & 0.003 & 99.0 & -99.0 \\
\hline 88 & 23.4088097 & 30.5005227 & -99.0 & -99.0 & 0.068 & 1.46 & 0.46 & 0.79 & 1.182 & 0.669 & .98 & 0.077 & -99.0 & -99.0 \\
\hline 89 & 23.5064687 & 30.51 & 0.38 & 2.851 & 0.13 & 2.681 & 2.5 & 1.2 & 12.1 & 1.156 & 4.96 & 0.001 & -99.0 & -99.0 \\
\hline 90 & 23.41 & 30.50 & -99.0 & -99.0 & -99.0 & -99.0 & 2.54 & 0.344 & 51 & 0.348 & 4.139 & 0.094 & -99.0 & -99.0 \\
\hline 91 & 23.4 & 30.5 & 252 & 0.653 & 0.219 & 0.585 & 29.66 & 0.305 & 7 & 1.577 & 61.36 & 0.496 & 65.3 & 1.3 \\
\hline 92 & 23.31 & 30.5 & 9.0 & -99.0 & 0.05 & 0.34 & 0.167 & 0.299 & 0.239 & 0.253 & & 0.005 & -99.0 & -99.0 \\
\hline 93 & & 30.5 & & 0.747 & & 0.305 & 486 & 0.919 & & 1.419 & 92 & 0.043 & 53.9 & 2.256 \\
\hline 94 & 23.2 & 8 & -1 & -99.0 & -99.0 & -99.0 & 0.276 & 1.945 & & 1.559 & 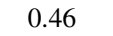 & 0.001 & 5.129 & 1.937 \\
\hline 95 & & & & 0.348 & & 0.381 & & 0.298 & & 0.462 & 0.4 & 0.001 & -99.0 & -99.0 \\
\hline 96 & 23.4 & & & 2.3 & & 1.503 & & 1.485 & & 1.4 & 11 & 1.584 & 99.0 & -99.0 \\
\hline 97 & 2 & & & 2 & 1.3 & 2.681 & 59.46 & 2.646 & 3. & 2.869 & 133.93 & 0.636 & 1 & 0.617 \\
\hline 98 & 23.35 & 82 & 23 & 2.49 & 0. & 1.815 & 0.67 & 2.743 & 2.544 & 2.807 & 3.18 & 0.0 & 13.02 & 2.318 \\
\hline 99 & 23.66 & 99 & 0 & 1.869 & 0.16 & 1.439 & 59 & 1.599 & 9.37 & 1.683 & 21.35 & 0.011 & 1.09 & 0.833 \\
\hline 100 & 158 & 99 & & 2.317 & 1.65 & 2.619 & 8.89 & 1.388 & 40.76 & 2.247 & 14.65 & 0.014 & .438 & 1.65 \\
\hline 101 & 23.5920186 & .5218368 & -99.0 & -99.0 & -99.0 & -99.0 & 1.79 & 1.288 & 6.79 & 1.17 & 8.89 & 0.001 & -99.0 & -99.0 \\
\hline 102 & 23.4374512 & 62 & 0.09 & 1.256 & 0.055 & 1.134 & 1. & 1.304 & & 0.749 & 67 & 0.002 & -99.0 & -99.0 \\
\hline 103 & 23.2701529 & 071 & -99.0 & -99.0 & -99.0 & -99.0 & 0.82 & 0.248 & 4.756 & 0.433 & 0.98 & 0.001 & 0.741 & 1.822 \\
\hline 104 & 23.5721108 & 107 & 7.97 & 0.588 & & 0.7 & 41.31 & 1.269 & 129.61 & 0.502 & 101.8 & 0.592 & 22.04 & 1.412 \\
\hline 105 & 327 & 48 & & 2.923 & -99.0 & -99.0 & 59 & 0.764 & 0.819 & 0.474 & 10 & 0.001 & .303 & 2.72 \\
\hline 106 & 52 & 3 & & & & 0.49 & & 681 & & 2 & & 001 & 0 & 99.0 \\
\hline 107 & & & & & & 0.606 & & 4 & & & & 59 & 1 & 1.092 \\
\hline 108 & 4 & & & & & 15 & & 0.268 & & 4 & 0. & .002 & 0 & -99.0 \\
\hline 109 & 5 & & & & & 0.671 & & 1.491 & 1 & 2 & 5 & .014 & & 0.604 \\
\hline 110 & 2 & 9 & & -9 & -6 & -99.0 & 1. & 2.648 & & 4 & 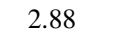 & .003 & 99.0 & -99.0 \\
\hline 111 & 8 & & 0 & -99.0 & -9 & -99.0 & & 1.368 & 0 & 3 & 1.1 & 0.003 & 0 & -99.0 \\
\hline 112 & 665 & 8 & -9 & -99.0 & -99.0 & -99.0 & 2 & 0.397 & 3 & 0.566 & 0.718 & 0.021 & 2.797 & 2.522 \\
\hline 113 & 1628 & 3 & & 0.655 & 1 & 1.562 & 4.189 & 1.038 & 18.641 & 0.687 & 58.94 & 0.284 & -99.0 & -99.0 \\
\hline 114 & .4990498 & 3 & & 2.417 & 0 & 2.362 & -99.0 & -99.0 & 28.15 & 2.314 & 24.02 & 1.083 & 40.02 & 0.359 \\
\hline 115 & 23.5569419 & .5620427 & & 2.242 & 31 & 2.27 & 8.269 & 1.338 & 169.48 & 2.44 & 233.02 & 0.596 & 65.698 & 0.847 \\
\hline 116 & 23.4184798 & .5264105 & -99.0 & -99.0 & -99.0 & -99.0 & 1.19 & 1.196 & 1.853 & 0.427 & 1.51 & 0.062 & -99.0 & -99.0 \\
\hline 117 & .4358347 & .522334 & 76 & 0.9 & 0.1 & 1.434 & 0.726 & 0.214 & 7 & 0.365 & 0.842 & 0.125 & -99.0 & -99.0 \\
\hline 118 & 23.5937772 & 30.5448368 & -99.0 & -99.0 & -99.0 & -99.0 & -99.0 & -99.0 & -99.0 & -99.0 & 15.66 & 0.015 & 3.548 & 2.293 \\
\hline 119 & .5093193 & 52338 & & 2.403 & 047 & 2.069 & 0 & 1.348 & & 0.426 & 5 & 0.088 & 6.01 & 1.091 \\
\hline 120 & 23.25 & 75 & & 1.006 & 0.00 & 1.07 & 0.56 & 1.086 & 1.93 & 1.389 & 4.33 & 0.022 & 3.35 & 2.21 \\
\hline 121 & 23.31 & 26 & 0.52 & 2.261 & -99.0 & -99.0 & 1.59 & 1.832 & 3.432 & 1.224 & 12.36 & 0.037 & -99.0 & -99.0 \\
\hline 122 & 23.50 & 29 & -99.0 & -99.0 & -99.0 & -99.0 & 10.42 & 1.884 & 96 & 1.662 & 26.69 & 0.191 & .229 & 0.393 \\
\hline 123 & 34 & & & 1.363 & -99.0 & -99.0 & -99.0 & -99.0 & -99.0 & -99.0 & 2.4 & 0.003 & -99.0 & -99.0 \\
\hline 124 & & 9 & 0.523 & 2.306 & 0.3 & 2.593 & & 1.034 & & 0. & 2.0 & 0.014 & & 0.741 \\
\hline 125 & 96 & 66 & & 0.612 & & 0.5 & & 1.135 & 3.942 & 0.646 & 2.99 & 0.032 & 6.592 & 0.363 \\
\hline 126 & .2724933 & 6 & & 0.442 & 1.516 & 0.443 & & 0.386 & & & 0.38 & 0.001 & & -99.0 \\
\hline 127 & .4488423 & 0.5 & & & & 1.408 & 7.312 & 0.333 & 17.945 & 0.126 & 81.43 & 0.001 & 5.135 & 0.844 \\
\hline 128 & .2576171 & 59 & 0.3 & 2.146 & & 0.985 & & 0.685 & 4.473 & 0.338 & 5.36 & 0.134 & -99.0 & -99.0 \\
\hline 12 & & & & & & 2.333 & 27.5 & 1.469 & -99.0 & & & 2.746 & 1.834 & 0.589 \\
\hline 130 & 23.6751983 & 30.5403655 & 0.1 & 0.355 & -99.0 & -99.0 & 0.45 & 0.443 & 2.91 & 0.375 & 1.155 & 0.049 & 2.22 & 2.378 \\
\hline
\end{tabular}


Table 3. continued.

\begin{tabular}{|c|c|c|c|c|c|c|c|c|c|c|c|c|c|c|}
\hline \multirow[t]{2}{*}{$\overline{\text { Num. }}$} & \multirow{2}{*}{$\begin{array}{c}\text { RA (J2000) } \\
\left({ }^{\circ}\right) \\
\end{array}$} & \multirow{2}{*}{$\begin{array}{c}\text { Dec (J2000) } \\
\left(^{\circ}\right) \\
\end{array}$} & \multicolumn{2}{|r|}{$3.6 \mu \mathrm{m}$} & \multicolumn{2}{|r|}{$4.5 \mu \mathrm{m}$} & \multicolumn{2}{|r|}{$\overline{5.8 \mu \mathrm{m}}$} & \multicolumn{2}{|r|}{$8.0 \mu \mathrm{m}$} & \multicolumn{2}{|r|}{$24 \mu \mathrm{m}$} & & $\overline{\mathrm{H} \alpha}$ \\
\hline & & & $F_{v}(\mathrm{mJy})$ & $\delta r$ & $F_{v}(\mathrm{mJy})$ & $\delta r$ & $F_{v}(\mathrm{mJy})$ & $\delta r$ & $F_{v}(\mathrm{mJy})$ & $\delta r$ & $F_{v}(\mathrm{mJy})$ & $\delta r$ & $F\left(10^{-14} \mathrm{erg} \mathrm{s}^{-1}\right)$ & $\delta r$ \\
\hline 131 & 23.4 & & 1.53 & 0.83 & 1.11 & 1.136 & 2.234 & 0.508 & 15.067 & 0.757 & 6.838 & 0.551 & 28.327 & 0.405 \\
\hline 132 & 23.2326878 & 30.5413206 & .4 & 1.803 & 441 & 0.358 & 27 & 0.443 & 292 & 0.664 & 115 & 0.616 & 17.741 & 0.603 \\
\hline 133 & 23.3254282 & 30.5561522 & -99.0 & -99.0 & 9.0 & -99.0 & 9.0 & -99.0 & -99.0 & -99.0 & 17.98 & 0.014 & -99.0 & -99.0 \\
\hline 134 & 3.5330369 & 30.5465901 & 0.126 & 0.692 & 17 & 0.681 & 0.968 & 0.392 & 56 & 0.684 & 0.8 & 0.101 & 1.53 & 1.739 \\
\hline 135 & 3.2550544 & 30.5461019 & -99.0 & -99.0 & -99.0 & -99.0 & -99.0 & -99.0 & -99.0 & -99.0 & 1.779 & 0.107 & 0.415 & 1.278 \\
\hline 136 & 3.5951658 & 30.5693594 & 0.33 & 0.546 & 0.47 & 0.766 & 2.21 & 0.735 & 3.833 & 0.513 & 43.9 & 1.253 & 2.26 & 0.655 \\
\hline 137 & 23.4600168 & 30.5420632 & 4.37 & 2.58 & 3.46 & 2.355 & 4.68 & 2.141 & 2.214 & 2.13 & 3.91 & 0.026 & 4.857 & 0.455 \\
\hline 138 & 23.3221538 & 30.5362579 & 1.32 & 0.449 & 0.934 & 0.466 & 0.77 & 0.439 & 0.75 & 0.523 & 0.41 & 0.0 & -99.0 & -99.0 \\
\hline 139 & 23.58378 & 30.5509049 & -99.0 & -99.0 & -99.0 & -99.0 & -99.0 & -99.0 & 13.8 & 0.907 & 6.49 & 0.033 & -99.0 & -99.0 \\
\hline 140 & 23.6781752 & 30.5426413 & 0.116 & 1.248 & 0.173 & 1.347 & 0.369 & 0.767 & 0.868 & 0.823 & 1.241 & 0.123 & -99.0 & -99.0 \\
\hline 141 & 3.2514388 & 30.5408204 & 0.21 & 1.598 & -99.0 & -99.0 & 1.79 & 0.267 & 10.33 & 0.771 & 1. & 0.001 & -99.0 & -99.0 \\
\hline 142 & 3.2380036 & 30.5408871 & -99.0 & -99.0 & -99.0 & -99.0 & -99.0 & -99.0 & 0.467 & 1.858 & 0. & 0.001 & -99.0 & -99.0 \\
\hline 143 & 3.3351058 & .5480067 & 0.16 & 1.257 & 0.06 & 1.493 & 3.5 & 1.726 & 3 & 1.283 & & 0.001 & 62 & 1.985 \\
\hline 144 & 3.4131379 & 30.6354521 & 0.245 & 1.131 & 0.57 & 1.02 & 1.519 & 1.131 & 3.996 & 1.327 & 33.199 & 1.682 & 1.21 & 1.508 \\
\hline 145 & 3.2365346 & 5483727 & 0.51 & 1.229 & 49 & 1.106 & 1.13 & 1.16 & -99.0 & -99.0 & & 0.028 & 238 & 0.801 \\
\hline 146 & 3.3896177 & 5597423 & -99.0 & -99.0 & 9.0 & -99.0 & & 2.283 & 2.16 & 0.405 & & 0.001 & .63 & 1.289 \\
\hline 147 & 23.6815822 & .5475032 & 0.019 & 0.262 & 14 & 0.666 & 12 & 0.448 & 20 & 0.381 & & 0.001 & -99.0 & -99.0 \\
\hline 148 & 23.660053 & 7418 & -99.0 & -99.0 & 022 & .855 & 46 & 0.78 & 0.24 & 0.765 & 0. & 0.0 & 1.08 & 0.282 \\
\hline 149 & 23.5934988 & 30.5555251 & 0.35 & 2.284 & 0.19 & 2.347 & -99.0 & -99.0 & 1.3 & 2.444 & 5.0 & 0.001 & 10.614 & 0.836 \\
\hline 150 & 3.5349968 & 30.5499343 & -99.0 & -99.0 & 0.075 & 0.888 & 0.388 & 1.102 & 1.327 & 1.09 & 1.883 & 0.863 & -99.0 & -99.0 \\
\hline 151 & 23.7138106 & 30.5466412 & -99.0 & -99.0 & -99.0 & -99.0 & 0.11 & 0.609 & 0.69 & 0.723 & 0.3 & 0.001 & .563 & 1.32 \\
\hline 152 & 23.3790765 & 30.55941 & -99.0 & -99.0 & 0.15 & 1.976 & 1.86 & 2.021 & 10.23 & 1.952 & 9.73 & 0.014 & 9.34 & 1.787 \\
\hline 153 & 23.6028416 & 30.5518649 & 0.18 & 0.737 & 0.12 & 0.801 & 0.111 & 0.7 & 0.173 & 0.495 & 2.72 & 0.002 & .701 & 0.4 \\
\hline 154 & 23.216564 & 30.5486997 & -99.0 & -99.0 & -99.0 & -99.0 & 0.207 & 0.634 & 0.26 & 0.966 & 0.21 & 0.191 & -99.0 & -99.0 \\
\hline 155 & 23.2525742 & 30.5664134 & -99.0 & -99.0 & -99.0 & -99.0 & 5. & 1.867 & 22.98 & 1.005 & 12.75 & 0.618 & 109.91 & 1.795 \\
\hline 156 & 3.4787314 & 30.5667815 & -99.0 & -99.0 & -99.0 & -99.0 & -99.0 & -99.0 & -99.0 & -99.0 & 70 & 1.938 & -99.0 & -99.0 \\
\hline 157 & 2583902 & .5803033 & 1.79 & 1.774 & 03 & 1.622 & & 2.692 & & 1.56 & & 0.453 & & 0.664 \\
\hline 158 & $38^{\prime}$ & 6085 & 0.9 & 2.659 & 1 & 2.681 & & 2.615 & & 2.398 & & 0.406 & & 1.594 \\
\hline 159 & 175 & 97 & & 0.946 & & 0.783 & & 0.826 & & 0.629 & & 0.128 & .0 & -99.0 \\
\hline 160 & .2514615 & 372 & 0 & 0.757 & 0 & 0.701 & & 0.707 & & 0.695 & & 0.001 & 26 & 0.49 \\
\hline 161 & 2925744 & 11 & & 1.969 & 1 & 1.991 & & 2.41 & & 2.073 & & 0.124 & & -99.0 \\
\hline 162 & 23.6074399 & 159 & 0.05 & 0.752 & 0 & 0.451 & 0 & 0.591 & 0.3 & 0.351 & & 0.001 & -99.0 & -99.0 \\
\hline 163 & 23.2525361 & 212 & & 2.459 & -99.0 & -99.0 & 39 & 1.077 & 2.977 & 1.039 & 13.33 & 0.429 & 13.304 & 1.826 \\
\hline 164 & 23.7041713 & 30.5580431 & 0 & 0.532 & 0.23 & 0.416 & 0. & 0.385 & 0.2 & 0.622 & 0. & 0.0 & -99.0 & -99.0 \\
\hline 165 & 23.4966018 & 30.5968722 & 9.53 & 1.065 & 6.8 & 1.018 & 24.41 & 1.07 & 24.015 & 0.747 & 89.879 & 0.041 & 59.847 & 0.813 \\
\hline 166 & 23.4772433 & 30.57 & -99.0 & -99.0 & -99.0 & -99.0 & -99.0 & -99.0 & -99.0 & -99.0 & 12.26 & 0.011 & 7.657 & 1.866 \\
\hline 167 & 23.6714293 & 30.5595353 & 0.014 & 0.541 & 0.015 & 0.573 & 0.144 & 0.799 & 0.3 & 0.922 & 0. & 0.001 & .075 & 0.599 \\
\hline 168 & 3970484 & 30.6078945 & 7.15 & 0.111 & 4.01 & 0.35 & 6.802 & 0.84 & 19.633 & 0.828 & 17.062 & 1.374 & 60.9 & 1.895 \\
\hline 169 & 3.3008364 & 30.5746583 & 0 & 0.97 & 0.067 & 1.242 & 1.284 & 1.296 & 3.218 & 1.223 & 4.6 & 0.352 & 99.0 & -99.0 \\
\hline 170 & 6638671 & .5627627 & 0 & .638 & 0. & 0.618 & -99.0 & -99.0 & .86 & 0.513 & 34 & 0.001 & 99.0 & -99.0 \\
\hline 171 & 23.65 & & & 929 & & 329 & & 1.088 & & 1.018 & & 0.003 & & 1.197 \\
\hline 172 & 5 & 96 & -99.0 & -99.0 & -99.0 & -99.0 & & 0.502 & & 0.288 & 15 & 0.454 & 0 & -99.0 \\
\hline 173 & .57 & & -9 & -99.0 & -99 & -99.0 & & -99.0 & & 1.221 & & 0.001 & & 1.264 \\
\hline 174 & 1 & & & 0.758 & & 0.518 & & 0.574 & 9 & 1.784 & 10 & 0.189 & & 0.764 \\
\hline 175 & 4 & & & 97 & & & & 5 & & 1.342 & & 0. & & 2.382 \\
\hline 176 & .4222639 & 62 & & 2.946 & -99.0 & -99.0 & & 0.317 & & 0.775 & 0 & 0.292 & & 0.396 \\
\hline 177 & 4678741 & 30.57 & -99.0 & -99.0 & -99.0 & -99.0 & 0 & 2.329 & 2.425 & 1.738 & 58.26 & 0.721 & 99.0 & -99.0 \\
\hline 178 & 2181368 & 627 & 0 & 1.853 & & 2.035 & 1.8 & 0.451 & & 0.973 & 8.74 & 0.56 & 31 & 2.917 \\
\hline 179 & 3957336 & 30.5732324 & 0.062 & 2.789 & 0.053 & 0.667 & & 0.592 & 0.557 & 0.413 & 0.91 & 0.061 & 99.0 & -99.0 \\
\hline 180 & 3483409 & 30.5743059 & 0 & 0.881 & 0.31 & 0.659 & 0.188 & 0.463 & 0.67 & 0.402 & 1.66 & 0.038 & -99.0 & -99.0 \\
\hline 181 & 23.2877777 & 30.5827724 & 0 & 2.588 & 0.13 & 2.173 & & 2.023 & 4.12 & 2.096 & 3.74 & 1.764 & -99.0 & -99.0 \\
\hline 182 & 3.3859728 & 30.6155237 & 0.81 & 1.825 & 1 & 1.776 & 11 & 1.326 & 4.592 & 0.667 & 35.84 & 0.064 & 9.0 & 0.433 \\
\hline 183 & 2423017 & .5802227 & -99.0 & -99.0 & -99.0 & -99.0 & 0.215 & 1.322 & 0. & 1.471 & 11.369 & 1.11 & -99.0 & -99.0 \\
\hline 184 & 23.3909423 & 30.6915587 & 24.63 & 2.767 & 23.3 & .096 & -99.0 & -99.0 & -99.0 & -99.0 & 1434.67 & 0.929 & 99.0 & -99.0 \\
\hline 185 & 23.4390916 & 23 & & 0.545 & & 1.017 & & 0.646 & 30.40 & 0.622 & 136.49 & 0.047 & 92 & 0.546 \\
\hline 186 & 23.274171 & 30.5832355 & 0.019 & 2.686 & -99.0 & -99.0 & 0. & 2.668 & 1.651 & 2.699 & 1.76 & 0.022 & -99.0 & -99.0 \\
\hline 187 & 23.56 & & & 0.863 & 2.6 & 1.006 & & 2.571 & 4.592 & 2.638 & 216.15 & 0.426 & 63.908 & 0.404 \\
\hline 188 & 844 & 639 & & 0.3 & & 0.308 & & 0.553 & & 0.295 & 1.1 & 0.052 & -99.0 & -99.0 \\
\hline 189 & 23.3151417 & 30.5 & & 1.589 & & 1.067 & & 0.813 & & 1.513 & 2.4 & 0.264 & & 1.19 \\
\hline 190 & 23.2808383 & & & -99.0 & -99.0 & -99.0 & & 2.953 & & 2.474 & & 0.022 & & 1.134 \\
\hline 191 & 23.404885 & 30.5862258 & -99.0 & -99.0 & & & & 0.437 & 8.176 & 0.316 & 1. & 0.002 & 4.148 & 1.275 \\
\hline 192 & 23.45 & & & 0.3 & & & & 1.445 & & 2.902 & & 0.002 & -99.0 & -99.0 \\
\hline 193 & 3949208 & 2892 & & 1.172 & & 0.829 & 20.17 & 1.772 & 18.729 & 0.788 & 56.527 & 0.121 & 61.5 & 0.969 \\
\hline 194 & 23.5957164 & 30.5871002 & & 2.87 & 0. & 2.883 & & 0.848 & & 0.463 & 1.03 & 0.259 & 2.13 & 1.331 \\
\hline 195 & 23.5453392 & 30.597 & & 0.4 & 1. & 0.662 & & 0.529 & & 0.465 & 19.58 & 0.093 & 11.23 & 2.186 \\
\hline 196 & 23.5929936 & 30.5886504 & -99.0 & -99.0 & -99.0 & -99.0 & -99.0 & -99.0 & -99.0 & -99.0 & 1.721 & 0.074 & -99.0 & -99.0 \\
\hline
\end{tabular}


Table 3. continued.

\begin{tabular}{|c|c|c|c|c|c|c|c|c|c|c|c|c|c|c|}
\hline \multirow[t]{2}{*}{ Num. } & \multirow{2}{*}{$\begin{array}{c}\text { RA (J2000) } \\
\left({ }^{\circ}\right)\end{array}$} & \multirow{2}{*}{$\begin{array}{c}\text { Dec }(\mathrm{J} 2000) \\
\left({ }^{\circ}\right)\end{array}$} & \multicolumn{2}{|r|}{$3.6 \mu \mathrm{m}$} & \multicolumn{2}{|r|}{$4.5 \mu \mathrm{m}$} & \multicolumn{2}{|r|}{ 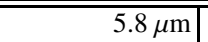 } & \multicolumn{2}{|r|}{$8.0 \mu \mathrm{m}$} & \multicolumn{2}{|r|}{$24 \mu \mathrm{m}$} & & $\overline{\mathrm{H \textrm {H } \alpha}}$ \\
\hline & & & $F_{v}(\mathrm{mJy})$ & $\delta r$ & $F_{v}(\mathrm{mJy})$ & $\delta r$ & $F_{v}(\mathrm{mJy})$ & $\delta r$ & $F_{v}(\mathrm{mJy})$ & $\delta r$ & $F_{v}(\mathrm{mJy})$ & $\delta r$ & $F\left(10^{-14} \mathrm{erg} \mathrm{s}^{-1}\right)$ & $\delta r$ \\
\hline 197 & 23.2319069 & 30.5933869 & 0.24 & \begin{tabular}{l|}
0.252 \\
\end{tabular} & 0.09 & 0.096 & 0.35 & \begin{tabular}{l|l}
0.738 \\
\end{tabular} & 1.48 & 1.431 & 5.63 & 0.016 & 4.73 & 2.611 \\
\hline 198 & 23.50982 & 30.6456077 & 64.19 & 1.915 & -99.0 & -99.0 & -99.0 & -99.0 & 239.2 & 2.931 & 258.82 & 2.863 & 67.405 & 0.8 \\
\hline 199 & 23.4978759 & 30.599838 & 0.67 & 0.361 & 0.686 & 0.388 & 5.627 & 0.23 & 16.026 & 0.234 & 39.301 & 0.292 & -99.0 & -99.0 \\
\hline 200 & 23.6299726 & 30.5893845 & 0.011 & 0.371 & 0.01 & 0.571 & 0.333 & 0.947 & 1.08 & 1.048 & 0.72 & 0.0 & -99.0 & -99.0 \\
\hline 201 & 23.4588671 & 30.6248737 & 2.93 & 0.973 & 2.29 & 1.09 & 16.63 & 1.449 & 28.476 & 0.821 & 100.66 & 1.428 & 39.91 & 2.579 \\
\hline 202 & 23.2861385 & 30.5906052 & 0.98 & 0.598 & 1.23 & 0.714 & 1.47 & 0.577 & 2.03 & 0.596 & 1.25 & 0.003 & -99.0 & -99.0 \\
\hline 203 & 23.4793863 & 30.5914266 & -99.0 & -99.0 & -99.0 & -99.0 & 0.38 & 1.047 & 1.166 & 0.876 & 0.71 & 0.003 & -99.0 & -99.0 \\
\hline 204 & 23.4017975 & 30.5921185 & 4.78 & 0.902 & 2.74 & 0.916 & 2.0 & 0.931 & 1.66 & 0.729 & 0.47 & 0.001 & -99.0 & -99.0 \\
\hline 205 & 23.3237072 & 30.5951935 & 0.2 & 1.553 & 0.09 & 1.175 & 0.57 & 0.185 & 4.1 & 0.362 & 0.23 & 0.171 & 0.36 & 1.812 \\
\hline 206 & 23.4515238 & 30.5964118 & -99.0 & -99.0 & -99.0 & -99.0 & -99.0 & -99.0 & -99.0 & -99.0 & 0.58 & 0.006 & -99.0 & -99.0 \\
\hline 207 & 23.2377615 & 30.5997876 & -99.0 & -99.0 & -99.0 & -99.0 & 0.372 & 0.365 & 1.92 & 0.707 & 1.04 & 0.001 & 12.01 & 2.376 \\
\hline 208 & 23.3562276 & 30.5978429 & 0.77 & 0.492 & 1.28 & 0.508 & 1.54 & 0.463 & 1.84 & 0.41 & 1.35 & 0.0 & -99.0 & -99.0 \\
\hline 209 & 23.4639265 & 30.5974045 & 0.17 & 1.778 & 0.176 & 1.791 & 0.29 & 0.494 & 1.8 & 0.309 & 0.46 & 0.002 & 1.67 & 0.151 \\
\hline 210 & 23.4319437 & 30.6509415 & -99.0 & -99.0 & -99.0 & -99.0 & -99.0 & -99.0 & -99.0 & -99.0 & 187.31 & 1.931 & 197.57 & 2.463 \\
\hline 211 & 23.2292997 & 30.5976103 & -99.0 & -99.0 & -99.0 & -99.0 & -99.0 & -99.0 & -99.0 & -99.0 & 0.606 & 0.176 & 5.143 & 0.751 \\
\hline 212 & 23.2416602 & 30.6017643 & 1.186 & 1.504 & 0.663 & 1.527 & 0.759 & 1.507 & 1.259 & 1.48 & 1.088 & 0.089 & -99.0 & -99.0 \\
\hline 213 & 23.4722505 & 30.6015189 & -99.0 & -99.0 & -99.0 & -99.0 & 0.22 & 1.041 & 25.631 & 0.368 & 0.92 & 0.081 & -99.0 & -99.0 \\
\hline 214 & 23.2332881 & 30.5998748 & 0.378 & 1.271 & 0.418 & 1.391 & 0.381 & 1.613 & 0.52 & 0.71 & 0.434 & 0.114 & -99.0 & -99.0 \\
\hline 215 & 23.4775595 & 30.6015451 & 0.05 & 2.912 & 0.093 & 2.704 & 0.468 & 2.795 & -99.0 & -99.0 & 1.091 & 0.038 & -99.0 & -99.0 \\
\hline 216 & 23.4666875 & 30.611183 & -99.0 & -99.0 & -99.0 & -99.0 & -99.0 & -99.0 & -99.0 & -99.0 & 4.09 & 0.004 & 6.49 & 0.743 \\
\hline 217 & 23.3404764 & 30.6065863 & 0.85 & 0.669 & 0.99 & 0.742 & 1.52 & 0.525 & 2.85 & 0.63 & 3.99 & 0.015 & -99.0 & -99.0 \\
\hline 218 & 23.2833369 & 30.6042448 & -99.0 & -99.0 & -99.0 & -99.0 & 0.066 & 0.28 & 0.509 & 2.497 & 0.21 & 0.002 & -99.0 & -99.0 \\
\hline 219 & 23.5263608 & 30.6283346 & -99.0 & -99.0 & -99.0 & -99.0 & 7.22 & 1.61 & -99.0 & -99.0 & 12.92 & 0.827 & -99.0 & -99.0 \\
\hline 220 & 23.6274042 & 30.6098987 & 0.33 & 1.747 & 0.192 & 0.525 & 0.47 & 0.639 & 1.49 & 0.785 & 2.16 & 0.001 & 0.252 & 0.225 \\
\hline 221 & 23.3639789 & 30.6081481 & 0.17 & 1.616 & 0.105 & 2.48 & 0.3 & 0.595 & 2.415 & 0.544 & 0.75 & 0.002 & 0.047 & 2.58 \\
\hline 222 & 23.5300461 & 30.60892 & 0.43 & 1.354 & 0.12 & 2.643 & 1.849 & 0.813 & 2.59 & 1.838 & 0.99 & 0.003 & 7.11 & 1.103 \\
\hline 223 & 23.4475332 & 30.6187409 & -99.0 & -99.0 & -99.0 & -99.0 & 3.11 & 0.555 & 3.388 & 2.834 & 5.22 & 0.003 & 0.967 & 1.79 \\
\hline 224 & 23.3134189 & 30.6111006 & 0.16 & 2.218 & -99.0 & -99.0 & 0.78 & 0.779 & 12.37 & 1.73 & 1.15 & 0.0 & 0.161 & 0.419 \\
\hline 225 & 23.3702028 & 30.6733838 & 4.117 & 0.621 & 4.589 & 0.608 & 36.54 & 1.197 & 65.556 & 0.527 & 633.39 & 0.017 & 158.22 & 2.251 \\
\hline 226 & 23.50066 & 30.67 & .39 & 0.682 & 5.146 & 0.663 & 26.189 & 0.641 & 76.676 & 0.479 & 1000.35 & 0.274 & 18.689 & 0.531 \\
\hline 227 & 23.4 & 30.6 & 1.11 & 2.732 & -99.0 & -99.0 & 7.61 & 2.254 & -99.0 & -99.0 & 8 & 0.004 & -99.0 & -99.0 \\
\hline 228 & 23.24 & 30.6 & 0.09 & 0.502 & 0.03 & 0.437 & 0.54 & 0.516 & 3.48 & 0.623 & 0.97 & 0.001 & 1.09 & 0.93 \\
\hline 229 & 23.5 & 30.6 & 1.17 & 0.742 & 2.05 & 0.488 & 10.17 & 0.908 & 7.407 & 0.734 & 21.243 & 0.038 & -99.0 & -99.0 \\
\hline 230 & 23.5074748 & 30.6226856 & -99.0 & -99.0 & 0.23 & 2.296 & 3.17 & 2.106 & -99.0 & -99.0 & 6.56 & 0.001 & 9.177 & 1.012 \\
\hline 231 & 23.306399 & 30.6582237 & 0.84 & 0.928 & 0.66 & 1.451 & 8.71 & 1.534 & 7.003 & 1.096 & 34.39 & 0.035 & 3.433 & 0.507 \\
\hline 232 & 23.3802176 & 30.6340207 & 0.45 & 1.462 & 0.27 & 1.313 & 11.15 & 0.514 & 2.329 & 0.944 & 27.57 & 0.007 & 22.82 & 1.972 \\
\hline 233 & 23.2292626 & 30.61458 & 0.52 & 1.111 & 0.34 & 0.844 & 0.26 & 0.847 & 1.26 & 1.02 & 0.74 & 0.002 & 3.815 & 2.972 \\
\hline 234 & 23.3977557 & 30.6581247 & 4.06 & 0.501 & 3.11 & 0.338 & 9.788 & 0.678 & 27.048 & 0.422 & 194.41 & 0.081 & 18.056 & 0.777 \\
\hline 235 & 23.5122553 & 30.6286521 & 0.91 & 2.567 & 0.7 & 1.64 & 4.26 & 0.763 & 7.66 & 1.218 & 5.76 & 0.002 & 11.722 & 2.571 \\
\hline 236 & 23.2987395 & 30.6491089 & 1.69 & 0.713 & 1.208 & 1.39 & 9.21 & 1.068 & 31.79 & 1.332 & 27.994 & 0.161 & 41.971 & 0.319 \\
\hline 237 & 23.7037368 & 30.6246831 & -99.0 & -99.0 & -99.0 & -99.0 & 0.18 & 2.776 & 0.86 & 2.633 & 3.19 & 0.379 & 0.43 & 1.646 \\
\hline 238 & 23.1891098 & 30.648771 & -99.0 & -99.0 & -99.0 & -99.0 & -99.0 & -99.0 & -99.0 & -99.0 & 61.68 & 1.53 & 368.95 & 1.037 \\
\hline 239 & 23.4483822 & 30.6444345 & 0.669 & 2.756 & 0.606 & 2.751 & 6.135 & 2.951 & 16.634 & 2.544 & 82.36 & 0.453 & 124.33 & 0.148 \\
\hline 240 & 23.53 & 30.6 & -99.0 & -99.0 & 1.23 & 0.464 & 8.07 & 0.492 & 24.91 & 1.143 & 81.69 & 0.036 & -99.0 & -99.0 \\
\hline 241 & 23.27 & 30. & 0.06 & 1.39 & 0.055 & 1.33 & 3 & 1.478 & 2.07 & 1.113 & 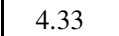 & 0.023 & -99.0 & -99.0 \\
\hline 242 & 23.58 & 30.6 & 0.18 & 1.909 & 0.035 & 1.001 & -99.0 & -99.0 & 14.94 & 0.506 & 4.8 & 0.001 & 20.11 & 1.336 \\
\hline 243 & 23.5361604 & 30.6 & -99.0 & -99.0 & -99.0 & -99.0 & -99.0 & -99.0 & 6.712 & 1.728 & 1.38 & 0.003 & -99.0 & -99.0 \\
\hline 244 & 23.6966122 & 30.6 & 0.3 & 0.355 & 017 & 0.446 & 1.23 & 0.477 & 5.31 & 0.658 & 5.09 & 0.069 & 4.57 & 0.366 \\
\hline 245 & 23.2178479 & 30.6211361 & 0.08 & 1.645 & -99.0 & -99.0 & 0.206 & 0.851 & 0.66 & 0.661 & 0.18 & 0.001 & 1.34 & 0.353 \\
\hline 246 & 23.5156327 & 30.6313122 & 1.251 & 0.883 & 1.145 & 0.816 & 0.907 & 0.814 & 0.818 & 0.821 & 1.562 & 0.084 & -99.0 & -99.0 \\
\hline 247 & 23.5022229 & 30.6357354 & -99.0 & -99.0 & -99.0 & -99.0 & 9.34 & 1.734 & 31.27 & 1.492 & 8.899 & 0.061 & 17.95 & 0.153 \\
\hline 248 & 23.4862749 & 30.6250972 & 0.57 & 1.484 & 0.46 & 1.412 & 1.277 & 1.475 & 4.051 & 2.724 & 1.304 & 0.441 & -99.0 & -99.0 \\
\hline 249 & 23.3670659 & 30.6257606 & 0.15 & 2.989 & 0.1 & 2.719 & -99.0 & -99.0 & -99.0 & -99.0 & 0.39 & 0.003 & 0.055 & 1.631 \\
\hline 250 & 23.5408285 & 30.6375233 & 1.01 & 0.862 & 1.5 & 0.802 & 3.05 & 0.799 & 8.87 & 0.26 & 11.76 & 0.01 & 0.2 & 0.412 \\
\hline 251 & 23.3032423 & 30.6451688 & 3.86 & 2.367 & 3.09 & 2.304 & 2.518 & 1.353 & 17.14 & 2.974 & 35.19 & 0.261 & 14.732 & 0.688 \\
\hline 252 & 23.3588787 & 30.6304911 & -99.0 & -99.0 & -99.0 & -99.0 & 5.559 & 1.418 & 1.611 & 2.255 & 0.74 & 0.073 & -99.0 & -99.0 \\
\hline 253 & 23.3605019 & 30.6506041 & 2.03 & 2.931 & -99.0 & -99.0 & -99.0 & -99.0 & 0.727 & 1.155 & 17.68 & 0.564 & 6.937 & 1.766 \\
\hline 254 & 23.3648166 & 30.6287867 & -99.0 & -99.0 & -99.0 & -99.0 & 1.039 & 0.337 & 2.472 & 0.719 & 1.276 & 0.147 & -99.0 & -99.0 \\
\hline 255 & 23.6939075 & 30.637165 & 0.31 & 0.577 & 0.12 & 0.684 & 1.28 & 0.681 & 2.748 & 0.072 & 2.226 & 0.126 & 4.922 & 0.723 \\
\hline 256 & 23.455278 & 30.6333584 & -99.0 & -99.0 & 0.32 & 0.549 & 1.45 & 1.161 & 4.75 & 0.753 & 4.55 & 0.019 & -99.0 & -99.0 \\
\hline 257 & 23.3618237 & 30.6449707 & 1.34 & 1.011 & 0.64 & 0.771 & 2.126 & 0.487 & 6.18 & 0.2 & 17.28 & 0.38 & 13.11 & 1.429 \\
\hline 258 & 23.5539412 & 30.6350589 & 0.45 & 0.841 & 0.83 & 0.636 & 1.2 & 0.544 & 1.09 & 0.546 & 1.39 & 0.046 & -99.0 & -99.0 \\
\hline 259 & 23.5738589 & 30.634089 & 0.5 & 1.565 & 0.22 & 1.541 & -99.0 & -99.0 & -99.0 & -99.0 & 0.59 & 0.003 & -99.0 & -99.0 \\
\hline 260 & 23.2934401 & 30.6466653 & 0.46 & 0.329 & 0.73 & 0.235 & 0.87 & 0.306 & 2.45 & 0.463 & 11.18 & 0.055 & -99.0 & -99.0 \\
\hline 261 & 23.5768249 & 30.6354645 & 1.881 & 1.239 & 1.08 & 1.183 & 0.947 & 1.147 & 0.983 & 1.166 & 0.487 & 0.255 & -99.0 & -99.0 \\
\hline 262 & 23.3230689 & 30.6363488 & 0.28 & 0.687 & 0.23 & 0.625 & 0.206 & 0.759 & 0.94 & 0.715 & 1.31 & 0.001 & -99.0 & -99.0 \\
\hline
\end{tabular}


Table 3. continued.

\begin{tabular}{|c|c|c|c|c|c|c|c|c|c|c|c|c|c|c|}
\hline \multirow[t]{2}{*}{$\overline{\text { Num. }}$} & \multirow{2}{*}{$\begin{array}{c}\text { RA (J2000) } \\
\left({ }^{\circ}\right)\end{array}$} & \multirow{2}{*}{$\begin{array}{c}\text { Dec (J2000) } \\
\left(^{\circ}\right) \\
\end{array}$} & \multicolumn{2}{|r|}{$3.6 \mu \mathrm{m}$} & \multicolumn{2}{|r|}{$4.5 \mu \mathrm{m}$} & \multicolumn{2}{|r|}{$\overline{5.8 \mu \mathrm{m}}$} & \multicolumn{2}{|r|}{$8.0 \mu \mathrm{m}$} & \multicolumn{2}{|r|}{$24 \mu \mathrm{m}$} & & $\overline{\mathrm{H} \alpha}$ \\
\hline & & & $F_{v}(\mathrm{mJy})$ & $\delta r$ & $F_{v}(\mathrm{mJy})$ & $\delta r$ & $F_{v}(\mathrm{mJy})$ & $\delta r$ & $F_{V}(\mathrm{mJy})$ & $\delta r$ & $F_{v}(\mathrm{mJy})$ & $\delta r$ & $F\left(10^{-14} \mathrm{erg} \mathrm{s}^{-1}\right)$ & $\delta r$ \\
\hline 263 & 23.2229493 & & 0.289 & 0.495 & 0.346 & 0.568 & 0.423 & 0.645 & -99.0 & -99.0 & 2.639 & 1.203 & -99.0 & $\begin{array}{l}-99.0 \\
\end{array}$ \\
\hline 264 & 23.2215632 & 30.6358148 & & 0.291 & & 0.267 & 9.0 & -99.0 & 0.4 & 0.262 & 0.79 & 0.181 & -99.0 & -99.0 \\
\hline 265 & 23.6530394 & 30.6374993 & .0 & -99.0 & 9.0 & -99.0 & 12 & 2.684 & -99.0 & -99.0 & 1.0 & 0.071 & 1.01 & 2.789 \\
\hline 266 & 3.5325477 & 30.653787 & 0.343 & 0.392 & 339 & 0.217 & 436 & 0.274 & 6.768 & 0.151 & 14.889 & 0.147 & -99.0 & -99.0 \\
\hline 267 & 3.4320263 & 30.6833536 & 5.4 & 2.729 & 25 & 2.228 & 41.51 & 1.861 & 7.808 & 1.384 & 46.14 & 0.145 & 13.007 & 1.355 \\
\hline 268 & 3.3737535 & 30.6429053 & -99.0 & -99.0 & 0.033 & 1.193 & 0.44 & 1.176 & 5.924 & 0.886 & 0.95 & 0.063 & -99.0 & -99.0 \\
\hline 269 & 3.4835742 & 30.6530796 & -99.0 & -99.0 & -99.0 & -99.0 & -99.0 & -99.0 & 1.722 & 2.778 & 4.2 & 0.011 & -99.0 & -99.0 \\
\hline 270 & 3.3420438 & 30.6461182 & 0.41 & 0.257 & 0.82 & 0.826 & 1.69 & 0.984 & 3.53 & 0.985 & 1.91 & 0.003 & -99.0 & -99.0 \\
\hline 271 & 3.5435613 & 30.6544413 & -99.0 & -99.0 & 0.143 & 0.909 & 6.21 & 2.38 & 9.295 & 2.452 & 9.04 & 0.115 & 11.267 & 0.185 \\
\hline 272 & 23.3503725 & 30.6532 & -99.0 & -99.0 & -99.0 & -99.0 & -99.0 & -99.0 & 2.279 & 2.06 & 0.46 & 1.056 & -99.0 & -99.0 \\
\hline 273 & 23.407445 & 30.6596327 & 0.274 & 0.567 & 0.23 & 0.562 & 2.401 & 0.863 & 6.703 & 0.499 & 6.978 & 0.039 & .566 & 0.279 \\
\hline 274 & 23.3403854 & 30.6495644 & 0.673 & 0.431 & 0.556 & 0.36 & 0.708 & 0.383 & 2.172 & 0.565 & 2.416 & 0.204 & -99.0 & -99.0 \\
\hline 275 & 23.4880319 & 30.6493804 & -99.0 & -99.0 & -99.0 & -99.0 & & 0.433 & 4. & 1.053 & & 0.121 & -99.0 & -99.0 \\
\hline 276 & 3.4900755 & 30.7053428 & 1.925 & 0.568 & 8 & 1.248 & 25.06 & 1.298 & 37.937 & 0.372 & 95.97 & 0.085 & 0.55 & 1.81 \\
\hline 277 & 3.6492502 & 30.6738673 & 0. & 1.872 & -99.0 & -99.0 & & 0.621 & 3.133 & 1.089 & 15.21 & 1.223 & .361 & 0.694 \\
\hline 278 & 3.2647003 & 6511552 & & 0.52 & & 0.57 & & 0.617 & 81 & 0.746 & 91 & 0.035 & 99.0 & -99.0 \\
\hline 279 & 3.3465799 & 6508679 & -99.0 & -99.0 & -99.0 & -99.0 & & 0.556 & 5. & 0.768 & 1. & 0.191 & 99.0 & -99.0 \\
\hline 280 & 3.4057678 & 30.6574906 & 0.61 & 2.36 & 0. & 1.523 & & 0.848 & -99.0 & -99.0 & 10.303 & 0.973 & 99.0 & -99.0 \\
\hline 281 & 23.6559155 & 30.670175 & -99.0 & -99.0 & 0.024 & 0.665 & 0.075 & 1.281 & -99.0 & -99.0 & 1.94 & 0.362 & .084 & 0.677 \\
\hline 282 & 3.3305157 & 30.6582338 & -99.0 & -99.0 & -99.0 & -99.0 & -99.0 & -99.0 & 9.32 & 1.776 & 2.16 & 0.049 & .594 & 2.247 \\
\hline 283 & 23.4265111 & 30.6685087 & -99.0 & -99.0 & -99.0 & -99.0 & 4.62 & 2.732 & -99.0 & -99.0 & 6.24 & 0.001 & -99.0 & -99.0 \\
\hline 284 & 23.2787484 & 30.6528663 & 0.2 & 0.405 & 0.13 & 0.251 & 0.232 & 0.62 & 0.235 & 0.639 & 0.88 & 0.001 & -99.0 & -99.0 \\
\hline 285 & 23.4972031 & 30.6574364 & 0.131 & 2.03 & 0.108 & 1.824 & 1.02 & 0.872 & 6.824 & 0.445 & 0.95 & 0.003 & -99.0 & -99.0 \\
\hline 286 & 23.422845 & 30.6964213 & 0.622 & 2.564 & 24 & 0.952 & -99.0 & -99.0 & -99.0 & -99.0 & 104.03 & 1.32 & 155.37 & 1.731 \\
\hline 287 & 23.5542603 & 30.7003956 & 0.56 & 2.609 & 0.37 & 2.637 & -99.0 & -99.0 & 99.52 & 2.491 & 36.19 & 0.252 & 5.406 & 2.558 \\
\hline 288 & 3.5534039 & 30.6712449 & & 2.402 & & 2.409 & -99.0 & -99.0 & -99.0 & -99.0 & 565 & 0.014 & 8.56 & 1.102 \\
\hline 289 & 3.6025439 & 30.6626101 & 9 & 2.229 & & 2.093 & & 1.855 & -99.0 & -99.0 & & 0.039 & -99.0 & -99.0 \\
\hline 290 & 26 & 077 & & 2.258 & & 1.927 & & 1.624 & & 0.763 & & 0.021 & 7.953 & 2.37 \\
\hline 291 & 2309423 & 021 & & 1.376 & & 1.053 & & 0.728 & & 1.509 & & 0.001 & 3.93 & 0.897 \\
\hline 292 & 1212 & 7624 & 1.7 & 0.977 & & 0.564 & & 0.049 & 13.102 & 0.571 & 8.785 & 0.274 & .81 & 0.048 \\
\hline 293 & 6956205 & 853 & -99.0 & -99.0 & & -99.0 & & -99.0 & 0. & 1.867 & 0.7 & 0.03 & 99.0 & -99.0 \\
\hline 294 & 23.1957872 & 30.6 & 0.06 & 1.57 & 0.111 & 0.932 & 0.179 & 0.517 & 0.35 & 0.569 & 1 & 0.037 & -99.0 & -99.0 \\
\hline 295 & 23.4160083 & 30.6686131 & -99.0 & -99.0 & .0 & -99.0 & & 2.184 & -99.0 & -99.0 & 3.2 & 0.003 & 1.44 & 1.868 \\
\hline 296 & 23.2580642 & 30.6641098 & 1. & 2.836 & -99.0 & -99.0 & 1. & 1.125 & 4.43 & 1.691 & 2.76 & 0.254 & 0.4 & 2.236 \\
\hline 297 & 3.4612661 & 30.6889857 & 15.6 & 1.695 & -99.0 & -99.0 & 4.373 & 0.343 & 13.748 & 0.481 & 21.454 & 0.342 & -99.0 & -99.0 \\
\hline 298 & 3.5288203 & 30.6961545 & 1.91 & 2.232 & 1.41 & 1.704 & 27.88 & 2.015 & -99.0 & -99.0 & 79.67 & 1.85 & 52.0 & 2.093 \\
\hline 299 & 23.3258953 & 30.6893883 & 0.42 & 1.429 & 0.15 & 0.614 & 0.997 & 0.477 & 3.215 & 0.65 & 13.92 & 0.022 & 9.43 & 1.883 \\
\hline 300 & 4075672 & 6798624 & -99.0 & -99.0 & -99.0 & -99.0 & 2 & 1.851 & 66.526 & 0.536 & 4.4 & 0.001 & -99.0 & -99.0 \\
\hline 301 & 3.6259803 & 30.6768091 & 1.99 & 2.837 & 126 & 2.575 & & 2.158 & 2.84 & 1.416 & 4.09 & 0.025 & 2.33 & 1.521 \\
\hline 302 & 23.3743258 & 30.6801545 & 0.558 & 0.672 & & 1.07 & 2.977 & 0.95 & 7.792 & 0.67 & 26.316 & 0.026 & 1.407 & 0.903 \\
\hline 303 & 767 & 85 & & 1.637 & & 34 & & 0.495 & & 0.726 & & 053 & 99.0 & -99.0 \\
\hline 304 & 67 & 3 & & 1.23 & & 0.907 & & 0.602 & & 0.434 & & 0.239 & .0 & -99.0 \\
\hline 305 & 1 & & & 1.163 & & 28 & & 0.893 & & 0.558 & 16. & 0.11 & & 1.116 \\
\hline 306 & 7 & 57 & -99.0 & -99.0 & & 0.541 & 0.2 & 0.505 & & 0.518 & 2. & 0.036 & 99.0 & -99.0 \\
\hline 307 & 8 & 16 & & 2. & & 02 & & 53 & & 0.829 & & 0.0 & -99 & -99.0 \\
\hline 308 & .1794239 & 77 & & 95 & & 96 & & 0.613 & & 0.853 & & 0.129 & 4. & 1.418 \\
\hline 309 & 23.5742394 & 30.6763032 & -99.0 & -99.0 & 0 & -99.0 & -99.0 & -99.0 & -99.0 & -99.0 & 0. & 0.004 & 0.0 & 2.943 \\
\hline 310 & .1765675 & 30.6 & -99.0 & -99.0 & -99.0 & -99.0 & -99.0 & -99.0 & -99.0 & -99.0 & 1.346 & 1.003 & -99.0 & -99.0 \\
\hline 311 & 3965969 & 30.6775016 & 0 & 1.057 & 0 & 1.017 & 0. & 1.198 & 1 & 0.749 & 0.77 & 0.066 & -99.0 & -99.0 \\
\hline 312 & 23.6407516 & 30.6849669 & & 1.025 & & 0.963 & 1.65 & 0.948 & 3.2 & 0.972 & 7.06 & 0.029 & 3.23 & 0.946 \\
\hline 313 & 23.4473536 & 30.688507 & 0.135 & 2.195 & & 2.115 & 2.25 & 2.463 & 1.595 & 2.688 & 3.83 & 2.687 & -99.0 & -99.0 \\
\hline 314 & 23.2617399 & 30.6859556 & 0 & 1.885 & 244 & 2.032 & 0. & 1.319 & 3.16 & 1.468 & 2.054 & 1.499 & 7.229 & 0.812 \\
\hline 315 & .7281912 & 6883464 & -99.0 & -99.0 & -99.0 & -99.0 & 0.204 & 0.831 & .84 & 0.886 & 1.4 & 0.108 & 323 & 0.012 \\
\hline 316 & 23.6269102 & 30.6896408 & -99.0 & -99.0 & 0 & 2.926 & 18 & 1.639 & 0.1 & 1.395 & 7.72 & 0.001 & 11.32 & 2.609 \\
\hline 317 & 2460603 & 6929722 & & 0.666 & & 0.69 & & 0.243 & 9.5 & .81 & 12.61 & 0.0 & 15 & 2.593 \\
\hline 318 & 23.2774538 & 30.68 & 0.057 & 1.844 & 0.04 & 1.479 & 0.051 & 0.155 & 0.199 & 0.445 & 0.53 & 0.001 & -99.0 & -99.0 \\
\hline 319 & 985 & 3 & & 0.451 & 0.441 & 0.227 & & 1.065 & 4.618 & 1.032 & 3.664 & 0.673 & 13.348 & 0.68 \\
\hline 320 & 23.2564197 & & & 2.359 & & 2.329 & & 0.52 & 1.6 & 0.648 & & 0.075 & 0.6 & 1.454 \\
\hline 321 & 23.418 & & -99.0 & -99.0 & & 2.713 & 44. & 1.022 & 19.962 & 2.945 & 101.03 & 0.004 & 34.22 & 1.035 \\
\hline 322 & 87 & & & 93 & & 0.479 & & 0.4 & 1.854 & 0.385 & & 0.001 & -99.0 & -99.0 \\
\hline 323 & 23.5191676 & 30.6 & -99.0 & -99.0 & -99.0 & -99.0 & & 1.435 & 3.4 & 0.538 & 1.44 & 0.004 & 1.03 & 0.695 \\
\hline 324 & 23.3100024 & 30.7 & & 0.721 & & 1.019 & 4.867 & 0.963 & 2.831 & 0.448 & 60.1 & 0.513 & -99.0 & -99.0 \\
\hline 325 & .4830367 & 30.7221122 & & 0.5 & & 0. & & 0.582 & 29.56 & 2.521 & 7.342 & 0.299 & 5.1 & 0.262 \\
\hline 326 & 23.5563729 & 30.6925001 & & 1.403 & & 1.542 & 0.097 & 0.815 & 8.933 & 2.896 & 0.66 & 0.004 & 0.234 & 0.635 \\
\hline 327 & 23.2018147 & 30.692144 & -99 & -99.0 & & 89 & 0.2 & 0.6 & & 0.671 & 0.8 & 0.001 & 0.137 & 0.39 \\
\hline 328 & 23.191209 & 30.6932802 & 0.12 & 0.973 & 0.08 & 0.855 & 0.13 & 0.762 & 0.85 & 0.733 & 1.05 & 0.001 & 4.6 & 0.153 \\
\hline
\end{tabular}


Table 3. continued.

\begin{tabular}{|c|c|c|c|c|c|c|c|c|c|c|c|c|c|c|}
\hline \multirow[t]{2}{*}{ Num. } & \multirow{2}{*}{$\begin{array}{c}\text { RA (J2000) } \\
\left({ }^{\circ}\right) \\
\end{array}$} & \multirow{2}{*}{$\begin{array}{c}\text { Dec }(\mathrm{J} 2000) \\
\left({ }^{\circ}\right)\end{array}$} & \multicolumn{2}{|r|}{$3.6 \mu \mathrm{m}$} & \multicolumn{2}{|r|}{$4.5 \mu \mathrm{m}$} & \multicolumn{2}{|r|}{$\overline{5.8 \mu \mathrm{m}}$} & \multicolumn{2}{|r|}{$8.0 \mu \mathrm{m}$} & \multicolumn{2}{|r|}{$24 \mu \mathrm{m}$} & & $\overline{\mathrm{H} \alpha}$ \\
\hline & & & $F_{v}(\mathrm{mJy})$ & $\partial r$ & $F_{v}(\mathrm{mJy})$ & $\delta r$ & $F_{v}(\mathrm{mJy})$ & $\delta r$ & $F_{v}(\mathrm{mJy})$ & $\delta r$ & $F_{v}(\mathrm{mJy})$ & $\delta r$ & $F\left(10^{-14} \mathrm{erg} \mathrm{s}^{-1}\right)$ & $\delta r$ \\
\hline 329 & 23.4626802 & 30.6976633 & 0.19 & 0.924 & 0.16 & 0.515 & 0.33 & 0.531 & 0.86 & 0.448 & 0.6 & 0.002 & -99.0 & -99.0 \\
\hline 330 & 23.3636271 & 30.6999567 & 56 & 1.614 & 0.21 & 1.272 & 0.36 & 0.965 & 1.33 & 0.648 & 1.58 & 0.001 & -99.0 & -99.0 \\
\hline 331 & 23.1789114 & 30.7000799 & 0.042 & 0.893 & 0.014 & 0.805 & 0.13 & 0.591 & 0.17 & 0.844 & 0.4 & 0.003 & -99.0 & -99.0 \\
\hline 332 & 23.6862291 & 30.7104179 & 9.0 & -99.0 & -99.0 & -99.0 & 1.2 & 2.672 & 6.733 & 1.425 & 3.618 & 0.08 & 3.515 & 0.998 \\
\hline 333 & 23.4186642 & 30.7178943 & .0 & -99.0 & -99.0 & -99.0 & 4.1 & 1.536 & -99.0 & -99.0 & 8.48 & 0.002 & -99.0 & -99.0 \\
\hline 334 & 23.2837586 & .7067748 & 0.36 & 1.598 & 0.18 & 1.471 & 0.914 & 0.077 & 2.361 & 0.237 & 1.324 & 0.065 & 1.33 & 0.557 \\
\hline 335 & .4353561 & 30.7437675 & 35 & 0.886 & 24 & 0.948 & 098 & 0.892 & 22.493 & 0.855 & 204.66 & 0.024 & 89.796 & 1.054 \\
\hline 336 & .4823506 & 30.7140345 & 0.164 & 0.783 & 22 & 1.283 & 15 & 0.746 & 4.54 & 0.767 & 2.396 & 0.09 & 0.612 & 0.273 \\
\hline 337 & 3.3705591 & 30.704 & 83 & 1.637 & 0.44 & 1.499 & 35 & 0.428 & 1.736 & 0.04 & 0.82 & 0.002 & .888 & 0.447 \\
\hline 338 & 23.4410897 & 0.7128436 & -99.0 & -99.0 & -99.0 & -99.0 & -99.0 & -99.0 & 11.37 & 2.857 & 6.81 & 0.002 & 25.84 & 1.447 \\
\hline 339 & 23.6892886 & 735 & 0.17 & 1.396 & 0.09 & 0.948 & 0.55 & 0.926 & 3.54 & 0.721 & 1.63 & 0.069 & 4.47 & 0.643 \\
\hline 340 & 23.4813787 & .7577943 & 8.91 & 1.926 & 6.97 & 1.9 & -99.0 & -99.0 & -99.0 & -99.0 & 200.59 & 0.007 & 263.49 & 1.844 \\
\hline 341 & 23.3779287 & .7073905 & 0.27 & 2.296 & 0.12 & 2.193 & 0.373 & 0.507 & 7.83 & 1.777 & 0.82 & 0.002 & -99.0 & -99.0 \\
\hline 342 & 23.5046251 & 30.7315513 & 2.66 & 1.016 & 1.84 & 1.07 & 13.81 & 0.602 & 50.43 & 0.789 & 58.93 & 0.006 & 35.11 & 0.376 \\
\hline 343 & 23.3284211 & 30.7127914 & 0.302 & 0.827 & 0.56 & 1.439 & 0.53 & 0.953 & 0.53 & 0.715 & 0.829 & 0.031 & -99.0 & -99.0 \\
\hline 344 & 23.6847832 & 261 & 18 & 0.335 & 0.031 & 0.496 & 0.444 & 0.251 & 1.8 & 0.158 & 3.46 & 0.006 & 1.75 & 2.513 \\
\hline 345 & 23.4427305 & 30.7 & 2 & 2.677 & 1.42 & 0.641 & 6.53 & 1.924 & 20.49 & 1.623 & 21.62 & 0.409 & -99.0 & -99.0 \\
\hline 346 & 23.20 & 13 & 27 & 0.769 & 0.14 & 0.762 & 0. & 0.349 & 0. & 0.788 & $0 ?$ & 0.001 & 99.0 & -99.0 \\
\hline 347 & 23.3 & 55 & 07 & 0.748 & 0. & 0.801 & & 0.973 & & 1.127 & 2.7 & 0.029 & 0.764 & 0.755 \\
\hline 348 & 23.25 & & 96 & 0.201 & 2.9 & 0.404 & 21 & 0.789 & 72.6 & 0.832 & & 0.11 & & 0.792 \\
\hline 349 & 7 & & -99.0 & -99.0 & -99.0 & -99.0 & & 0.734 & 2.81 & 0.357 & 1 & 0.077 & & 0.488 \\
\hline 350 & 3 & & 0.25 & 2.342 & 0.12 & 2.401 & 0.193 & 1.779 & 1.2 & 0.736 & 0.77 & 0.111 & 99.0 & -99.0 \\
\hline 351 & 55 & & 0 & 1.498 & 03 & 1.702 & 0.97 & 1.087 & 9. & 1.218 & 1.78 & 0.043 & .759 & 1.157 \\
\hline 352 & 23.61 & 68 & 36 & 0.471 & 1.86 & 0.435 & 2.1 & 0.446 & 2.2 & 0.281 & 2.34 & 0.001 & 99.0 & -99.0 \\
\hline 353 & 23.3367059 & 656 & 0.32 & 0.766 & 0.24 & 0.746 & 0.225 & 0.69 & 0.5 & 0.505 & 1.34 & 0.002 & .053 & 1.389 \\
\hline 354 & 3.3398438 & 363 & -99.0 & -99.0 & 0.128 & 0.745 & 0.096 & 0.944 & 0.212 & 0.553 & 0.759 & 0.08 & -99.0 & -99.0 \\
\hline 355 & 23.4290995 & 30.74 & 2.63 & 1.304 & 1.95 & 1.101 & 7.748 & 1.563 & 23.673 & 1.903 & 49.43 & 0.048 & 38.495 & 0.366 \\
\hline 356 & 23.5378834 & 30.73 & 20 & 0.465 & 0.335 & 0.474 & 0.8 & 0.517 & 1.37 & 0.383 & 2.66 & 0.02 & -99.0 & -99.0 \\
\hline 357 & 23.5755386 & 384 & -99.0 & -99.0 & 0.034 & 0.303 & 0.897 & 1.137 & 2.69 & 0.639 & 0.562 & 0.081 & 99.0 & -99.0 \\
\hline 358 & 23.7 & 576 & 107 & 0.109 & 014 & 0.191 & 0176 & 0.249 & 0.18 & 0.346 & 03 & 0.002 & 99.0 & -99.0 \\
\hline 359 & 23.5783413 & 878 & 9.0 & -99.0 & -99.0 & -99.0 & 1.011 & 0.555 & 2.64 & 0.132 & 1.541 & 0.194 & .291 & 2.661 \\
\hline 360 & 23.50 & 74 & & 2.044 & 063 & 1.276 & & 1.054 & 24 & 1.629 & 34 & 0.011 & 17 & 2.587 \\
\hline 361 & 23.5 & & & & & 0.863 & & 77 & & 0.991 & 17.99 & 0.001 & 15 & 0.92 \\
\hline 362 & 6 & & & 2.333 & & 2.904 & & 33 & 4. & 1.222 & 4.0 & 0.002 & 9.0 & -99.0 \\
\hline 363 & 8 & & & & & 0.427 & & 68 & & 1.068 & 2.0 & 0.001 & 52 & 1.053 \\
\hline 364 & 4 & & & -99.0 & & -99.0 & -99 . & .0 & & 2.5 & 1.319 & 0.027 & & 1.576 \\
\hline 365 & 3 & & .0 & -99.0 & -99.0 & -99.0 & -99.0 & -99.0 & 1.8 & 1.114 & 0. & 0.147 & 99.0 & -99.0 \\
\hline 366 & 23.35 & & -99.0 & -99.0 & -99.0 & -99.0 & 0.96 & 0.248 & 2.885 & 0.444 & 1.67 & 0.092 & 99.0 & -99.0 \\
\hline 367 & 23.5446783 & 336 & -99.0 & -99.0 & -99.0 & -99.0 & 1. & 2.576 & 14.61 & 1.22 & 3.61 & 0.002 & .231 & 1.18 \\
\hline 368 & 23.5526339 & 73 & -99.0 & -99.0 & -99.0 & -99.0 & 1.64 & 1.793 & 9.16 & 0.878 & 2.61 & 0.044 & .821 & 0.745 \\
\hline 369 & 23.5542291 & 305 & -99.0 & -99.0 & -99.0 & -99.0 & -99.0 & -99.0 & 4.463 & 2.273 & 9.31 & 0.181 & -99.0 & -99.0 \\
\hline 370 & 23.2488596 & 536 & -99.0 & -99.0 & -99.0 & -99.0 & 0.135 & 0.596 & 0.31 & 1.048 & 0.39 & 0.001 & -99.0 & -99.0 \\
\hline 371 & 23.266285 & 56 & & 1.412 & & 1.193 & 10 & 0.468 & & 0.639 & 1.3 & 0.001 & -99.0 & -99.0 \\
\hline 372 & 23.192 & 89 & -99.0 & -99.0 & -99.0 & -99.0 & .434 & 0.81 & 0.939 & 0.201 & .21 & 0.174 & 2.75 & 0.775 \\
\hline 373 & 23.3634157 & 251 & & 2.741 & -99.0 & -99.0 & & 0.882 & 7.2 & 1.412 & 9 & 0.15 & 92 & 0.47 \\
\hline 374 & 23.4470914 & & -99.0 & -99.0 & 9.0 & -99.0 & 97 & 2.357 & 33.36 & 2.188 & 5 & 0.028 & 99.0 & -99.0 \\
\hline 375 & 23.2843986 & & & 1.955 & & 0.142 & & 391 & & 0.328 & 0.1 & 0.001 & 243 & 0.824 \\
\hline 376 & 59 & & & -99.0 & & -99.0 & & 0.542 & 0 & 34 & 4 & 0.001 & .0 & -99.0 \\
\hline 377 & & & & -9 & & -99.0 & & & -99.0 & -99.0 & 0.7 & 0.005 & -99.0 & -99.0 \\
\hline 378 & & & & & -9 & -99.0 & -95 & & & & & 0.001 & & 0.47 \\
\hline 379 & 71 & & & 1.068 & & 1.059 & & 0.919 & 2 & 1.2 & 9.9 & 1.357 & 2 & 0.499 \\
\hline 380 & 23.21 & & -99.0 & -99.0 & -99.0 & -99.0 & 0.033 & 0.888 & 0.07 & 0.542 & 0.5 & 0.001 & 0.442 & 2.745 \\
\hline 381 & 23.4686411 & & & 1.3 & 03 & 0 & 2.5 & 1.061 & 21.45 & 1.999 & 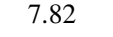 & 0.002 & & 2.665 \\
\hline 382 & 23.280334 & & & 0.478 & & 0.582 & 2 & 0.534 & 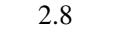 & 0.462 & 0.56 & 0.003 & -99.0 & -99.0 \\
\hline 383 & 23.6238238 & & & 0.542 & & 0.644 & 04 & 0.621 & $0 s$ & 0.62 & 29 & 0.046 & -99.0 & -99.0 \\
\hline 384 & .2076778 & 4 & 0.04 & 0.2 & 0.039 & 0.297 & 0.25 & 0.297 & 0.4 & 0.526 & 0.2 & 0.0 & 746 & 0.123 \\
\hline 385 & 868 & 3 & 0.16 & 1.24 & 00 & 0.962 & 08 & 0.678 & 2.21 & 0.801 & 2.325 & 0.063 & 221 & 0.417 \\
\hline 386 & 23.5321648 & 218 & & 2.541 & & 2.157 & 7 & 1.253 & 2.6 & 1.63 & 0.93 & 0.005 & 121 & 3.0 \\
\hline 387 & 23.3923703 & .7910931 & 0.156 & 0.495 & 0.114 & 0.406 & 1.069 & 0.288 & 3.232 & 0.521 & 1.805 & 0.683 & 0.982 & 0.232 \\
\hline 388 & 23.5520915 & 9057374 & 0.479 & 0.529 & 1.04 & 0.54 & 1.78 & 0.465 & 2.16 & 0.499 & 2.37 & 0.001 & -99.0 & -99.0 \\
\hline 389 & 23.3708825 & .8078404 & -99.0 & -99.0 & -99.0 & -99.0 & 0.38 & 0.869 & 1.37 & 1.661 & 0.39 & 0.001 & 0.19 & 1.104 \\
\hline 390 & 23.4955476 & 162 & 0.1 & 0.637 & 0.14 & 0.559 & 0.71 & 0.645 & 2.32 & 0.641 & 12.55 & 0.0 & 5.44 & 0.429 \\
\hline 391 & 23.3663994 & 3633 & 2.52 & 2.441 & 1.4 & 2.549 & 4.37 & 0.613 & 14.52 & 0.185 & 7.32 & 0.024 & 19.03 & 2.428 \\
\hline 392 & 23.5688082 & 504 & 8.11 & 1.527 & 1.0 & 1.287 & 7.721 & 0.591 & 18.912 & 0.267 & 224.65 & 0.15 & 214.1 & 0.874 \\
\hline 393 & 23.38 & 39 & -99.0 & -99.0 & -99.0 & -99.0 & & 1.192 & & 2.189 & 0.51 & 0.002 & 0.002 & 0.597 \\
\hline 394 & 23.2177947 & 30.8619643 & 0.032 & 0.507 & 0.043 & 0.43 & 0.104 & 0.804 & 0.204 & 0.305 & 0.3 & 0.0 & 0.038 & 0.166 \\
\hline
\end{tabular}


Table 3. continued.

\begin{tabular}{|c|c|c|c|c|c|c|c|c|c|c|c|c|c|c|}
\hline \multirow[t]{2}{*}{ Num. } & \multirow{2}{*}{$\begin{array}{c}\text { RA (J2000) } \\
\left({ }^{\circ}\right) \\
\end{array}$} & \multirow{2}{*}{$\begin{array}{c}\text { Dec (J2000) } \\
\left(^{\circ}\right) \\
\end{array}$} & \multicolumn{2}{|r|}{$3.6 \mu \mathrm{m}$} & \multicolumn{2}{|r|}{$4.5 \mu \mathrm{m}$} & \multicolumn{2}{|r|}{$5.8 \mu \mathrm{m}$} & \multicolumn{2}{|r|}{$8.0 \mu \mathrm{m}$} & \multicolumn{2}{|r|}{$24 \mu \mathrm{m}$} & & $\overline{\mathrm{H} \alpha}$ \\
\hline & & & $F_{v}(\mathrm{mJy})$ & . & $F_{v}(\mathrm{mJy})$ & $\delta r$ & $F_{v}(\mathrm{mJy})$ & $\delta r$ & $F_{v}(\mathrm{mJy})$ & $\delta r$ & $F_{v}(\mathrm{mJy})$ & $\delta r$ & $F\left(10^{-14} \mathrm{erg} \mathrm{s}^{-1}\right)$ & $\delta r$ \\
\hline 395 & 23.378 & & 1.42 & 0.538 & 1.27 & 0.618 & 0.9 & 0.858 & 1.32 & 0.934 & 0.45 & 0.003 & $\begin{array}{l}-99.0 \\
\end{array}$ & -99.0 \\
\hline 396 & 23.51218 & 30.8572721 & .5 & 2.392 & 3 & 0.738 & 57 & 0.313 & 36 & 1.906 & 46 & 0.007 & 50.09 & 0.349 \\
\hline 397 & .4574031 & 30.8614626 & 0.118 & 2.448 & 161 & 1.895 & 5 & 0.764 & 75 & 1.148 & 3 & 0.052 & 11.474 & 0.803 \\
\hline 398 & 3.5458523 & 30.8079254 & 0.11 & 2.147 & 9.0 & -99.0 & -99.0 & -99.0 & -99.0 & -99.0 & 15.57 & 0.848 & -99.0 & -99.0 \\
\hline 399 & 3.6715006 & 30.8513604 & -99.0 & -99.0 & -99.0 & -99.0 & -99.0 & -99.0 & -99.0 & -99.0 & 0.75 & 0.001 & -99.0 & -99.0 \\
\hline 400 & 23.5263124 & 30.8606255 & -99.0 & -99.0 & 0.09 & 2.719 & 1.646 & 1.588 & 3.841 & 0.876 & 5.19 & 0.033 & 8.74 & 2.26 \\
\hline 401 & 23.4338307 & 30.8099453 & 0.42 & 0.377 & 0.22 & 0.316 & 1.81 & 0.49 & 4.591 & 0.373 & 4.91 & 0.02 & 3.16 & 0.142 \\
\hline 402 & 23.55695 & 30.7896318 & 0.54 & 2.435 & 0.11 & 1.751 & 2.128 & 0.767 & 4.974 & 1.253 & 7.48 & 0.144 & 3.002 & 0.59 \\
\hline 403 & 23.4949754 & 30.7908804 & 0.23 & 1.15 & 0.12 & 1.155 & 0.89 & 1.118 & 2.897 & 1.125 & 2.13 & 0.173 & 0.79 & 1.676 \\
\hline 404 & 23.3089648 & 30.8568698 & 0.08 & 0.558 & 0.1 & 0.515 & 0.33 & 0.578 & 1.22 & 0.534 & 2.79 & 0.033 & -99.0 & -99.0 \\
\hline 405 & 23.3987872 & 30.8482912 & 0.418 & 1.341 & 0.334 & 0.872 & 1.296 & 0.526 & 3.325 & 0.286 & 5.256 & 0.048 & 16.669 & 1.013 \\
\hline 406 & 23.6087753 & 30.8166817 & 0.86 & 1.861 & 0.51 & 2.174 & 2.82 & 0.27 & 4.091 & 0.143 & 7.4 & 0.452 & 1.228 & 0.251 \\
\hline 407 & 23.548629 & 0.8132312 & 0 & 1.895 & 0.066 & 2.115 & -9 & -99.0 & -99.0 & -99.0 & & 0.179 & 9.0 & -99.0 \\
\hline 408 & 23.3052668 & 30.8426466 & 0.34 & 1.057 & 0.23 & 0.655 & 1.94 & 0.651 & 7.54 & 1.407 & 6.27 & 0.017 & 3.91 & 0.292 \\
\hline 409 & 3.3952365 & 30.8370405 & 0.24 & 0.936 & 13 & 1.057 & -99.0 & -99.0 & 34 & 2.97 & 9.0 & 0.015 & .1 & 2.414 \\
\hline 410 & 3.4613394 & 30.7949125 & -99.0 & -99.0 & -99.0 & -99.0 & -99.0 & -99.0 & -99.0 & -99.0 & & 0.003 & 9.0 & -99.0 \\
\hline 411 & .4249291 & 30.7931596 & 0.93 & 0.691 & 0.58 & 0.628 & 5 & 0.686 & 5.086 & 1.031 & 15.48 & 0.132 & 64 & 0.312 \\
\hline 412 & 4949753 & 30.8346301 & 36 & 0.562 & 0.32 & 516 & 0 & 0.482 & 1.84 & 0.785 & 1.84 & 0.001 & 14 & 2.053 \\
\hline 413 & 3.3014687 & 30.821464 & 0.14 & 1.16 & -99.0 & -99.0 & 0.36 & 1.139 & 4.6 & 1.768 & 1.30 & 0.019 & 9.0 & -99.0 \\
\hline 414 & 3.2957009 & 30.8095165 & 0.12 & 0.296 & 0.08 & 0.152 & 0.98 & 0.77 & 2.433 & 1.539 & 11.43 & 0.744 & 99.0 & -99.0 \\
\hline 415 & 23.4019926 & 30.8439406 & 0.5 & 1.349 & 0.39 & 1.127 & 1.06 & 1.506 & 4.41 & 1.54 & 7.596 & 0.084 & 0.62 & 1.284 \\
\hline 416 & 23.4841595 & 30.8511127 & 0.21 & 1.516 & 0.129 & 0.961 & 0.31 & 1.15 & 0.913 & 1.057 & 0.34 & 0.002 & 265 & 0.582 \\
\hline 417 & 23.366927 & 30.8427976 & 0.054 & 1.423 & 0.063 & 1.383 & 0.087 & 0.883 & 0.267 & 0.669 & 0.75 & 0.001 & -99.0 & -99.0 \\
\hline 418 & 23.2181081 & 30.8384177 & 0.24 & 1.441 & 0.17 & 0.961 & 0.56 & 0.534 & 1.58 & 0.643 & 7.24 & 0.033 & 1.57 & 0.729 \\
\hline 419 & 23.4492321 & 30.8466044 & 0.52 & 0.091 & 0.34 & 0.399 & 1.0 & 2.326 & -99.0 & -99.0 & 4.9 & 0.042 & 1.62 & 2.827 \\
\hline 420 & 3.5210173 & 30.8166038 & -99.0 & -99.0 & -99.0 & -99.0 & 11 & 1.911 & 4.995 & 0.037 & & 0.129 & 9.0 & -99.0 \\
\hline 421 & 1812805 & 30.8018275 & _99 & -99.0 & 006 & 1.459 & 0.16 & 1.266 & & 1.309 & & 0.033 & 073 & 2.044 \\
\hline 422 & 23.4907269 & 30.7770533 & 0 & -99.0 & -9 & -99.0 & 2.286 & 1.997 & 7. & 2.274 & 1.2 & 0.37 & & 1.111 \\
\hline 423 & .4144609 & 9326 & -99.0 & -99.0 & -99.0 & -99.0 & -9 & -99.0 & -99.0 & -99.0 & & 0.001 & 0 & -99.0 \\
\hline 424 & 6280097 & 8284288 & -99.0 & -99.0 & -9 & -99.0 & & 0.524 & 1 & 0.894 & 1.288 & 0.01 & & 0.124 \\
\hline 425 & 3474003 & 34719 & 0.1 & 1.964 & 0.099 & 1.606 & 0 & 0.921 & 0. & 0.896 & 3. & 0.073 & & 0.475 \\
\hline 426 & 23.4941016 & 5477 & 1. & 0.432 & & 0.432 & 5. & 0.661 & $80 .($ & 1.543 & & 0.115 & & 0.505 \\
\hline 427 & 23.495441 & 7853 & 0.757 & 0.506 & 0.666 & 0.611 & 3.374 & 0.857 & 25.98 & 1.503 & 8.038 & 0.127 & 1 & 0.463 \\
\hline 428 & 3.3024284 & 30.8107841 & 0.15 & 0.722 & -99.0 & -99.0 & 0.14 & 0.798 & 0.2 & 0.727 & 1.636 & 0.097 & 02 & 1.531 \\
\hline 429 & 3.4907454 & 30.8176074 & 3.08 & 1.941 & 2.23 & 2.174 & 13.87 & 1.668 & 11.191 & 0.559 & 19.19 & 0.185 & 11.016 & 0.295 \\
\hline 430 & 23.4626141 & 30.8213831 & -99.0 & -99.0 & 0.088 & 0.45 & 0.146 & 0.787 & 0.168 & 0.628 & 1.197 & 0.029 & 99.0 & -99.0 \\
\hline 431 & 23.4990198 & 30.8227204 & 0.343 & 0.789 & 0.277 & 0.807 & 2.236 & 0.853 & 5.924 & 1.021 & 5.058 & 0.004 & 99.0 & -99.0 \\
\hline 432 & 3.4840875 & 30.824481 & -99.0 & -99.0 & -99.0 & -99.0 & 0.2 & 0.75 & 1.173 & 0.998 & 0 & 0.001 & 25 & 0.68 \\
\hline 433 & 3.3483471 & 30.8253958 & -99.0 & -99.0 & -99.0 & -99.0 & 0.2 & 1.746 & 0.85 & 0.886 & 0. & 0.022 & 99.0 & -99.0 \\
\hline 434 & .5411217 & 30.8183276 & 0.35 & 1.125 & & 1.116 & 0 & 0.539 & 5.944 & 1.288 & 0.947 & 0.296 & 99.0 & -99.0 \\
\hline 435 & 36 & 711 & & 2.647 & -99.0 & -99.0 & 000 & -99.0 & & 1.862 & & 0.027 & & -99.0 \\
\hline 436 & 998 & 63 & & 1.447 & 1. & 1.375 & & 1.399 & 2 & 1.879 & 48 & 0.276 & & 2.509 \\
\hline 437 & 4 & & $0 .($ & 1.288 & & 1.187 & & 0.726 & & 1.013 & & 0.003 & & 0.398 \\
\hline 438 & 9 & & -9 & -99.0 & -99.0 & -99.0 & & 2.567 & 4 & 2.944 & 24 & 0.846 & & -99.0 \\
\hline 439 & 9 & & -9 & -99.0 & & 2.645 & & 1.75 & & 0.879 & & 0.002 & & 2.077 \\
\hline 440 & 4016647 & 22 & & 2.072 & & 1.429 & 10. & 1.73 & 3.673 & 0.651 & 18 & 0.018 & & 2.965 \\
\hline 441 & .5137195 & 375 & & 0.249 & & 0.356 & & 0.795 & 27 & 1.114 & & 0.001 & & 0.234 \\
\hline 442 & 3191453 & 30.7913209 & & 0.298 & & 0.016 & 0. & 0.462 & 1. & 0.687 & 2. & 0.159 & 9.0 & -99.0 \\
\hline 443 & 23.6645764 & 30.800065 & -99.0 & -99.0 & 0 & 296 & 0.2 & 0.284 & 0.72 & 0.355 & 1.789 & 0.089 & -99.0 & -99.0 \\
\hline 444 & 23.5321019 & 30.8054253 & 0. & 2.045 & & 2.109 & 0. & 1.232 & 1.485 & 0.887 & 1.0 & 0.003 & 99.0 & -99.0 \\
\hline 445 & 23.6687348 & 30.8042817 & -99.0 & -99.0 & -99.0 & -99.0 & 1.271 & 2.227 & 0.82 & 1.426 & 0.98 & 0.25 & .706 & 0.584 \\
\hline 446 & 23.4427185 & 30.8706901 & 02 & 0.447 & 0.1 & 0.466 & 0. & 0.665 & 3.18 & 0.59 & 6.94 & 0.024 & 5.24 & 0.255 \\
\hline 447 & .5480965 & 31.0422525 & -99.0 & -99.0 & -99.0 & -99.0 & 0.073 & 0.709 & 0.224 & 0.287 & 0. & 0.001 & -99.0 & -99.0 \\
\hline 448 & 23.4258971 & 30.7960688 & 0.216 & 1.368 & 0.178 & 1.406 & 1.129 & 0.932 & 2.912 & 0.933 & 4.284 & 0.124 & 99.0 & -99.0 \\
\hline 449 & 4382839 & 84 & -99.0 & -99.0 & -96 & -99.0 & & 2.703 & -95 & -99.0 & & 0.059 & 0 & -99.0 \\
\hline 450 & 23.5158707 & 30.9160971 & 0 & 1.767 & 0.37 & 1.863 & & 1.426 & 17.05 & 1.468 & 1 & 0.053 & 27.429 & 1.73 \\
\hline 451 & 23.3907915 & 30.78 & & 1.068 & 0.11 & 0.819 & & 0.653 & 8.5 & 0.686 & & 0.002 & & 1.579 \\
\hline 452 & 827 & & -99.0 & -99.0 & -99.0 & -99.0 & & 1.353 & 12.783 & 0.828 & 1.02 & 0.071 & & -99.0 \\
\hline 453 & 945 & & & 2.792 & -99.0 & -99.0 & & 1.566 & -99.0 & -99.0 & & 0.156 & 99.0 & -99.0 \\
\hline 454 & 23.59 & & & 2.004 & & 1.713 & & 0.941 & & 0.994 & & 0.046 & & 1.38 \\
\hline 455 & 23.569164 & 30.78 & & 2. & & 2.924 & 10.95 & 0.431 & 1.855 & 0.913 & 6.25 & 0.231 & -99.0 & -99.0 \\
\hline 456 & 23.4667165 & 30.78 & & 2.537 & & 2.611 & 0. & 1.829 & 3.2 & 1.916 & 1.44 & 0.019 & 8. & 0.34 \\
\hline 45 & 23.5455554 & 30.7764174 & -99.0 & -99.0 & & 951 & 1.5 & 1.489 & 12.02 & 1.761 & 6.62 & 0.019 & 8.298 & 0.105 \\
\hline 458 & 23.5743777 & 30.8800705 & & 0.118 & & 0.479 & 0.63 & 0.553 & 4.65 & 0.582 & 4.377 & 0.026 & 0.9 & 0.275 \\
\hline 459 & 23.4482496 & 30.9017202 & -99.0 & -99.0 & -99.0 & -99.0 & 0.2 & 1.414 & 2.1 & 1.185 & 0.52 & 0.002 & 2.63 & 1.226 \\
\hline 460 & 23.3412458 & 30.7959015 & 0.38 & 2.063 & 0.3 & 1.901 & 2.34 & 2.148 & -99.0 & -99.0 & 6.75 & 0.021 & 11.23 & 1.242 \\
\hline
\end{tabular}


Table 3. continued.

\begin{tabular}{|c|c|c|c|c|c|c|c|c|c|c|c|c|c|c|}
\hline \multirow[t]{2}{*}{ Num. } & \multirow{2}{*}{$\begin{array}{c}\text { RA (J2000) } \\
\left({ }^{\circ}\right)\end{array}$} & \multirow{2}{*}{$\begin{array}{c}\text { Dec (J2000) } \\
\left({ }^{\circ}\right)\end{array}$} & \multicolumn{2}{|r|}{$3.6 \mu \mathrm{m}$} & \multicolumn{2}{|r|}{$4.5 \mu \mathrm{m}$} & \multicolumn{2}{|c|}{$\overline{5.8 \mu \mathrm{m}}$} & \multicolumn{2}{|r|}{$8.0 \mu \mathrm{m}$} & \multicolumn{2}{|r|}{$24 \mu \mathrm{m}$} & \multicolumn{2}{|r|}{$\overline{\mathrm{H} \alpha}$} \\
\hline & & & $F_{v}(\mathrm{mJy})$ & $\delta r$ & $F_{v}(\mathrm{mJy})$ & $\delta r$ & $F_{v}(\mathrm{mJy})$ & $\delta r$ & $F_{v}(\mathrm{mJy})$ & $\delta r$ & $F_{v}(\mathrm{mJy})$ & $\delta r$ & $F\left(10^{-14} \mathrm{erg} \mathrm{s}^{-1}\right)$ & $\delta r$ \\
\hline 461 & 23.5113866 & 30.8281243 & 0.47 & 2.435 & 0.14 & 0.677 & 0.086 & 0.472 & 2.55 & 2.504 & 1.29 & 0.055 & 0.253 & 2.035 \\
\hline 462 & 23.5086251 & 30.8769465 & 21 & 0.566 & 0.1 & 485 & & 0.476 & 2.84 & 0.514 & 69 & 0.081 & -99.0 & -99.0 \\
\hline 463 & 23.3723671 & 30.8372253 & 0.499 & 0.765 & 7 & 1.38 & 0.09 & 1.641 & 84 & 1.135 & 0.42 & 0.116 & 0.8 & 1.02 \\
\hline 464 & .6725383 & 30.78 & 0.22 & 2.399 & -9 & -99.0 & 4.71 & 2.752 & 21.68 & 2.751 & 34 & 0.0 & -99.0 & -99.0 \\
\hline 465 & 3.6007448 & 30.913 & 0.3 & 1.951 & 16 & 2.19 & 0.32 & 0.695 & 2.12 & 0.316 & .74 & 0.045 & 12.847 & 1.013 \\
\hline 466 & 3.6532015 & 30.8682463 & 25 & 2.433 & 17 & 1.909 & -99.0 & -99.0 & 0.27 & 0.712 & 32 & 0.112 & -99.0 & -99.0 \\
\hline 467 & 23.4657954 & 30.8688818 & 0.159 & 0.468 & 0.1 & 0.524 & 0.102 & 0.57 & 0.44 & 0.523 & 71 & 0.001 & -99.0 & -99.0 \\
\hline 468 & 23.4579162 & 30.8824059 & -99.0 & -99.0 & -99.0 & -99.0 & .56 & 0.874 & 1.183 & 1.022 & 1.351 & 0.17 & 2.424 & 0.128 \\
\hline 469 & 23.511233 & 30.8969572 & 0.28 & 1.414 & 0.17 & 1.425 & 0.13 & 0.558 & 0.977 & 0.64 & 1.15 & 0.001 & 0.044 & 2.637 \\
\hline 470 & 23.2262503 & 30.8403702 & 0.04 & 1.501 & 0.047 & 1.448 & 0.242 & 1.217 & 0.83 & 0.583 & .84 & 0.002 & 6.447 & 1.539 \\
\hline 471 & 23.5508254 & 30.8873415 & 1.84 & 0.428 & 1.17 & 0.51 & 0.86 & 0.559 & 0.73 & 0.564 & 0.48 & 0.085 & -99.0 & -99.0 \\
\hline 472 & 23.5687729 & 30.8724925 & 0.69 & 2.845 & 0.255 & 0.602 & 2.77 & 0.832 & 6.56 & 0.745 & 36.11 & 0.031 & -99.0 & -99.0 \\
\hline 473 & 23.6242498 & 30.9545948 & 68 & 2.537 & 0.87 & 2.502 & 6.21 & 1.865 & 42.24 & 0.942 & 67.42 & 0.064 & 92.56 & 1.218 \\
\hline 474 & 23.5446277 & 31.0 & 14 & 1.366 & .03 & 0.722 & 0.21 & 1.143 & 1.53 & 1.486 & 1.89 & 0.002 & 1.92 & 1.437 \\
\hline 475 & 3.6807839 & 74 & -99.0 & -99.0 & 0.05 & 0.144 & 0.111 & 0.406 & 0.252 & 0.398 & 13 & 0.003 & 0.074 & 2.947 \\
\hline 476 & .5 & 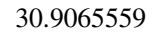 & 17 & 1.073 & & 1.382 & & 0.623 & 1 & 0.495 & 87 & 0.002 & 5.4 & 0.669 \\
\hline 477 & 23.5 & 2 & 0.032 & 1.334 & 02 & 1.396 & 0.15 & 1.37 & $\mathrm{~J}$ & 1.225 & 9 & 0.156 & -99.0 & -99.0 \\
\hline 478 & 75 & & & 1.995 & 0.41 & 0.839 & 0 & 0.788 & & 0.588 & 17.38 & 0.007 & -99.0 & -99.0 \\
\hline 479 & 14 & & & 1.224 & 13 & 1.228 & .73 & 1. & 8 & 1.415 & 38 & 0.0 & 161.77 & 1.006 \\
\hline 480 & 23.4 & 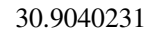 & 0. & 2.531 & 0. & 1.812 & 11 & 0. & 0.966 & 0.08 & 0 & 0.066 & 58 & 1.259 \\
\hline 481 & 23.54 & 7 & -99.0 & -99.0 & 0.033 & 0.786 & 0.106 & 0.639 & 0.11 & 0.789 & 21 & 0.156 & -99.0 & -99.0 \\
\hline 482 & 23.45 & 3 & -99.0 & -99.0 & 0.37 & 1.38 & 1.21 & 1.655 & 4.08 & 1.438 & 3.79 & 0.001 & -99.0 & -99.0 \\
\hline 483 & 3.5129404 & 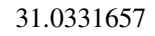 & 0.14 & 1.093 & 0.078 & 1.101 & 0.116 & 0.982 & 0.072 & 1.052 & 0.31 & 0.124 & -99.0 & -99.0 \\
\hline 484 & 23.5609306 & .8959397 & 0.79 & 1.157 & 0.34 & 1.324 & 0.8 & 1.116 & 4.38 & 1.324 & 38 & 0.002 & 2.27 & 2.53 \\
\hline 485 & 23.4593204 & 30.97 & 0.044 & 1.653 & 0.08 & 0.654 & 0.18 & 0.8 & 0.58 & 0.804 & .21 & 0.001 & -99.0 & -99.0 \\
\hline 486 & 3.5148818 & 39 & -99.0 & -99.0 & 0.48 & 2.696 & 4.99 & 0.859 & 3.182 & 0.985 & 1.9 & 0.023 & -99.0 & -99.0 \\
\hline 487 & 23.5609434 & 30.7 & 0.251 & 0.868 & 0.268 & 0.89 & 0.317 & 0.985 & 0.334 & 1.118 & 4.875 & 2.229 & -99.0 & -99.0 \\
\hline 488 & 3.6074669 & 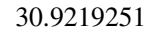 & & 0.319 & 1.93 & 0.377 & 20 & 0.377 & 2.99 & 0.429 & 26 & .001 & -99.0 & -99.0 \\
\hline 489 & 3.5 & 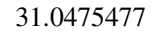 & & 0.457 & & .753 & 097 & 0.691 & & 0.804 & 64 & .002 & -99.0 & -99.0 \\
\hline 490 & 3 & & & & & 0.881 & & 91 & & 1.083 & & 0.018 & -99.0 & -99.0 \\
\hline 491 & 8 & & & 0. & & 0.662 & & & & 19 & & 0.033 & 2.45 & 0.303 \\
\hline 492 & 6 & & 9.0 & -99.0 & 0 & -99.0 & -99.0 & -99.0 & -99.0 & -99.0 & 4 & 0.072 & -99.0 & -99.0 \\
\hline 493 & 2 & & 0 & 0.308 & 0. & 0.762 & & 46 & & 1.141 & & 0.001 & 6.8 & 0.054 \\
\hline 494 & 6 & 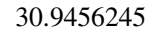 & & 1.2 & & 1.243 & -99.0 & -99.0 & -99.0 & -99.0 & 46.92 & 0.005 & 56.046 & 0.362 \\
\hline 495 & 25 & 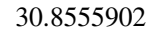 & & 1.781 & 0 & 2.335 & 0.54 & 2.765 & 1.07 & 1.708 & .74 & 0.001 & 1.09 & 1.571 \\
\hline 496 & 3.6 & 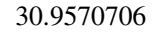 & & 0.891 & 0 & 0.764 & 46 & 0.521 & 3.469 & 0.395 & 5.024 & 0.091 & -99.0 & -99.0 \\
\hline 497 & 23.5328498 & 31.0 & 0.027 & 0.123 & $0.0 c$ & 0.874 & 0.191 & 0.794 & 0.17 & 0.754 & 32 & 0.001 & -99.0 & -99.0 \\
\hline 498 & 23.4575392 & 99 & & 0.95 & 3 & 0.806 & 33 & 0.831 & .73 & 0.753 & 31 & 0.003 & -99.0 & -99.0 \\
\hline 499 & 23.5926926 & 0.9 & 0. & 0.597 & 0.32 & 0.548 & 0.09 & 0.103 & 1.5 & 0.483 & 36 & 0.001 & -99.0 & -99.0 \\
\hline 500 & 23.340851 & 30.92 & 0 & 0.799 & 0.27 & 0.51 & -99.0 & -99.0 & 0.49 & 0.375 & 1.89 & 0.001 & -99.0 & -99.0 \\
\hline 501 & .5026165 & 01 & & 2.145 & 0 & 057 & 0.7 & 1.265 & 2.43 & 1.272 & 0 & 0.0 & -99.0 & -99.0 \\
\hline 502 & 23.5112777 & 784 & -99.0 & -99.0 & -99.0 & -99.0 & -99.0 & -99.0 & 600 & 1.368 & 59 & 0.07 & 0.212 & 2.052 \\
\hline 503 & 23.51 & 64 & 0.212 & 0.391 & 187 & 461 & 115 & 0.406 & & 3 & 448 & 0.033 & .363 & 0.523 \\
\hline 504 & 98 & & & 1.977 & 0 & 0.721 & 0.3 & 0.594 & 1.308 & 1.555 & 68 & 0.124 & -99.0 & -99.0 \\
\hline 505 & 24 & 3 & & 0.76 & & 0.831 & & 0.8 & & 0.801 & 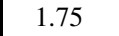 & 0.002 & 0.56 & 2.891 \\
\hline 506 & 23.4 & 30 & 0.012 & 0.911 & 0.023 & 0.845 & 0.174 & 0.842 & & 0.622 & 0.2 & 0.147 & -99.0 & -99.0 \\
\hline 507 & 23.5 & & & 1.315 & & 1.214 & & 1.729 & & & 93 & 194 & 31.03 & 1.072 \\
\hline 508 & & 30 & & 0.9 & 0 & & & & & & & & -99.0 & -99.0 \\
\hline 509 & 23.4 & & & 0.852 & & 65 & & 1.013 & & 0.779 & & 0.089 & -99.0 & -99.0 \\
\hline 510 & 23.31 & 30.8 & & 1.476 & 0.112 & & & 0.919 & & $1 .($ & & .352 & 8.289 & 0.738 \\
\hline 511 & 23.58 & & -99.0 & -99.0 & & 0.297 & 16.07 & 0.502 & 3.436 & 1.159 & 23.32 & 0.046 & 45.95 & 0.643 \\
\hline 512 & 23.50917 & 30.8 & & 1.973 & & & & 0. & 7.34 & 1.051 & 89 & 0.0 & 5.11 & 0.429 \\
\hline 513 & 23.3850146 & 31.0214745 & & & & 0.832 & 0.178 & 0.505 & (1) & 0.663 & 23 & 0.001 & -99.0 & -99.0 \\
\hline 514 & & & & -99.0 & - & -99.0 & & 2.8 & & 2.027 & 99 & .065 & 14.55 & 1.43 \\
\hline 515 & 23.5390177 & 30.9218105 & 0.379 & 0.419 & 0.483 & 0.405 & 0.56 & 0.406 & 0.79 & 0.506 & 0.35 & 0.001 & -99.0 & -99.0 \\
\hline
\end{tabular}

